
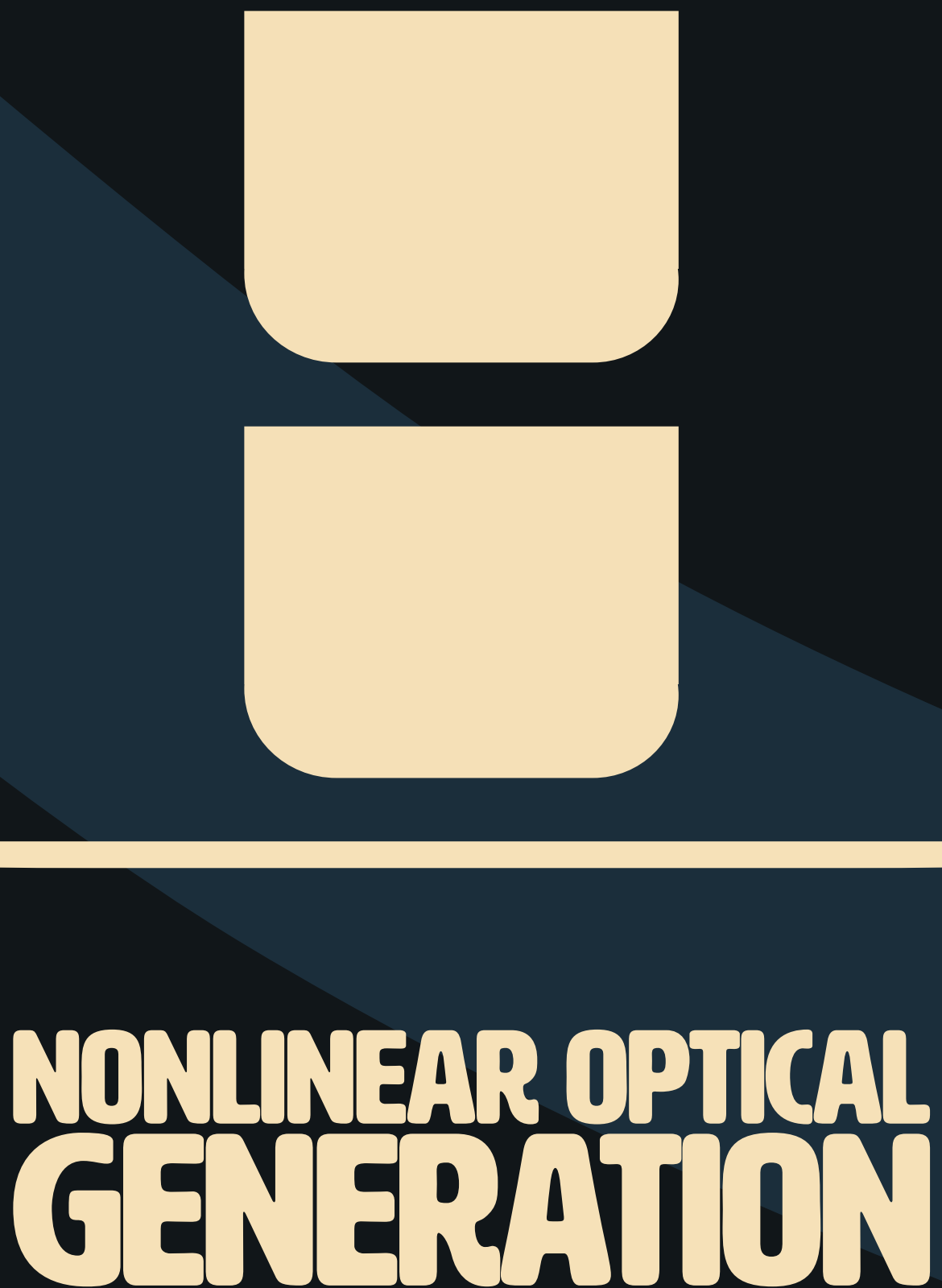

AND ACOUSTO-OPICAL CONIRAL OF LGHI

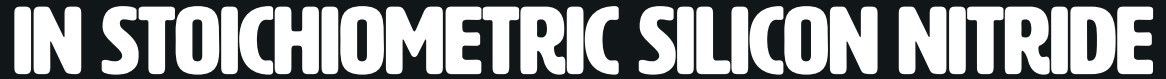
TIIECRAIED WAIECIIIS

Marco Antonio Garcia Porcel 


\section{Nonlinear optical generation and acousto-optical control of light in stoichiometric silicon nitride integrated waveguides}




\section{Graduation committee:}

\section{Chairman \& secretary:}

Prof. dr. ir. A. Brinkman

\section{Supervisor:}

Prof. dr. K.-J. Boller

\section{Co-supervisor:}

Dr. P.J.M. van der Slot

\section{Members}

Prof. dr. C. Becher

Prof. dr. J.L. Herek

Prof. dr. ir. G. Krijnen

Prof. dr. M. Schmidt
University of Twente

University of Twente

University of Twente

Universität des Saarlandes

University of Twente

University of Twente

Friedrich-Schiller Universität Jena

Cover design: Set of three, by Anouk, Chris, Marco and Eliana

Publisher: Marco Antonio Garcia Porcel

No part of this work may be reproduced by print, photocopy or any other means without the writen permission from the publisher.

ISBN: 978-90-365-4435-1

DOI: $10.3990 / 1.9789036544351$ 


\title{
Nonlinear optical generation and acousto-optical control of light in stoichiometric silicon nitride integrated waveguides
}

\author{
DISSERTATION
}

to obtain

the degree of doctor at the University of Twente, on the authority of the rector magnificus, prof. dr. T.T.M. Palstra, on account of the decision of the graduation committee, to be publicly defended on Friday the $8^{\text {th }}$ December 2017 at 16:45

by Marco Antonio Garcia Porcel

born on the 1st of February 1986 in Guadix (Granada), Spain 
This dissertation has been approved by:

Supervisor: Prof. dr. K.-J. Boller

Co-supervisor: Dr. P.J.M. van der Slot
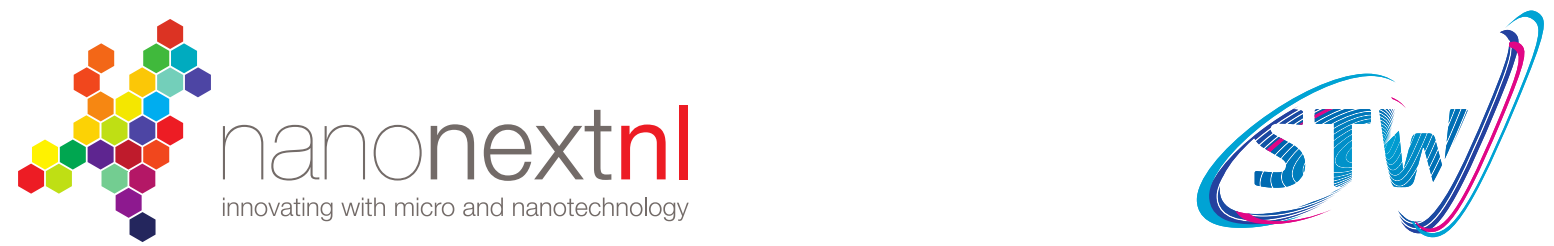

The research presented in this thesis was carried out at the Laser Physics and Nonlinear Optics group, MESA+ Institute for Nanotechnology, Department of Science and Technology, University ot Twente, The Netherlands. This thesis is part of NanoNextNL, a micro and nanotechnology innovation consortium of the Government of the Netherlands and 130 partners from academia and industry. More information on www.nanonextnl.nl. This research is also supported in part by the Dutch Technology Foundation STW, which is part of the Netherlands Organization for Scientific Research NWO. 


\section{Contents}

Summary ix

Nederlandse Samenvatting $\quad$ xiii

1 Introduction 1

2 Theory: nonlinear optics in amorphous waveguides 11

2.1 Waveguide optics . . . . . . . . . . . . . . . . . . . . 12

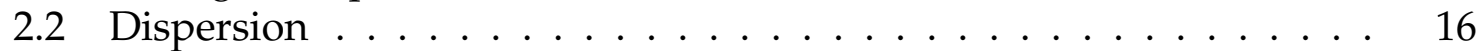

2.3 Nonlinear optical polarization . . . . . . . . . . . . . . . . 20

2.4 Phase matching . . . . . . . . . . . . . . . . . . . 22

2.5 Nonlinear optics in amorphous materials . . . . . . . . . . . . . . 24

2.6 Coherent photogalvanic effect . . . . . . . . . . . . . 27

2.7 Acousto-optic interaction:

Surface acoustic waves . . . . . . . . . . . . . . . . . . . . . . . 29

3 Supercontinuum generation $\quad 35$

3.1 Introduction . . . . . . . . . . . . . . . 36

3.2 Experimental Setup . . . . . . . . . . . . . . . . . . . 37

3.3 Experimental Results . . . . . . . . . . . . . . . . . . 40

3.4 Numerical Simulations . . . . . . . . . . . . . . . . . . . . . . . 42

3.5 Dependence on Waveguide Width . . . . . . . . . . . . . . 45

3.6 Summary and Conclusions ..................... 45

4 Refractive index modulation via surface acoustic waves 53

4.1 Introduction ........................ 54

4.2 Acousto-optic refractive index modulation . . . . . . . . . . . 56

4.3 Geometry and simulation domain . . . . . . . . . . . . 58

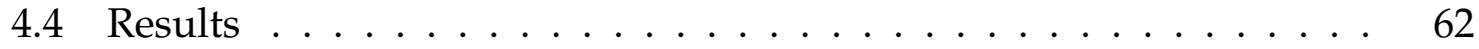

4.4.1 Acoustic wave generation ..................... 62

4.4.2 Modulation of the effective refractive index . . . . . . . . . . . 66

4.5 Summary and Conclusions . . . . . . . . . . . . 71

5 Second-harmonic generation $\quad 77$ 


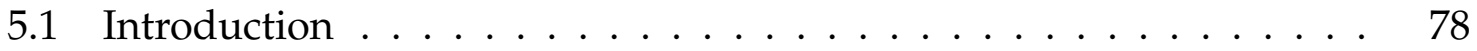

5.2 Experimental procedure and results $\ldots \ldots \ldots \ldots \ldots \ldots$

5.3 Discussion . . . . . . . . . . . . . . . . . 87

5.3.1 Summary and conclusions . . . . . . . . . . . . . 94

6 Conclusion and outlook $\quad 99$

$\begin{array}{ll}\text { Acknowledgements } & 107\end{array}$ 
A Eli y a mi familia. 



\section{Summary}

Integrated photonics is a key enabling technology to address various industrial and societal issues. In particular, integrated photonics plays an important role in fulfilling the ever-increasing demand for higher speed and larger bandwidth created by our information-based society. The large variety of required optical functionalities has led to a range of integrated photonic platforms, each optimized to manipulate and control light in a particular way. Extending the capabilities of each of the platforms is paramount in overcoming current limitations of these platforms. In this thesis, we consider light propagating in one of these integrated photonic platforms that consists of a stoichiometric silicon nitride $\left(\mathrm{Si}_{3} \mathrm{~N}_{4}\right)$ core surrounded by a silicon oxide cladding, the so-called TriPleX ${ }^{\mathrm{TM}}$ platform. Silicon nitride waveguides have the advantage of a large transparency range, from the visible to the mid-infrared, the lowest propagation loss of all integrated photonics waveguide platforms, a large bandgap energy, a moderate nonlinear response, while the manufacturing process is also compatible with CMOS fabrication. Furthermore, silicon nitride waveguides can be tapered to allow hybrid integration with low coupling losses. Enhancing the control over and manipulation of light propagating through these waveguides would significantly enhance the optical functionalities that can be implemented using this platform and thus increase its attractiveness.

In this thesis, we have studied three different nonlinear optical approaches to manipulate light propagating through stoichiometric silicon nitride waveguides. In particular, we have used nonlinear optical processes for extreme spectral broadening of a short pulse injected into a properly prepared waveguide, for phase and amplitude modulation of the injected light and for conversion to a single, secondharmonic wavelength.

In Chapter 2, we provide the theoretical background for linear and nonlinear propagation of light through amorphous dielectric waveguides. The description of linear light propagation includes the concepts of eigenmodes and dispersion, while the nonlinear light propagation includes phase matching and various nonlinear processes associated with the different orders of the nonlinear material response. These include, second-harmonic generation and supercontinuum generation, which is the result of a combination of third-order nonlinear processes. Chapter 2 concludes with a brief description of the coherent photogalvanic effect and of surface acoustic waves. 
In Chapter 3, we show our results on supercontinuum generation in low-loss, dispersionengineered $\mathrm{Si}_{3} \mathrm{~N}_{4}$ waveguides using a commercial pulsed laser working at a wavelength of $1560 \mathrm{~nm}$ (C-band for optical communications) and having a pulse duration of $120 \mathrm{fs}$ as pump laser. We have realized a bandwidth of $454 \mathrm{THz}$ with a supercontinuum spectrum that extends well into the mid-infrared. This result is compared with the theoretical prediction obtained using a one-dimensional generalized nonlinear Schroedinger equation. The simulated supercontinuum spectra is in excellent agreement with the measured spectrum except that the required waveguide-internal pulse energy was a factor of 3.6 lower in simulation compared to the experiment. We attribute this difference to a lower nonlinear refractive index of the waveguides we used, however further study is required to identify the source of the observed discrepancy. In addition, both, the experiments and the simulations, show that for increasing the width of the core the generated spectrum is shifted towards longer wavelengths by about $2.45 \mathrm{~nm}$ per $\mathrm{nm}$ width.

While Chapter 3 focuses on broadening the spectral content of light when it propagates through the amorphous silicon nitride waveguide, we study in Chapter 4 how the phase and amplitude of the propagating light can be changed. The method describe in this chapter relies on the strain induced in the region of the optical mode propagating through a buried waveguide by a surface acoustic wave. The strain modulation induces a refractive index modulation, which translates into a modulation of the phase upon propagation of the light. When combined with a so-called Mach-Zehnder interferometer (MZI), this phase modulation can be converted to an amplitude modulation. The surface acoustic wave is generated in a thin piezoelectric layer placed on top of the cladding by a so-called interdigitated transducer (IDT). In order to determine optimum conditions for modulation of the phase of the light, we have numerically studied the dependence of the index modulation on the thickness of the piezo-electric film and period of the acoustic wave (i.e., period of the IDT electrode) and for four combinations of IDT placement and terminating layer. The best configuration was found for an IDT period of $30 \mu \mathrm{m}$ with a PZT thickness of $4 \mu \mathrm{m}$ with the IDT placed at the cladding-PZT interface and a conductive terminating layer at the PZT-air interface. For this configuration, a maximum relative refractive index change $\Delta n / n_{\text {eff }}=0.14 \%$ is found. If this maximum change in effective refractive index is combined with a MZI, where the two arms are separated by half an acoustic wavelength so that tensile and compressive strain doubles the phase difference that can be realized over a given length, a length of $103 \mu \mathrm{m}$ is sufficient to obtain full amplitude modulations of the guided light.

After studying spectral broadening in Chapter 3 and phase and amplitude modulation in Chapter 4 , we use in Chapter 5 the coherent photogalvanic process to create an effective second-order susceptibility grating in the amorphous, stoichiometric silicon nitride waveguide and use this grating to produce second-harmonic generation. The laser used for both the coherent photogalvanic effect as well as 
for second-harmonic generation is a fiber laser producing 6.2-ps optical pulses at a wavelength of $1064 \mathrm{~nm}$. A second-order nonlinear grating has been created and second-harmonic generation has been observed in waveguides with a height of $0.9 \mu \mathrm{m}$ and various widths for the core. The experimental observations show that the second-order nonlinear grating is written over a time period of minutes to several hours depending on the waveguide core dimensions and the average power of the pump laser. After the grating has been written, the second-harmonic light was instantaneously generated, at least within our measurement accuracy. With an established grating, we have verified that the average power of the second-harmonic radiation quadratically depends on the average power of the pump. The gratings turn out to be long-lived and hardly any degradation in the second-harmonic signal is observed after a shelf-time of a week in a dark environment. The measured polarization of the second-harmonic radiation is the same as that of the fundamental pump light. Rotation of the pump polarization by 90 degrees results in rewriting of a new effective second-order nonlinear grating that now matches the new polarization direction. A maximum conversion efficiency of $0.4 \%$ has been obtained using an average waveguide-internal pump power of $13 \mathrm{~mW}$. Assuming the second harmonic radiation is emitted in a single transverse mode, the measured conversion efficiency translates into an effective second-order susceptibility of $3.2 \mathrm{pm} / \mathrm{V}$.

The work presented in this thesis shows that nonlinear optical processes can be used to control to a greater extent the properties of light in stoichiometric silicon nitride waveguides. Making use of these processes enhances the functionalities available for this platform, which is promising for many different applications, for example, high precision metrology, broadband communications and optical quantum computing. 



\section{Nederlandse Samenvatting}

Geïntegreerde fotonica vervult een sleutelrol in het vinden van oplossingen voor een verscheidenheid aan industriële en maatschappelijke problemen. In het bijzonder speelt geïntegreerde fotonica een belangrijke rol in realiseren van steeds grotere bandbreedte en snellere informatieoverdracht om aan de eisen van onze informatiemaatschappij te voldoen. De grote verscheidenheid aan optische functionaliteiten heeft geleid tot een scala van geïntegreerde fotonische platformen, waarbij elk platform is geoptimaliseerd voor bepaalde manipulatie en beheersing van het licht. Om huidige tekortkomingen te overbruggen is het van groot belang om voor elk van de platformen de mogelijkheden om licht te manipuleren uit te breiden. In dit proefschrift beschouwen we licht dat zich voortplant in een van deze platformen. Dit platform is gebaseerd op een stoichiometrische silicium nitride $\left(\mathrm{Si}_{3} \mathrm{~N}_{4}\right)$ kern, welke omgeven is door silicium oxide: het zogeheten TriPleX ${ }^{\mathrm{TM}}$ platform. Silicium nitride golfgeleiders hebben een aantal grote voordelen, waaronder een groot transparantiegebied (van zichtbaar licht tot het midden-infrarood), het laagste voortplantingsverlies van alle geïntegreerde fotonische golfgeleiders, een grote bandkloof, en een gematigde niet-lineaire respons. Bovendien is het fabricageproces compatibel met CMOS fabricage. Verder kan de vorm van een silicium nitride golfgeleider veranderd worden ten behoeve van hybride integratie met zeer lage koppelingsverliezen. De implementatie van optische functionaliteiten kan significant verbeterd worden indien de controle over en de manipulatie van het licht in deze golfgeleider uitgebreid wordt, met alle bijbehorende voordelen voor toepassingen.

In dit proefschrift bestuderen we drie verschillende niet-lineaire optische methoden om licht te manipuleren in stoichiometrische silicium nitride golfgeleiders. Hierbij is in het bijzonder gekeken naar niet-lineaire optische processen voor extreme verbreding van het spectrum van een korte puls, welke geïnjecteerd wordt in de golfleider, voor fase- en amplitudemodulatie van het geïnjecteerde licht en voor het converteren van de golflengte van de pomppuls naar de tweede harmonische golflengte.

In hoofdstuk 2 behandelen we de theoretische achtergronden voor lineaire en nietlineaire voortplanting van licht door amorfe diëlektrische golfgeleiders. De beschrijving van de lineaire voortplanting van licht behelst de concepten van eigenmodi en dispersie, terwijl de beschrijving van niet-lineaire voortplanting het concept van 
overeenkomende fases en verschillende niet-lineaire processen, behorende bij de verschillende ordes van de niet-lineaire materiaalresponsie, omvat. Hieronder valt tweede-harmonische generatie en supercontiuüm generatie, wat het resultaat is van een combinatie van derde-orde niet-lineaire processen. Hoofdstuk 2 eindigt met een korte beschrijving van het coherente fotogalvanische effect en akoestische oppervlaktegolven.

In hoofdstuk 3 tonen we onze resultaten met betrekking tot supercontinuüm (SC) generatie in $\mathrm{Si}_{3} \mathrm{~N}_{4}$ golfgeleiders met lage verliezen en speciaal ontworpen dispersie welke gepompt worden door een commercieel ver-krijg-ba-re gepulste laser werkend op een golflengte van $1560 \mathrm{~nm}$ (C-band voor optische communicatie) en met een pulslengte van $120 \mathrm{fs}$. Hierbij bereiken we een bandbreedte van $454 \mathrm{THz}$ met een SC spectrum dat zich uitstrekt tot ver in het mid-infrarood. Dit resultaat wordt vergeleken met de theoretische voorspelling op basis van de eendimensionale gegeneraliseerde Schrödingervergelijking. Het gesimuleerde supercontinuüm spectrum komt uitstekend overeen met het gemeten spectrum, met als kanttekening dat de benodigde pulsenergie in de golfgeleider een factor 3.6 lager was dan in het experiment. We wijten deze discrepantie aan een lagere niet-lineaire brekingsindex van de golfgeleiders in het experiment. Meer onderzoek is echter nodig om de bron van dit verschil definitief te achterhalen. Zowel de experimenten als simulaties tonen aan dat met het vergroten van de breedte van de kern van de golfgeleider het gegenereerde spectrum verschuift naar lagere golflengtes met ongeveer $2.45 \mathrm{~nm}$ per nm breedte.

Terwijl hoofstuk 3 zich richt op het verbreden van de spectrale inhoud van het licht tijdens propagatie door de amorfe silicium nitride golfgeleider, bestuderen we in hoofdstuk 4 hoe de fase en amplitude van het propagerende licht veranderd kan worden. De methode die wordt beschreven in dit hoofdstuk is gebaseerd op de mechanische vervorming in het materiaal waar de optische mode zich bevindt en welke wordt gegenereerd door een akoestische oppervlaktegolf. De door de akoestische golf ver-oor-zaak-te modulatie in de vervorming heeft een modulatie van de brekingsindex als gevolg, wat zich vertaald in een modulatie in de fase van het licht. Als een Mach-Zehnder interferometer (MZI) wordt gebruikt kan deze fasemodulatie worden omgezet in een amplitudemodulatie. De akoestische oppervlaktegolf wordt gegenereerd door een zogenaamde geïnterdigiteerde transducer (IDT) in een dunne piëzo-elektrische film welke geplaatst is op de waveguide. Om de optimale condities voor modulatie van de fase van het licht te bepalen, hebben we een aantal numerieke simulaties uit-ge-voerd om te onderzoeken hoe de modulatie van de brekingsindex afhangt van de dikte van de piëzo-elektrische film, de periode van de akoestische golf (d.w.z., de periode van de IDT), en voor vier combinaties van plaatsing van de IDT en een afsluitende film. De beste configuratie bleek een IDT periode van $30 \mu \mathrm{m}$ te zijn, met een PZT laagdikte van $4 \mu \mathrm{m}$. Dit zorgt voor een verandering van de brekingsindex van ongeveer $\Delta n / n_{\text {eff }}=0.14 \%$. Als deze 
maximum verandering van de effectieve brekingsindex zou worden gecombineerd met een MZI, waarbij de afstand tussen de armen gelijk is aan een halve akoestische golflengte zodat de trek- en compressievervorming het faseverschil verdubbelt over een gegeven lengte, is een lengte van $103 \mu \mathrm{m}$ genoeg om volledige amplitude modulatie van het licht te bereiken.

$\mathrm{Na}$ het bestuderen van spectrale verbreding in hoofdstuk 3 en fase- en amplitudemodulatie in hoofdstuk 4, gebruiken we in hoofdstuk 5 het coherente fotogalvanisch proces om een effectief tweede-orde susceptibiliteitrooster in een amorfe, silicium nitride golfgeleider te creëren. Vervolgens wordt dit rooster gebruikt voor tweedeharmonische generatie van de pump puls. De laser die we gebruiken voor zowel het coherente galvanische effect als tweede-harmonische generatie is een fiber laser welke 6.2-ps optische pulsen genereert met een golflengte van $1064 \mathrm{~nm}$. Tweedeorde niet-lineair roosters zijn succesvol gemaakt in golfgeleiders met een hoogte van $0.9 \mu \mathrm{m}$ en voor verschillende breedtes van de kern. In deze golfgeleiders is ook succesvol tweede-harmonische generatie waargenomen. De experimentele waarnemingen tonen aan dat het tweede-orde niet-lineaire rooster over een tijdsperiode van minuten tot een paar uur geschreven wordt, afhankelijk van de afmetingen van de kern in de golfgeleider en het gemiddelde vermogen van de pomplaser. De roosters blijken langdurig stand te houden en er vindt nauwelijks degradatie van het tweede-harmonische signaal plaats na plaatsing in een donkere omgeving voor een week. De gemeten polarisatie van de tweede-harmonische straling is hetzelfde als van het fundamentele pomplicht. Een rotatie van de polarisatie van het pomplicht met 90 graden resulteert in het herschrijven van een nieuw effectief tweede-orde niet-lineair rooster dat met de nieuwe polarisatierichting overeenkomt. Een maximale conversierendement van $0.4 \%$ is gevonden voor een gemiddelde pompvermogen van $13 \mathrm{~mW}$ in de golfgeleider. Wanneer er wordt uitgegaan van emissie van de tweede-harmonische straling in een enkele transversale mode, dan volgt uit het conversierendement een effectieve, tweede-orde susceptibiliteit van $3.2 \mathrm{pm} / \mathrm{V}$.

De resultaten in dit proefschrift tonen aan dat niet-lineaire optische processen gebruikt kunnen worden om de controle over de eigenschappen van licht in stoichiometrische silicium nitride golfpijpen uit te breiden. Door deze processen te gebruiken komen voor dit platform meer optische functies beschikbaar, wat veelbelovend is voor een grote verscheidenheid aan applicaties zoals bijvoorbeeld zeer nauwkeurige metrologie, breedbandcommunicatie, en optische kwantumberekeningen. 

Chapter 1

\section{Introduction}


Making use of light enables countless scientific, industrial and societal innovation. This field, often called photonics, is considered a key enabling technology in our modern society [1,2]. For instance, the optical fiber [3] and the laser [4,5] are at the foundation of modern, high speed telecommunication and information technology [6,7]. Although there are many applications based on optical fibers, especially in long-distance data communication and various sensors, the sensitivity of fibers to their environment, specifically temperature changes and the low mechanical stability, limits applications to fully exploit the benefits of guided wave propagation. A different approach, with much higher intrinsic immunity to perturbations, is to implement the generation, control and detection of light into integrated optical waveguide circuits on chips, commonly referred to as photonic integrated circuits (PICs). These benefits and the option for small-size devices and mass fabrication have created a strong interest in integrated optics, first based on planar waveguides (one-dimensional integrated waveguides) $[8,9]$, later on two-dimensional or channel waveguides [8,9] and also on three-dimensional approaches [10].

State-of-the-art PICs show many functional applications in fields as different as metrology [11, 12], chemical sensing [13] or biology [14]. For instance, reduced production costs and recent research on microfluidics and lab-on-chip concepts have strongly stimulated the realization of advanced optical sensors for pharmaceutical, medical and environmental applications [13-15]. However, the most thriving PICs applications are expected to enable innovations in the telecommunication and information technology [11, 16-18], driven by a continuously growing demand for speed and bandwidth as well as the need to reduce the power consumption of current electronic solutions.

As the on-chip functionalities for telecom devices will become extremely complex, e.g., in the case of integrated microwave photonics (I-MWP) [18, 19], it is generally expected that all the required photonic components cannot be realized within a single waveguide platform. For instance, some semiconductor waveguide platforms allow the generation, modulation and detection of light, but only at the expense of relatively high propagation losses. Platforms based on dielectric materials provide low-propagation loss, which is essential for high resolution filters, optical delay lines and low noise performance, but the use of dielectrics for generating and modulating light is considered as limited.

Hybrid integration, i.e., combining semiconductor and dielectric building blocks, can be employed to enhance the functionality or performance of single waveguide platforms in many situations, such as the reduction of the spectral bandwidth of onchip lasers $[20,21]$ or for realizing chip-sized optical beam forming networks [22]. However, in order to exploit fully the potential of PICs and fulfill the expectations for telecommunications and other areas of application, hybrid integration alone is 
not sufficient, such as for frequency comb generation. Here, exploring on-chip nonlinear optical processes in dielectric photonic platforms can offer further options for the manipulation and tailoring of light. More specifically, nonlinear optics, especially with dielectric optical waveguides, will allow an increase in the extent of spectral and temporal control of light generation and guiding in chips. The most prominent examples are supercontinuum generation [23], as for generating phasecoherent frequency combs [24], using second-order nonlinearities for encoding information on light fields via electro-optic modulation [25] or cascading nonlinearities [26], and acousto-optic effects to achieve modulation in passive materials [27].

Implementing nonlinear optics in an integrated platform, first of all, is subjected to fundamental physical requirements. Due to the nature of the interaction of light with matter, nonlinear optical processes are intrinsically weak [25, 28, 29]. Therefore, such processes typically require increased light intensities over extended interaction lengths. These conditions can be realized employing guided light pulses at moderate average power using waveguides with a high optical confinement. For example, using waveguides with a high index contrast and suitably sized core area and cross-section, enables high light intensities, while the low loss in dielectric material enables extended propagation lengths. On the other hand, the high intensities used in nonlinear optical processes can also induce undesired nonlinear losses [25], specifically, two-photon absorption, which can be avoided by using materials with a spectrally wide transparency range, that is, with a bandgap energy at least twice the maximum photon energy of the light involved in the nonlinear processes.

A second condition required for efficient nonlinear optical processes, next to high light intensities and maximized nonlinearity is that, for the various different wavelengths involved, the light needs to encounter the appropriate dispersion for phase matching. In integrated optical waveguides, the wave dispersion can be controlled by the choice of the core and cladding materials, the geometry of the waveguide and the optical modes and polarization used [30,31]. The phase matching can further be controlled via so-called quasi-phase matching, i.e., if it is possible to provide a periodic structuring of the waveguide material's nonlinear susceptibility [25, 32].

As an intermediate summary, realizing efficient nonlinear optical processes in integrated photonics requires fulfilling a number of physical requirements. However, for a successful introduction of a technology that serves applications, these are not the only conditions. For example, using waveguide platforms that are compatible with CMOS [33] fabrication will tremendously benefit from the large, worldwide manufacturing base that allows large-volume production at low costs. Still, the large variety in applications for PICs, which is typical for an enabling technology, has driven the development of different platforms for optical integration. The prevailing technologies can be roughly divided into two categories. The first category can be described as not being compatible with CMOS fabrication [34] and 
includes platforms based on chalcogenides, aluminum oxide, indium phosphide or galium arsenide. The other category comprises platforms that are compatible with CMOS fabrication $[12,35,36]$, which includes material systems based on silicon-oninsulator (SOI), doped silica or silicon nitride $\left(\mathrm{SI}_{x} \mathrm{~N}_{y}\right.$, also briefly $\left.\mathrm{SiN}\right)$. In this work we will focus on a CMOS compatible material system and will therefore briefly compare the three, SOI, doped silica and SiN, materials with regard to their usefulness for nonlinear optical processes.

First, silicon-on-insulator is highly compatible with the standardized wavelengths used in modern telecommunication and has a relatively high nonlinearity. However, the relatively small bandgap results in a limited wavelength range, the near and mid infrared, due to two-photon absorption. This material also has a strong Raman response in the same range, which is usually undesired as well. The second material, doped silica $\left(\mathrm{SiO}_{2}\right)$ provides waveguides that have typically only a low confinement due to a small index contrast. On the other hand, the material has a much larger bandgap energy and consequently a large transparency range that reaches down to the visible, whereas the nonlinear response is rather weak. Consequently, long interaction lengths are required [32,37], making this platform less suitable for nonlinear optical processes. Finally, silicon nitride platforms have shown the lowest propagation loss for any integrated waveguide platform [38-41], and due to the large bandgap energy show an extremely large transparency range extending into the ultraviolet [42]. The large bandgap energy ensures that the nonlinear optical processes can extend over a large spectral range before nonlinear losses become noticeable. At the same time, the nonlinear response is larger than that of silica [4345]. Also, at a given input power, the intensities are higher due to much stronger confinement. Furthermore, the extremely low loss in combination with the high confinement via high index contrast, specifically as provided by stoichiometric silicon nitride when grown slowly, at high temperature via low-pressure chemical vapor deposition (LPVCD), allows for complex integrated photonic circuits, e.g., as being developed for integrated microwave photonics [18].

Considering these fundamental, technological and practical requirements, stoichiometric silicon nitride $\left(\mathrm{Si}_{3} \mathrm{~N}_{4}\right)$ was selected for this thesis as a most promising material for studying nonlinear optics in waveguides. Actually, the state-of-the-art of nonlinear optics in $\mathrm{Si}_{3} \mathrm{~N}_{4}$ PICs is already in rapid and exciting development. Thanks to newly developed fabrication strategies [46, 47] phase matching of thirdorder nonlinear processes has been demonstrated in $\mathrm{Si}_{3} \mathrm{~N}_{4}$. Nevertheless, harnessing the third-order nonlinearity further via dispersion engineering would allow, for instance, to develop on-chip coherent anti-Stokes Raman spectroscopy sources [48]. Another achievement is that supercontinuum generation has been successfully demonstrated at visible and near-infrared wavelength ranges [23] as well as frequency comb generation in the mid-infrared wavelength range [42, 49]. This material platform recently enabled the broadest ever on-chip supercontinuum generation having 
a bandwidth of almost $500 \mathrm{THz}$ [23]. One of the advantages of on-chip supercontinuum generation is that due to the higher nonlinearity typically shorter interaction lengths are required compared to, e.g., regular or photonic-crystal fibers, thereby potentially providing a more stable supercontinuum source. In this work, we demonstrated supercontinuum generation reaching for the first time from visible to midinfrared driven by a drive laser at telecommunication wavelengths.

The nonlinear optical processes mentioned so far for the $\mathrm{Si}_{3} \mathrm{~N}_{4}$ platform are all based on the third-order susceptibility. As an amorphous material, $\mathrm{Si}_{3} \mathrm{~N}_{4}$ is not expected to possess an intrinsic second-order susceptibility [25]. However, certain options can be considered that might allow breaking the symmetry in a waveguiding geometry, thereby enabling that also an effective second-order susceptibility might be exploit. In this work we demonstrate for the first time a second-order response in LPCVD-grown $\mathrm{Si}_{3} \mathrm{~N}_{4}$ waveguides. The effect that induces the response is known as the coherent photogalvanic effect and this has been previously observed, e.g., in silica glass fibers first by Margulis et al. [50].

So far, we have discussed controlling or generating light using the third and secondorder nonlinear susceptibility of the waveguide material. However, changes in a material susceptibility can be induced also via the interaction of light with other types of waves, such as acoustic waves, which induce changes in the first-order susceptibility, i.e., the linear refractive index. Such changes, in refractive index, control the phase of the guided light which can be used in combination with a so-called onchip Mach-Zehnder interferometer to create an intensity modulator [51, 52]. Various other physical processes can be used to control the linear refractive index in dielectrics [25] as well, such as thermally induced index changes or stress induced changes. However, these methods, especially thermal tuning, are much limited in modulation speed. In this thesis, we investigate via theoretical modeling the use of surface acoustic waves (SAWs) to modulate the effective linear refractive index of $\mathrm{Si}_{3} \mathrm{~N}_{4}$ waveguides to for Mach-Zehnder interferometer-based light modulator. In a more general sense, the named interaction with SAWs can be perceived as another nonlinear process as well, involving both optical and acoustic waves [25].

This thesis is organized as follows. In Chapter 2 we briefly present the relevant theory behind the various physical processes that play a role in manipulating the flow of light in $\mathrm{Si}_{3} \mathrm{~N}_{4}$ waveguides, in particular wave guiding and the processes that play a role in supercontinuum generation, Rayleigh type SAW and the coherent photogalvanic effect. In Chapter 3, we show that dispersion engineering can be used to obtain control over the supercontinuum spectrum pumped by a femtosecond Erdoped fiber laser operating at a telecommunication wavelength. In Chapter 4, we present our numerical analysis of a hybrid approach that combines a buried $\mathrm{Si}_{3} \mathrm{~N}_{4}$ 
waveguide with a thin piezoelectric layer to generate a Rayleigh-type surface acoustic wave. The SAW-induced modulation of the effective linear refractive index is calculated to show that an intensity modulator with high modulation frequency based upon a Mach-Zehnder interferometer should be realizable in the $\mathrm{Si}_{3} \mathrm{~N}_{4}$ platform considered here. In Chapter 5, we present our results on second-harmonic generation in $\mathrm{Si}_{3} \mathrm{~N}_{4}$ via the coherent photogalvanic effect which shows that the symmetry of the normally amorphous can be broken for implementing second-order nonlinear processes. Finally, in Chapter 6, we provide a summary of our work together with conclusions and an outlook.

\section{References}

1. European Technology Platform Photonics21. Photonics: A Key Enabling Technology of Europe (Photonics21, 2013).

2. NPI. Photonics: enabling American innovation, competition and security (2017).

3. Kao, K. C. \& Hockham, G. A. Dielectric-fibre surface waveguides for optical frequencies. Proc. of IEEE 113, 1151-1158 (1966).

4. Schawlow, A. L. \& Townes, C. H. Infrared and Optical Masers. Phys. Rev. 112, 19401949 (1958).

5. Maiman, T. H. Optical and Microwave-Optical Experiments in Ruby. Phys. Rev. Lett. 4, 564-566 (1960).

6. Cocito, G., Costa, B., Longoni, S., Michetti, L., Silvestri, L., Tibone, D. \& Tosco, F. COS 2 experiment in Turin: Field test on an optical cable in ducts. IEEE Trans. Commun. 26, 1028-1036 (1978).

7. Hecht, J. City of Light: The Story of Fiber Optics Revised and Expanded Edition (Oxford University Press, Oxford, New York, 2004).

8. Marcatili, E. A. Dielectric rectangular waveguide and directional coupler for integrated optics. Bell Syst. Tech. J. 48, 2071-2102 (1969).

9. Miller, S. E. Integrated optics: an introduction. Bell Syst. Tech. J. 48, 1538-7305 (1969).

10. Chang, L., Pfeiffer, M. H. P., Volet, N., Zervas, M., Peters, J. D., Manganelli, C. L., Stanton, E. J., Li, Y., Kippenberg, T. J. \& Bowers, J. E. Heterogeneous integration of lithium niobate and silicon nitride waveguides for wafer-scale photonic integrated circuits on silicon. Opt. Lett. 42, 803-806 (2017).

11. Stegeman, G. I. \& Seaton, C. T. Nonlinear integrated optics. J. Appl. Phys. 58, 57-78 (1985).

12. Moss, D. J., Morandotti, R., Gaeta, A. L. \& Lipson, M. New CMOS-compatible platforms based on silicon nitride and Hydex for nonlinear optics. Nat. Photon. 7, 597-607 (2013).

13. Estevez, M., Alvarez, M. \& Lechuga, L. Integrated optical devices for lab-on-a-chip biosensing applications. Laser Photon. Rev. 6, 463-487 (2012). 
14. Llobera, A., Juvert, J., González-Fernández, A., Ibarlucea, B., Carregal-Romero, E., Büttgenbach, S. \& Fernández-Sánchez, C. Biofunctionalized all-polymer photonic lab on a chip with integrated solid-state light emitter. Light Sci. Appl. 4, e271 (2015).

15. Mentzer, M. A. Applied Optics Fundamentals and Device Applications: Nano, MOEMS, and Biotechnology (CRC Press, 2016).

16. Syms, R. R. A. \& Cozens, J. R. Optical guided waves and devices (McGraw-Hill, London ; New York, 1992).

17. Dorf, R. C. Broadcasting and Optical Communication Technology (CRC Press, 2006).

18. Marpaung, D., Roeloffzen, C., Heideman, R., Leinse, A., Sales, S. \& Capmany, J. Integrated microwave photonics. Laser Photon. Rev. 7, 506-538 (2013).

19. Capmany, J. \& Novak, D. Microwave photonics combines two worlds. Nat. Photon. 1, 319-330 (2007).

20. Oldenbeuving, R. M., Klein, E. J., Offerhaus, H. L., Lee, C. J., Song, H. \& Boller, K.-J. $25 \mathrm{kHz}$ narrow spectral bandwidth of a wavelength tunable diode laser with a short waveguide-based external cavity. Laser Phys. Lett. 10, 015804 (2013).

21. Fan, Y., Epping, J. P., Oldenbeuving, R. M., Roeloffzen, C. G. H., Hoekman, M., Dekker, R., Heideman, R. G., van der Slot, P. J. M. \& Boller, K.-J. Optically Integrated InP-Si ${ }_{3} \mathrm{~N}_{4}$ Hybrid Laser. IEEE Photonc J. 8, 1-11 (2016).

22. Zhuang, L., Roeloffzen, C. G. H., Hoekman, M., Boller, K.-J. \& Lowery, A. J. Programmable photonic signal processor chip for radiofrequency applications. Optica 2, 854-859 (2015).

23. Epping, J. P. et al. On-chip visible-to-infrared supercontinuum generation with more than $495 \mathrm{THz}$ spectral bandwidth. Opt. Express 23, 19596-19604 (2015).

24. Ferdous, F., Miao, H., Leaird, D. E., Srinivasan, K., Wang, J., Chen, L., Varghese, L. T. \& Weiner, A. M. Spectral line-by-line pulse shaping of on-chip microresonator frequency combs. Nat. Photon. 5, 770-776 (2011).

25. Boyd, R. W. Nonlinear Optics (Academic Press, 2013).

26. Stegeman, G. I., Hagan, D. J. \& Torner, L. $\chi^{(2)}$ cascading phenomena and their applications to all-optical signal processing, mode-locking, pulse compression and solitons. Opt. Quantum Electron. 28, 1691-1740 (1996).

27. Korpel, A. Acousto-Optics, Second Edition (CRC Press, 1996).

28. Sutherland, R. L. Handbook of Nonlinear Optics (Taylor \& Francis, 1996).

29. Hendrickson, S. M., Foster, A. C., Camacho, R. M. \& Clader, B. D. Integrated nonlinear photonics: emerging applications and ongoing challenges. J. Opt. Soc. Am. B 31, 31933203 (2014).

30. Tan, D. T. H., Ikeda, K., Sun, P. C. \& Fainman, Y. Group velocity dispersion and self phase modulation in silicon nitride waveguides. Appl. Phys. Lett. 96, 061101 (2010).

31. Chavez Boggio, J. M., Bodenmüller, D., Fremberg, T., Haynes, R., Roth, M. M., Eisermann, R., Lisker, M., Zimmermann, L. \& Böhm, M. Dispersion engineered silicon nitride waveguides by geometrical and refractive-index optimization. J. Opt. Soc. Am. B 31, 2846 (2014).

32. Suhara, T. \& Fujimura, M. Waveguide Nonlinear-Optic Devices red. by Kamiya, T., Monemar, B., Venghaus, H. \& Yamamoto, Y. (Springer Berlin Heidelberg, Berlin, Heidelberg, 2003).

33. Bogaerts, W., Baets, R., Dumon, P., Wiaux, V., Beckx, S., Taillaert, D., Luyssaert, B., Campenhout, J. V., Bienstman, P. \& Thourhout, D. V. Nanophotonic waveguides in 
silicon-on-insulator fabricated with CMOS technology. J. Lightwave Technol. 23, 401412 (2005).

34. Granzow, N., Stark, S. P., Schmidt, M. A., Tverjanovich, A. S., Wondraczek, L. \& Russell, P. S. J. Supercontinuum generation in chalcogenide-silica step-index fibers. Opt. Express 19, 21003-21010 (2011).

35. Pavesi, L. Silicon Photonics (Springer Science \& Business Media, 2004).

36. Levy, J. S., Gondarenko, A., Foster, M. A., Turner-Foster, A. C., Gaeta, A. L. \& Lipson, M. CMOS-compatible multiple-wavelength oscillator for on-chip optical interconnects. Nat. Photon. 4, 37-40 (2010).

37. Agrawal, G. P. Nonlinear Fiber Optics (Academic Press, 2007).

38. Liu, X.-J., Zhang, J.-J., Sun, X.-W., Pan, Y.-B., Huang, L.-P. \& Jin, C.-Y. Growth and properties of silicon nitride films prepared by low pressure chemical vapor deposition using trichlorosilane and ammonia. Thin Solid Films 460, 72-77 (2004).

39. Bauters, J. F., Heck, M. J. R., John, D., Dai, D., Tien, M.-C., Barton, J. S., Leinse, A., Heideman, R. G., Blumenthal, D. J. \& Bowers, J. E. Ultra-low-loss high-aspect-ratio $\mathrm{Si}_{3} \mathrm{~N}_{4}$ waveguides. Opt. Express 19, 3163-3174 (2011).

40. Romero-García, S., Merget, F., Zhong, F., Finkelstein, H. \& Witzens, J. Silicon nitride CMOS-compatible platform for integrated photonics applications at visible wavelengths. Opt. Express 21, 14036 (2013).

41. Wörhoff, K., Heideman, R. G., Leinse, A. \& Hoekman, M. TriPleX: a versatile dielectric photonic platform. Adv. Opt. Technol. 4, 189-207 (2015).

42. Luke, K., Okawachi, Y., Lamont, M. R. E., Gaeta, A. L. \& Lipson, M. Broadband midinfrared frequency comb generation in a $\mathrm{Si}_{3} \mathrm{~N}_{4}$ microresonator. Opt. Lett. 40, 4823-4826 (2015).

43. Kitao, A., Imakita, K., Kawamura, I. \& Fujii, M. An investigation into second harmonic generation by Si-rich $\mathrm{SiN}$ thin films deposited by RF sputtering over a wide range of Si concentrations. J. Phys. D: Appl. Phys. 47, 215101 (2014).

44. Krückel, C. J., Torres-Company, V., Andrekson, P. A., Spencer, D. T., Bauters, J. F., Heck, M. J. R. \& Bowers, J. E. Continuous wave-pumped wavelength conversion in low-loss silicon nitride waveguides. Opt. Lett. 40, 875 (2015).

45. Krückel, C. J., Fülöp, A., Ye, Z., Andrekson, P. A. \& Torres-Company, V. Optical bandgap engineering in nonlinear silicon nitride waveguides. Opt. Express 25, 15370 (2017).

46. Epping, J. P., Hoekman, M., Mateman, R., Leinse, A., Heideman, R. G., van Rees, A., van der Slot, P. J. M., Lee, C. J. \& Boller, K.-J. High confinement, high yield $\mathrm{Si}_{3} \mathrm{~N}_{4}$ waveguides for nonlinear optical applications. Opt. Express 23, 642-648 (2015).

47. Pfeiffer, M. H. P., Kordts, A., Brasch, V., Lecaplain, C., Jost, J. D., Geiselmann, M. \& Kippenberg, T. J. Photonic damascence process for high-Q SiN microresonator fabrication for nonlinear photonics in 2015 Conference on Lasers and Electro-Optics (CLEO) (2015), 1-2.

48. Epping, J. P., Kues, M., van der Slot, P. J. M., Lee, C. J., Fallnich, C. \& Boller, K.-J. Integrated CARS source based on seeded four-wave mixing in silicon nitride. Opt. Express 21, 32123-32129 (2013).

49. Mayer, A. S., Klenner, A., Johnson, A. R., Luke, K., Lamont, M. R. E., Okawachi, Y., Lipson, M., Gaeta, A. L. \& Keller, U. Frequency comb offset detection using supercontinuum generation in silicon nitride waveguides. Opt. Express 23, 15440 (2015). 
50. Margulis, W. \& Österberg, U. Second-harmonic generation in optical glass fibers. J. Opt. Soc. Am. B 5, 312-316 (1988).

51. Martin, W. E. A new waveguide switch/modulator for integrated optics. Appl. Phys. Lett. 26, 562-564 (1975).

52. Ramaswamy, V., Divino, M. D. \& Standley, R. D. Balanced bridge modulator switch using Ti-diffused $\mathrm{LiNbO}_{3}$ strip waveguides. Appl. Phys. Lett. 32, 644-646 (1978). 

Chapter 2

Theory: nonlinear optics in amorphous waveguides 
Nowadays, integrated optics, which is also called integrated photonics, usually refers to optical circuits realized on a chip, similar to an electronic integrated circuit. In this Chapter, we present the theory for waveguiding and nonlinear optical processes in dielectric waveguides made of amorphous material, in particular $\mathrm{Si}_{3} \mathrm{~N}_{4} / \mathrm{SiO}_{2}$ waveguides, as relevant for this thesis.

\subsection{Waveguide optics}

One of the most basic elements of integrated photonics are optical waveguides that can confine and transport a light wave over extended distances. A typical example of an integrated dielectric optical waveguide is shown in Fig. 2.1, which comprises a rectangular core with refractive index $n_{c}$, surrounded by a cladding with refractive index $n_{s}$. Optical guiding along the longitudinal, $z$-direction is obtained when $n_{c}>n_{s}$, and low-loss guiding requires that both materials are sufficiently transparent for the light wave. Some of the characteristics of wave guiding in dielectric

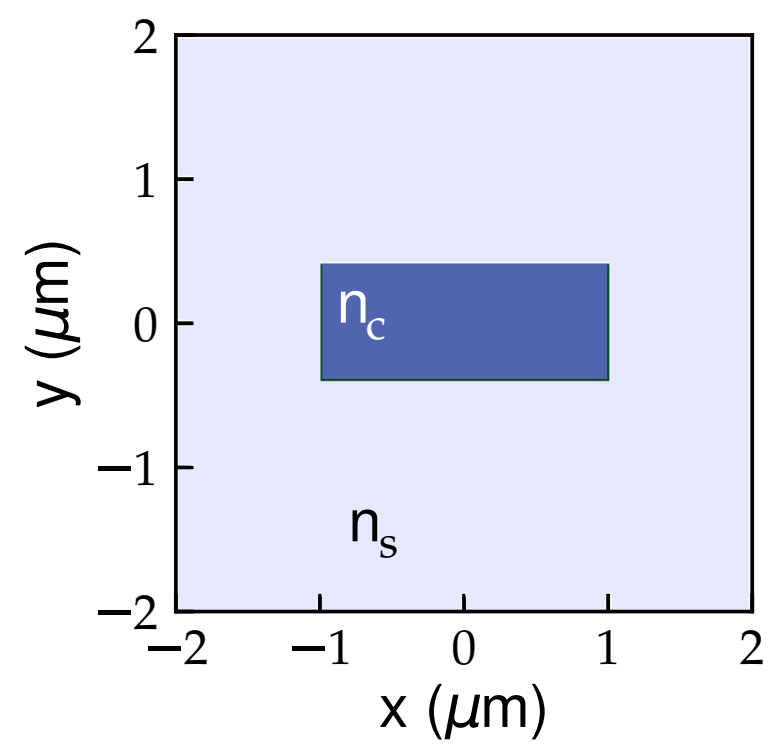

Figure 2.1: Cross section of a typical dielectric optical waveguide consisting of a core surrounded by a cladding. The refractive index, $n_{c}$, of the core needs to be higher than the refractive index, $n_{s}$, of the cladding to obtain optical guiding long the longitudinal direction $(z)$.

waveguides can be understood using a ray optics picture [1]. However, a complete description of light guiding is only obtained when taking into account the wave nature of light [2] as achieved using Maxwell's equations [2,3]. For recalling the basic principles of optical waveguiding, let us consider a monochromatic (time-harmonic) 
electromagnetic field $\tilde{\mathbf{E}}$ given by

$$
\tilde{\mathbf{E}}(\mathbf{r}, t)=\mathbf{E}(\mathbf{r}) e^{j \omega t}+\mathbf{E}^{*}(\mathbf{r}) e^{-j \omega t},
$$

where $\mathbf{E}(\mathbf{r})$ is the complex amplitude of the electric field that depends on the positional vector $\mathbf{r}, \omega$ is the angular frequency, $t$ is time. The electric field is accompanied with a corresponding magnetic field $\tilde{\mathbf{H}}(\mathbf{r}, t)$. In absence of free charges and currents, which is usually appropriate for dielectric waveguides, Maxwell's equations for the time-harmonic fields reduce to a space-dependent set of equations,

$$
\begin{aligned}
\nabla \times \mathbf{E}(\mathbf{r}) & =-j \omega \mu_{0} \mathbf{H}(\mathbf{r}) \\
\nabla \times \mathbf{H}(\mathbf{r}) & =j \omega \varepsilon_{0} \varepsilon \mathbf{E}(\mathbf{r}) \\
\nabla \cdot \mathbf{E}(\mathbf{r}) & =0 \\
\nabla \cdot \mathbf{H}(\mathbf{r}) & =0 .
\end{aligned}
$$

Here, $\varepsilon$ is the relative dielectric permitivity, $\varepsilon_{0}$ and $\mu_{0}$ are the permitivity and the permeability of vacuum, respectively. The relative permeability is set to unity because here we consider dielectric waveguides that are fabricated from non-magnetic materials. In an isotropic homogeneous medium, Maxwell's equations can be combined to form the Helmholtz equation,

$$
\nabla^{2} \mathbf{E}(\mathbf{r})+k^{2} \varepsilon \mathbf{E}(\mathbf{r})=0,
$$

where $k=\omega \sqrt{\varepsilon_{0} \mu_{0}}=\omega / c=2 \pi / \lambda$ is the wavenumber, $c$ is the speed of light in vacuum and $\lambda$ is the wavelength in vacuum. An equation similar to Eq. 2.3 governs also the space dependence of the complex amplitude of the magnetic field, $\mathbf{H}(\mathbf{r})$. Throughout this work, $\lambda$ and $c$ will remain denoting the wavelength and speed of the light in vacuum, respectively.

We take the $z$-axis to be directed along the axis of the dielectric waveguide as shown in Fig. 2.1, and assume that the index cross-section does not change with $z$. Consequently, the index of refraction is a function only of the transverse coordinates $x$ and $y, n(x, y)=\sqrt{\varepsilon(x, y)}$. Given such an index profile, the solution to Eq. 2.3 in a lossless medium (real-valued $k$ and $\varepsilon_{0}$ ) can be written as the product of a transverse and longitudinal part,

$$
\mathbf{E}(\mathbf{r})=\mathbf{E}(x, y) e^{-j \beta z},
$$

where $\beta$ is the longitudinal propagation constant of the wave in the waveguide. A similar equation is obtained for the complex amplitude of the magnetic field. Substituting Eq. 2.4 into the Helmholtz equation (Eq. 2.3) gives the conditions that waveguiding imposes on $\beta$,

$$
\left(\nabla_{t}^{2}+\left(k^{2} n^{2}(x, y)-\beta^{2}\right)\right) \mathbf{E}(x, y)=0
$$


Here $\nabla_{t}^{2}$ is the transverse Laplace operator and an equivalent equation governs $\mathbf{H}(x, y)$. In general $\beta$ is bigger than $k$ (the vacuum propagation wavenumber). At this point it is standard to define the effective refractive index as the ratio of the two, $n_{\text {eff }}=\beta / k$.

Finding the transverse field distribution, $\mathbf{E}(x, y)$, in Eq 2.5 and the corresponding value for $\beta$ with a given cross section is known as an eigenvalue problem. The solutions for each of the eigenvalues characterized by $\beta$ are known as eigenmodes (or transverse modes), in this case the transverse distribution of the complex amplitude $\mathbf{E}(x, y)$. As the set of all eigenmodes is a complete set [2], any transverse distribution of an electric field can be written as a superposition of eigenmodes

$$
\mathbf{E}(x, y, z)=\sum_{m} \sum_{n} \mathcal{A}_{m n} \mathbf{E}_{m n}(x, y) e^{-j \beta_{m n} z},
$$

where $m$ and $n$ label the order of the mode along the $x$ - and $y$-directions, respectively, $\mathcal{A}_{m n}$ is the amplitude of the mode and $\mathbf{E}_{m n}$ determines the polarization and the transverse field distribution. The lowest-order propagating mode $(m=n=1)$ is also known as the fundamental or fundamental transverse mode. Modes with $m$ or $n>1$ are called higher-order or transverse modes.

In general, Eq. 2.5 cannot be solved analytically, even for a simple refractive index distribution as shown in Fig. 2.1. Approximate solutions may be obtained making use of the Marcatili's method [4] that considers separate solutions for the two transverse directions. However, for proper design and optimization of linear and nonlinear processes in waveguides, a more accurate solution is required. Several numerical methods can be employed, mainly based on the finite element method (FEM) [5] or on the finite difference method (FDM) [6].

Figure 2.2 shows a typical example of a numerically calculated intensity distribution normalized to its maximum value for the fundamental mode and a polarization in the $x$-direction (a) and $y$-direction (b). In this example, the waveguide is considered to comprise of a stoichiometric $\mathrm{Si}_{3} \mathrm{~N}_{4}$ core with a width of $2.0 \mu \mathrm{m}$ and a height of $0.8 \mu \mathrm{m}$, surrounded by a $\mathrm{SiO}_{2}$ cladding. These materials and dimensions are taken as example because the main chapters of the thesis are based on such waveguides and similar dimensions. To avoid non-physical reflections, the simulation domain is taken sufficiently large to have negligible optical field at the domain boundary. The refractive index for the core, $n_{c}=n_{S i_{3} N_{4}}$, is given by the Sellmeier equation [7]

$$
n_{\mathrm{Si}_{3} \mathrm{~N}_{4}}^{2}(\lambda)=1+\frac{3.0249 \lambda^{2}}{\lambda^{2}-0.1353406^{2}}+\frac{40314 \lambda^{2}}{\lambda^{2}-1239.842^{2}},
$$

while the refractive index for the cladding, $n_{s}=n_{\mathrm{SiO}_{2}}$, is given by [8]

$$
n_{\mathrm{SiO}_{2}}^{2}(\lambda)=1+\frac{0.6961663 \lambda^{2}}{\lambda^{2}-0.0684043^{2}}+\frac{0.4079426 \lambda^{2}}{\lambda^{2}-0.1162414^{2}}+\frac{0.8974794 \lambda^{2}}{\lambda^{2}-9.896161^{2}} .
$$


In Eqs. 2.7 and 2.8 the vacuum wavelength, $\lambda$, must be specified in units of $\mu \mathrm{m}$. The value of the effective refractive index for the shown modes, calculated as $n_{\text {eff }}=\beta / k$, is displayed in each figure.
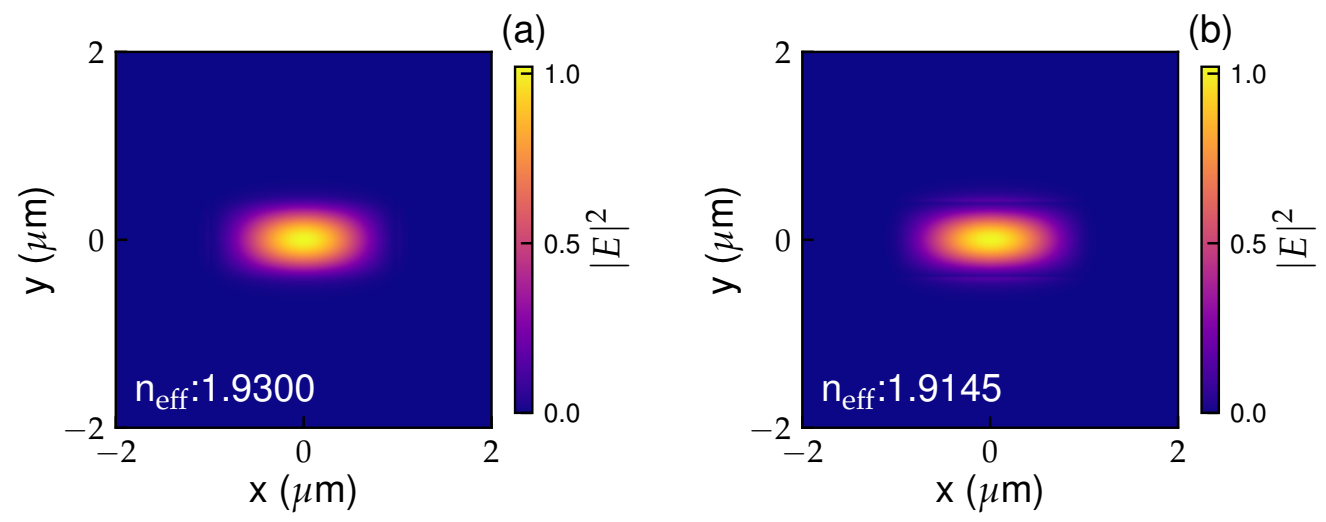

Figure 2.2: Normalized intensity distribution (profile), which is proportional to $|\mathbf{E}|^{2}$, for a waveguide core with a width of $2 \mu \mathrm{m}$ and a height of $0.8 \mu \mathrm{m}$, (a) for the fundamental mode polarized in the $x$ direction, $\mathrm{E}_{11, x}$, or quasi-TE mode, and (b) in the $y$-direction, $\mathrm{E}_{11, y}$, or quasi-TM mode, both at the wavelength of $1064 \mathrm{~nm}$.

By inspecting the three Cartesian components of the electric field of the fundamental mode, it can be seen that the electric field distribution points either dominantly in the $x$-direction or the $y$-direction, with only a small component in the $z$-direction. Accordingly, the polarization of the fundamental mode is labeled as $\mathrm{E}_{11, x}$, quasi-TE or TE-like, or as $\mathrm{E}_{11, y}$, quasi-TM or TM-like, respectively [2]. For higher-order modes the longitudinal electric field, $E_{z}$, can be of comparable amplitude. These modes are typically labeled as $\mathrm{E}_{m n, p}$ where $m$ and $n$ are the two modes indices that corresponds to the number of maxima in the electric field in the $x$ - and $y$-direction, respectively, and $p$ indicates again the transverse polarization direction.

Examples of the normalized intensity profiles for the next two higher-order modes for the same waveguide configuration and wavelength as was used for Fig. 2.2 are shown in Fig. 2.3. This figure shows the normalized intensity profile for the secondorder mode with $x$-polarization, $\mathrm{E}_{21, x}$, (a) and $y$-polarization, $\mathrm{E}_{21, y}$, (b) and for the third-order mode with $x$-polarization, $\mathrm{E}_{31, x},(\mathrm{c})$ and $y$-polarization, $\mathrm{E}_{31, y}(\mathrm{~d})$. For each of the modes, the effective refractive index is calculated and the values are shown in each of the figures. As expected the effective refractive index is different for each of the modes, which is known as modal dispersion. For increasing order of the mode the effective refractive index is decreasing, while the $y$-polarization has a slightly higher effective refractive index than the $x$-polarization for each of the modes. The number of transverse modes that exists for a given wavelength is determined by the geometry of the waveguides and the refractive indices of the core 

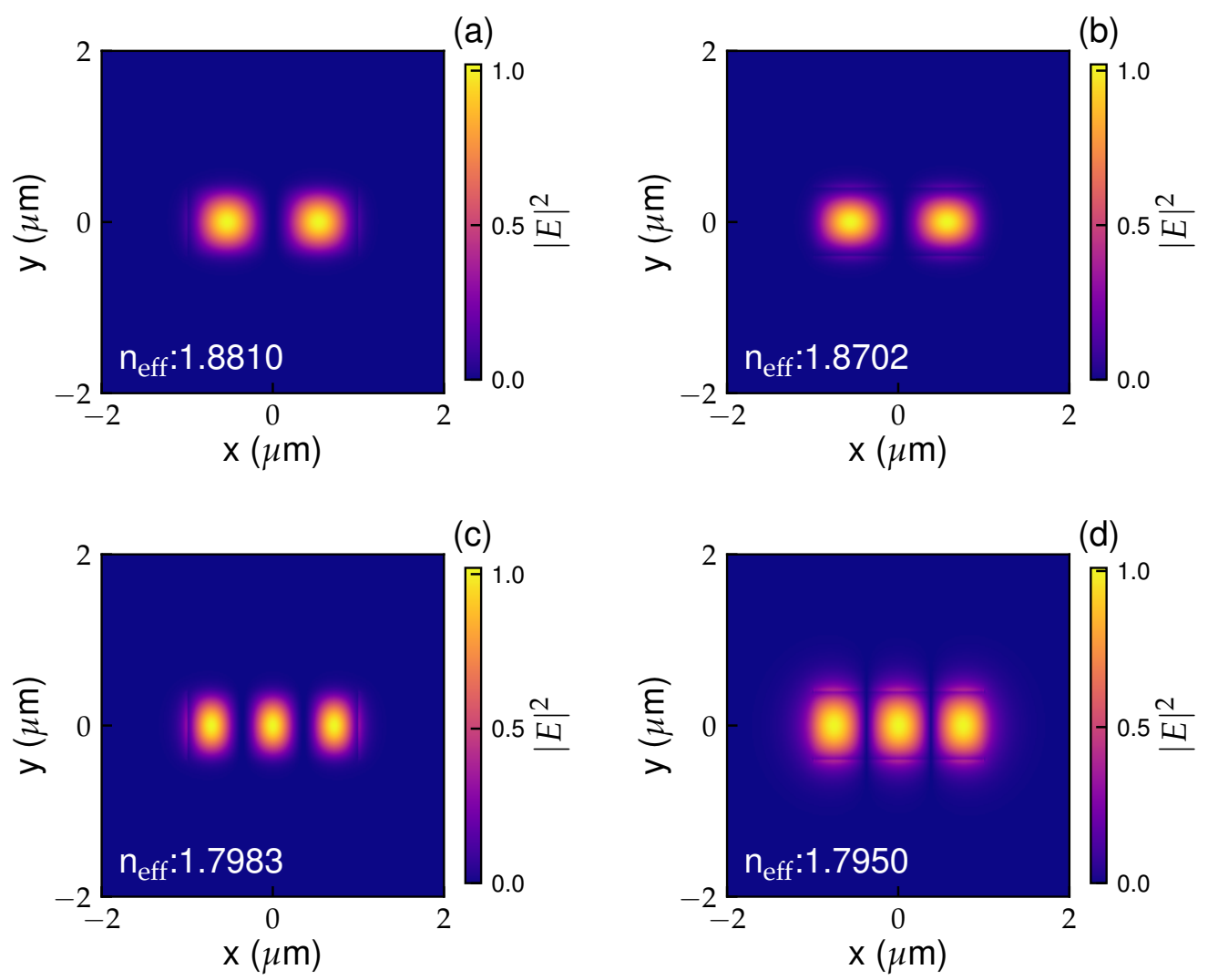

Figure 2.3: Normalized intensity profile at a wavelength of $1064 \mathrm{~nm}$ for a waveguide core width of $2 \mu \mathrm{m}$ and a height of $0.8 \mu \mathrm{m}$ for the second-order $\mathrm{E}_{21, x}$ (a) and third-order $\mathrm{E}_{31, x}$ (c) modes with horizontal polarization and the same modes with vertical polarization, (b) and (d), respectively.

and cladding [2]. When the waveguide only supports the fundamental mode, it is called a single-mode waveguide.

\subsection{Dispersion}

Dispersion describes how the phase velocity of a wave, as it travels through media, depends on frequency (or wavelength) of the wave. This is known as chromatic dispersion. When light propagates through a homogeneous material, the dispersion is set solely by the material properties, specifically the linear susceptibility or, alternatively, the dielectric constant or refractive index [9] which determines the phase velocity. In case of propagation of short pulses, due to their broader spectral content, 
also the group velocity is of interest and the change of group velocity with frequency is called group velocity dispersion [1,9]. As dispersion plays an important role in phase matching nonlinear optical conversion [9], which is the subject of two chapters in this thesis, we will briefly discuss how the dispersion can be controlled or engineered in case of waveguiding using dielectric waveguides.

In this work we consider guided wave propagation in stoichiometric silicon nitride waveguides and, consequently, the material dispersion is set by the corresponding Sellmeier equations 2.7 and 2.8 for the core and cladding, respectively. However, the propagation constants of the modes that are solutions of Eq. 2.5 depend also on the geometry of the waveguide and the wavelength and polarization of the mode. By varying the dimensions of the waveguide core and choosing a particular polarization the total dispersion, which is a combination of the material, modal and polarization mode dispersion, can be engineered to assume certain required properties.

As an example, Fig. 2.4 shows $n_{\text {eff }}$ as a function of the width of the core for $x$ polarized light for the fundamental and various higher-order transverse modes at a fixed wavelength of $\lambda=1064 \mathrm{~nm}$ and a constant core height of $0.8 \mu \mathrm{m}$. Figure 2.4 shows the modal dispersion, and one can see that the fundamental $\mathrm{E}_{11,(x, y)}$ mode has the highest effective refractive index for all widths investigated. We also observe that, for each of the modes, $n_{\text {eff }}$ decreases monotonically with decreasing width of the core until it becomes equal to the cladding's refractive index and the mode is not guided any more. This means that for sufficiently small widths of the core, the waveguide becomes single mode, only guiding the fundamental mode. On the contrary, the number of transverse modes supported by the waveguide increases with the width of the core. A physical interpretation is that with increasing area of the core, the transverse profile of the mode is increasingly more confined to the core and the optical field experiences to an increasing extent only of the refractive index of the core, letting $n_{\text {eff }}$ approaching the refractive index of the core. To quantify the degree of confinement or size of a mode it is standard to define the effective area, $A_{\text {eff }}$, of the transverse profile of the optical mode as

$$
A_{\mathrm{eff}}=\frac{\left(\iint|E|^{2} d x d y\right)^{2}}{\left.\iint|E|^{4} d x d y\right)} .
$$

For increasing size more of the optical field penetrates into the cladding and $n_{\text {eff }}$ approaches the refractive index of the cladding, because $A_{\text {eff }}$ becomes much larger than the core area.

To illustrate the effect of chromatic dispersion of the effective refractive index, we display in Fig. $2.5 n_{\text {eff }}$ as a function of wavelength for a rectangular dielectric waveguide as shown in Fig. 2.1 with a fixed height of $0.8 \mu \mathrm{m}$ and for three different core widths of 0.2 (solid blue line), 0.4 (solid orange line) and $0.6 \mu \mathrm{m}$ (solid green line). 


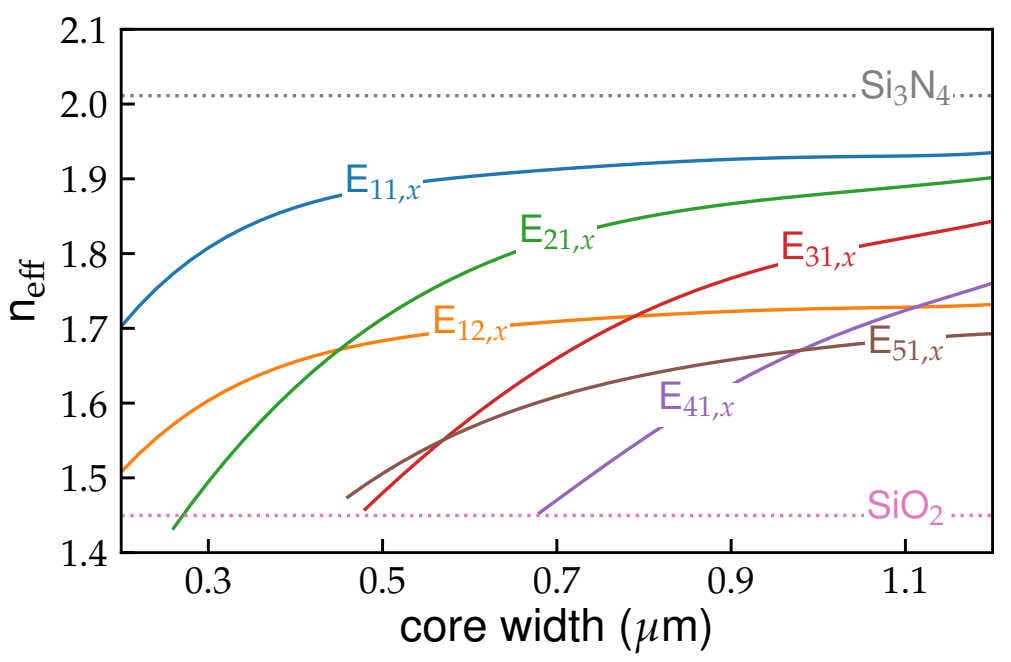

Figure 2.4: $n_{\mathrm{eff}}$ as a function of the width of the waveguide core for $x$-polarized light at the fundamental and various higher-order modes. The waveguide core has a fixed height of $0.8 \mu \mathrm{m}$ and the wavelength is $1064 \mathrm{~nm}$. The dotted traces represent the refractive index of cladding (pink) and core (gray) at the given wavelength.

The light is assumed to be guided in the fundamental mode and polarized in the $x$-direction. The purple and red dotted lines represent the bulk refractive indices of the core and cladding materials. Figure 2.5 shows that for small wavelengths $n_{\text {eff }}$ approaches the core refractive index, while for the large wavelengths $n_{\text {eff }}$ approaches the cladding index. The physical interpretation is again that, as the wavelength gets smaller, the light becomes more confined to the core of the waveguide while as, the wavelength gets longer, the light penetrates more into the cladding. Figure 2.5 also shows that, with increasing core area, the largest and smallest wavelengths where the light can be considered to be confined to the core and almost completely resides in the cladding, respectively, shift to longer wavelengths.

Figures 2.4 and 2.5 illustrate that $n_{\text {eff }}$ can be engineered by choosing appropriate dimensions of the waveguide, in combination with choosing a particular transverse mode of the light. In these figures we have plotted $n_{\mathrm{eff}}$ as a function of the vacuum wavelength, however, from a modeling point of view, it is more convenient to describe the dispersion as a function of the angular frequency, $\omega=c k=2 \pi c / \lambda$. In order to represent the dispersion in a mathematical model for describing nonlinear optical pulse propagation, it is convenient to approximate the frequency variation of the propagation constant by a polynomial fit via a Taylor expansion around the 


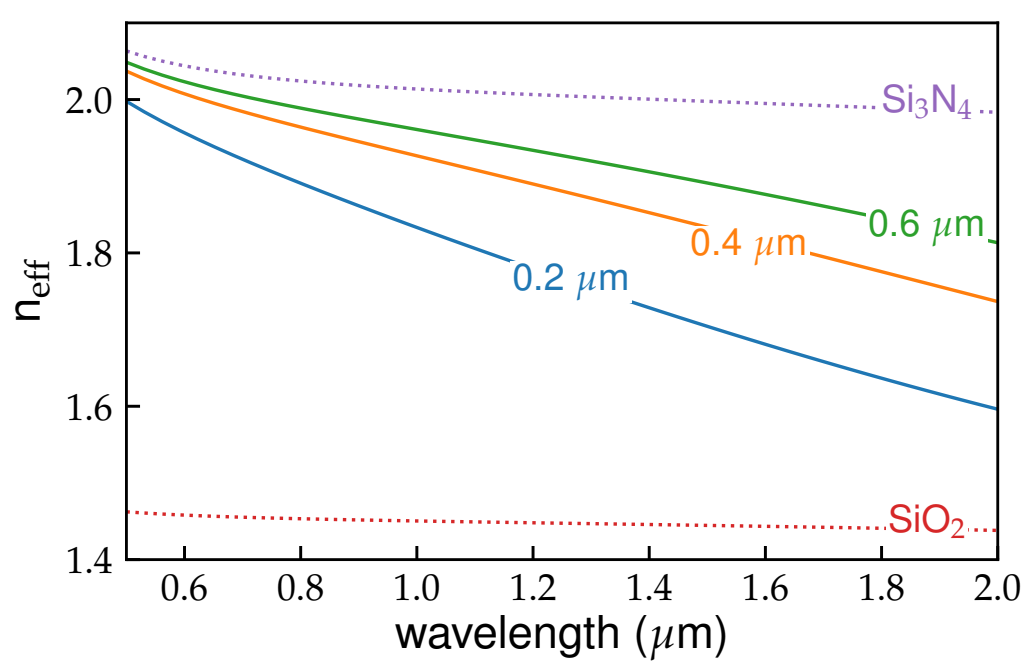

Figure 2.5: $n_{\text {eff }}$ as a function of the vacuum wavelength for the fundamental mode polarized in the $x$-direction for a rectangular waveguide with a constant height of $0.8 \mu \mathrm{m}$ and three widths equal to 0.2 (solid blue line), 0.4 (solid orange line) and $0.6 \mu \mathrm{m}$ (solid green line). The purple and red dotted lines represent the refractive index of bulk $\mathrm{Si}_{3} \mathrm{~N}_{4}$ and $\mathrm{SiO}_{2}$, respectively.

carrier frequency, $\omega_{0}$, of the optical pulse [9],

$$
\beta(\omega)=\beta_{0}+\beta_{1}\left(\omega-\omega_{0}\right)+\frac{1}{2} \beta_{2}\left(\omega-\omega_{0}\right)^{2}+\frac{1}{6} \beta_{3}\left(\omega-\omega_{0}\right)^{3}+\ldots
$$

Here $\beta_{m}$ is the $\mathrm{m}$-th order slope or curvature of the propagation constant at the carrier frequency given by [9]

$$
\beta_{m}=\left(d^{m} \beta / d \omega^{m}\right)_{\omega_{0}} .
$$

Specifically, the model describing supercontinuum generation (Chapter 3) uses this approximation for the propagation constant as a function of the angular frequency, and it turned out that an accurate modeling of the supercontinuum generation process may require an expansion beyond even the 19-th order. The second term in Eq. 2.10, $\beta_{1}$, has a direct physical meaning - it is the reciprocal of the group velocity at the carrier frequency, $v_{g}\left(\omega_{0}\right), \beta_{1}=(d \beta / d \omega)_{\omega_{0}}=1 / v_{g}\left(\omega_{0}\right)$. The next-higher order in Eq. 2.10 is known as the group velocity dispersion, GVD or $D$, defined as [9]

$$
D(\omega)=\frac{\partial}{\partial \omega} \frac{1}{v_{g}} .
$$


As the effective refractive index and propagation constant are often expressed as a function of the vacuum wavelength, $\lambda$, it is convenient to express Eq. 2.12 in terms of physical quantities that are function of $\lambda$,

$$
D(\lambda)=-\frac{2 \pi c}{\lambda^{2}} D(\omega)=-\frac{\lambda}{c} \frac{d^{2} n_{\mathrm{eff}}(\lambda)}{d \lambda^{2}} .
$$

When $D(\lambda)>0$, the dispersion is called anomalous, while $D(\lambda)<0$ is called normal dispersion. Anomalous dispersion plays an important role in soliton formation and supercontinuum generation [9, 10], which we briefly describe below. Higherorder terms in the Taylor expansion (Eq. 2.10) are commonly known as higher-order dispersion terms.

\subsection{Nonlinear optical polarization}

Nonlinear optical processes are driven by the nonlinear polarization generated by optical fields propagating through the medium. Having a small effective mode area for guided waves in PICs has the advantage that nonlinear optical processes can be efficiently driven by either a train of short pulses with a low average power or using resonant structures to enhance the local intensity of the light in the waveguide. In this section we briefly describe the nonlinear optical processes that are at the foundation of the experiments reported in Chapters 3 and 5.

The material response to an applied light field can be described in both the time and frequency domains [9]. Here, we choose to use the frequency domain description, while dependence on the positional vector $\mathbf{r}$ is implicitly assumed. Every dielectric medium develops an induced polarization wave, $\mathbf{P}(\omega)$, as a response to the electric field component of an injected light wave. For a monochromatic wave of angular frequency $\omega$, characterized by its electric field, $\mathbf{E}(\omega)$, the polarization wave can be written as [9]

$$
\mathbf{P}(\omega)=\mathbf{P}_{L}(\omega)+\mathbf{P}_{N L}(\omega)=\sum_{q} \mathbf{P}^{(q)}(\omega)
$$

where $\mathbf{P}_{L}(\omega)$ and $\mathbf{P}_{N L}(\omega)$ are the linear and nonlinear polarization contributions of the medium, respectively, and $\mathbf{P}^{(q)}(\omega)$ is the polarization of order $q$ defined as [9]

$$
\mathbf{P}^{(q)}(\omega)=\varepsilon_{0} \sum_{q} \chi^{(q)}\left(\omega_{1}, \ldots, \omega_{q} ; \omega\right) \mathbf{E}_{1}\left(\omega_{1}\right) \cdots \mathbf{E}_{q}\left(\omega_{q}\right)
$$

Here, $\chi^{(q)}\left(\omega_{1}, \ldots, \omega_{q} ; \omega\right)$ is the susceptibility tensor for the medium of order $q$ for the optical process that generates frequency $\omega$ from the frequencies $\omega_{1}, \ldots, \omega_{q}$. The vector Eqs. 2.14 and 2.15 can be reduced to a scalar expression when the material is 
known and the propagation direction of the fields and the orientation of the polarization of all the frequency components are known.

For most of the nonlinear optical processes, the magnitude of the higher-order susceptibility reduces strongly with increasing order. For example, in a nonlinear, noncentrosymmetric material, usually crystalline, the strongest nonlinear response is that of the second order $(q=2)$. In that case

$$
\mathbf{P}^{(2)}(\omega)=\varepsilon_{0} \chi^{(2)}\left(\omega_{1}, \omega_{2} ; \omega\right) \mathbf{E}\left(\omega_{1}\right) \mathbf{E}\left(\omega_{2}\right) .
$$

In general, eq. 2.16 is applicable to various different second-order nonlinear processes where the two frequencies $\omega_{1}$ and $\omega_{2}$ generate a new frequency $\omega$, however, each of these processes have their own second-order susceptibility tensor. The most common of these processes are sum-frequency generation (SFG), $\omega=\omega_{1}+\omega_{2}$, difference frequency generation (DFG), $\omega=\omega_{1}-\omega_{2}$, second-harmonic generation (SHG), $\omega=2 \omega_{2}$, and the electro-optic (or Pockels) effect, where one of the applied fields is static $\left(e . g, \omega_{1}=0\right)$, so that $\omega=\omega_{2}$. For SHG the two participating input fields are frequency degenerate (i.e, $\omega_{1}=\omega_{2}$ ) so that the relevant second-order susceptibility is $\chi^{(2)}\left(\omega_{1}, \omega_{1} ; \omega=2 \omega_{1}\right)$.

If three optical waves at the three frequencies $\omega, \omega_{1}$ and $\omega_{2}$ are incident to a medium with a non-zero second-order susceptibility, the question arises which of the possible second-order nonlinear processes will take place in the medium, i.e., which of the various nonlinear polarization waves will radiate (generate) a light wave. It turns out out that only those processes that are phase matched (see section 2.4) are able to efficiently generate the nonlinear frequency component.

To recall a most simple example, which is also of relevance in Chapter 5, we describe some central relations for second-harmonic generation where both optical fields at the fundamental frequency, $\omega$, and the second harmonic frequency, $2 \omega$, can each be written as one of the modes of Eq. 2.6,

$$
\begin{array}{r}
\mathbf{E}_{m n}(x, y, z ; \omega)=\mathcal{A}_{m n}(z ; \omega) \mathbf{E}_{m n}(x, y) \exp \left(-j \beta_{m n} z\right), \\
\mathbf{E}_{m^{\prime} n^{\prime}}(x, y, z ; 2 \omega)=\mathcal{A}_{m^{\prime} n^{\prime}}(z ; 2 \omega) \mathbf{E}_{m^{\prime} n^{\prime}}(x, y) \exp \left(-j \beta_{m^{\prime} n^{\prime}} z\right) .
\end{array}
$$

Using these fields to calculated the nonlinear polarization (Eq. 2.15) and using Eqs. 2.14 and 2.15 in the inhomogeneous Maxwell equations the so-called coupled-wave equations can be derived [11]:

$$
\begin{array}{r}
\frac{d}{d z} \mathcal{A}_{m n}(z ; \omega)=-j \kappa^{*} \mathcal{A}_{m n}(z ; \omega)^{*} \mathcal{A}_{m^{\prime} n^{\prime}}(z ; 2 \omega) \exp (-j \Delta \beta z), \\
\frac{d}{d z} \mathcal{A}_{m^{\prime} n^{\prime}}(z ; 2 \omega)=-j \kappa\left|\mathcal{A}_{m n}(z, \omega)\right|^{2} \exp (j \Delta \beta z),
\end{array}
$$

where $\kappa$ is a coefficient that describes the strength of nonlinear coupling between the two modes, and $\Delta \beta$ is the mismatch defined as

$$
\Delta \beta=\beta_{m^{\prime} n^{\prime}}(2 \omega)-2 \beta_{m n}(\omega) .
$$


Since we are dealing with guided waves, the propagation directions of the waves are fixed. Also the waveguide materials are fixed (given) such that the relevant secondorder susceptibility tensor reduces to a scalar parameter, $d_{e f f}$, which represents the effective nonlinearity for the given polarization of the two fields at the fundamental and second-harmonic frequencies. The nonlinear coupling in the case of SHG (Eq. 2.18) is defined as [11]:

$$
\kappa=\varepsilon_{0} \sqrt{\frac{(2 \omega)^{2}}{2\left(n_{e f f, m n}(\omega)\right)^{2} n_{e f f, m^{\prime} n^{\prime}}(2 \omega)}\left(\frac{\mu_{0}}{\varepsilon_{0}}\right)^{3 / 2} \frac{d_{e f f}^{2}}{S_{e f f}}},
$$

where $S_{\text {eff }}$ is the effective mode overlap defined as:

$$
S_{\mathrm{eff}}=\frac{\iint\left|E_{m^{\prime} n^{\prime}}(x, y ; 2 \omega)\right|^{2} \partial x \partial y\left[\iint\left|E_{m n}(x, y ; \omega)\right|^{2} \partial x \partial y\right]^{2}}{\left[\iint\left[E_{m^{\prime} n^{\prime}}(x, y, 2 \omega)\right]^{*}\left[E_{m n}(x, y, \omega)\right]^{2} \partial x \partial y\right]^{2}} .
$$

Solving the coupled-mode equations (Eq. 2.18) under the assumption of negligible pump depletion and assuming that at the waveguide only the fundamental harmonic $\left(A_{0}\right)$ is injected, the second-harmonic amplitude is found to be

$$
\mathcal{A}_{m^{\prime} n^{\prime}}(z ; 2 \omega)=-j \kappa A_{0}^{2} z \exp j \frac{1}{2} \Delta \beta z\left(\frac{\sin \left(\frac{1}{2} \Delta \beta z\right)}{\frac{1}{2} \Delta \beta z}\right) .
$$

Equation 2.22 shows that, in the case of perfect phase matching, $\Delta \beta=0$, the field grows linearly with $z$ and quadratically with $A_{0}$, and thus the second-harmonic power grows quadratically with both the propagation coordinate (length of the waveguide used) and the power injected at the fundamental frequency. This dependency allows determination of the nonlinear coupling coefficient, $\kappa$, by measuring the output power at the second harmonic frequency as a function of the input power at the fundamental frequency for a given length of the waveguide, $z=L$. If the modes at the fundamental and second-harmonic frequencies are known, Eqs. 2.20 and 2.21 can be used the determine the second-order nonlinear susceptibility, $\chi^{(2)}$, nonlinearity of the waveguide for this process.

\subsection{Phase matching}

The nonlinear SHG process discussed in Section 2.3 only shows an appreciable conversion efficiency when the phase mismatch is close to zero, $\Delta \beta=0$ (c.f. Eq. 2.22), however, this condition is generally not fulfilled. The reason is that the nonlinear polarization wave has a phase velocity given by the driving light wave at $\omega$, $v_{\phi}=2 \omega / 2 \beta(\omega)$, while the optical wave to be radiated by the polarization wave (at 
the frequency $2 \omega$ ) has a different phase velocity $2 \omega / \beta(2 \omega)$. Due to this difference, which is caused by the chromatic material dispersion, the light field generated at the various positions along the waveguide will arrive with different phases at the end of the waveguide, which leads to destructive interference. Only when the two phase velocities are equal, all contribution generated along the length of the waveguide will constructively interfere at the exit of the waveguide, leading to a maximum conversion efficiency in SHG. Therefore, obtaining phase matching is a central goal and precondition in designing an efficient nonlinear optical process. The wave number mismatch, $\Delta \beta$, defines a coherence length, $\ell_{c}=\pi / \Delta \beta$, over which all contributions of the nonlinear polarization lead to an increase of the nonlinear field amplitude. From Fig. 2.5, which shows for the fundamental mode $E_{11, x}$ the material dispersion of the core and cladding and also the waveguide dispersion for different core dimensions, it is clear that the dispersion results in a large phase mismatch for SHG, because always $n_{\text {eff }}(\omega) \neq n_{\text {eff }}(2 \omega)$, or $n_{\text {eff }}(\lambda) \neq n_{\text {eff }}(\lambda / 2)$. Similarly, SHG from a higher-order transverse mode into the same transverse mode does not provide phase matching. On the other hand, Fig 2.4 shows that the different transverse modes can have significantly different phase velocities. Thereby, for certain dimensions of the waveguide, the two phase velocities can be made equal by selecting two different, specific, transverse modes for the two frequencies involved. The selections of the according core dimensions and transverse mode for SHG with $1064 \mathrm{~nm}$-radiation in $\mathrm{Si}_{3} \mathrm{~N}_{4}$ waveguides is presented in Chapter 5.

An alternative method for obtaining phase matching (QPM) is so-called quasi-phase matching (QPM) [9, 12]. For explaining the essence of quasi-phase matching it is instructive to divide the interaction length in sections with a length equal to the coherence length, $\ell_{c}$. Then one observes that contributions to the nonlinearly generated light field come from the sections which are located at an odd integer times the coherence length. Likewise the sections located at an even integer coherence lengths emit with the opposite phase, which leads to overall destructive interference. The idea of QPM is to suppress or phase-invert the generation of the nonlinear optical field for the section at the even multiple sections. Thereby destructive interference is avoided and the nonlinear optical field only grows steadily with $z$, although at a lower rate than for perfect phase matching. The highest output is achieved by flipping the sign of the nonlinear susceptibility, $\chi^{(2)}(z)$, every coherence length. This induces a phase jump of 180 degrees in the emission of the nonlinear field, bringing the contribution of the sections located at even multiples of the coherence length into phase with that of sections located at odd multiples of the coherence length. Mathematically in the coupled-wave equations 2.18, the described modulation of $\chi^{(2)} v s$. $z$ corresponds to providing what can be termed a $\chi^{(2)}$ grating of periodicity $\Lambda$. The periodicity of that grating extends the standard phase matching condition $(\Delta \beta$ in Eq. 2.19) with an extra wave vector, $K=2 \pi / \Lambda$. The phase matching condition for 
second-harmonic generation in the presence of a spatially patterned nonlinear susceptibility is then [12]

$$
\Delta \beta=\beta_{m^{\prime} n^{\prime}}(2 \omega)-2 \beta_{m n}(\omega)+K .
$$

The importance of this relation is, when standard phase matching cannot be obtained due to chromatic dispersion, still a suitable period of patterning $\chi^{(2)}$ may be realized, thereby providing quasi-phase matching (QPM, $\Delta \beta$ in Eq. 2.23). In waveguided SHG, choosing $\Lambda=2 \ell_{c}$, perfect quasi-phase matching can be obtained. It may then become possible to have the same transverse modes for the fundamental and second-harmonic frequencies, which would maximized the nonlinear coupling, according to Eq. 2.20.

\subsection{Nonlinear optics in amorphous materials}

Amorphous materials, specially most glasses, are centrosymmetric and therefore do not possess bulk even-order susceptibilities, such as a second-order susceptibility. In contrast, all materials possess odd-order susceptibilities, for which the strongest nonlinear response is obtained via the third-order susceptibility, $\chi^{(3)}$. The thirdorder nonlinear polarization is defined as $[9,11]$

$$
\mathbf{P}^{(3)}(\omega)=\varepsilon_{0} \chi^{(3)}\left(\omega_{1}, \omega_{2}, \omega_{3} ; \omega\right) \mathbf{E}\left(\omega_{1}\right) \mathbf{E}\left(\omega_{2}\right) \mathbf{E}\left(\omega_{3}\right) .
$$

where the nonlinear polarization at frequency $\omega$ is generated by three optical fields having frequencies $\omega_{1}, \omega_{2}$ and $\omega_{3}$. Again, the third-order nonlinear susceptibility supports various different types of third-order nonlinear optical processes, of which the Kerr effect, self-phase modulation (SPM), soliton formation and four-wave mixing (FWM) are the most relevant for this work.

Self-phase modulation is based on the Kerr effect, where the three optical input fields (and thus also the output field) all have the same frequency, $\omega_{1}=\omega_{2}=\omega_{3}=$ $\omega$. However, because the nonlinear polarization at $\omega$ is still experiencing a phase shift that grows with the input fields, this nonlinear process leads to an intensity dependent refractive index given by

$$
n(\omega)=n_{0}(\omega)+n_{2} I .
$$

Here $I \propto|\mathbf{E}|^{2}$ is the intensity of the optical field, $n_{0}$ is the linear material refractive index and $n_{2}=3 \chi^{(3)} /\left(4 n_{0}^{2} \varepsilon_{0} c\right)$ is the so-called Kerr index (Kerr coefficient) for a homogeneous isotropic material. The dependence of the refractive index on the intensity of the light wave leads to an additional, self-induced phase advance, $\phi_{N L}$, of the optical field when propagating over a distance $L$, which is given by [9]

$$
\phi_{\mathrm{NL}} \cong-n_{2} I(t) \omega_{0} \frac{L}{c}
$$


where $\omega_{0}$ is the angular carrier frequency of the optical pulse. Inspecting the instantaneous (time-dependent) angular frequency of the optical pulse which is defined as $\omega(t)=\omega_{0}+\delta \omega(t)$ with

$$
\delta \omega(t)=\frac{d \phi_{\mathrm{NL}}}{d t}
$$

shows that the Kerr effect leads to a self-induced phase and frequency change in light pulses. The leading edge of the optical pulse has an intensity increasing with time, which leads to a shift to lower frequencies (red shift) due to the minus sign in Eq. 2.26 (assuming that $n_{2}$ is positive, which is the case for most materials). The trailing edge of the optical pulse corresponds to a decrease in intensity with time which causes a shift to higher frequencies (blue shift). This Kerr-effect based nonlinear process is known as self-phase modulation (SPM) and leads to a spectral broadening of the optical pulse.

In order to compare the strength of the Kerr effect between waveguides made from different materials and different cross sections, a figure of merit, $\gamma$, is used. This figure of merit is commonly referred to as the nonlinear parameter, and is defined as [13]

$$
\gamma=\frac{n_{2} \omega}{A_{\mathrm{eff}} c}
$$

where $A_{\text {eff }}$ is the effective mode area as given by Eq. 2.9. The small values for the effective area that can be obtained in optical waveguides points towards a clear advantage of using waveguides for third-order nonlinear processes [13] . For comparison, typical values for $A_{\text {eff }}$ in standard single-mode fibers (low index contrast, $\Delta n \sim 10^{-3}$ ) are of the order of $20 \mu m^{2}$ [13] while for integrated $\mathrm{Si}_{3} \mathrm{~N}_{4} / \mathrm{SiO}_{2}$ waveguides $(\Delta n \sim 0.5)$ the area can assume values well below $1 \mu m^{2}$ [14].

For the description of propagation of a short pulse through a medium it is not sufficient to take only a single third-order effect into account but one has to consider also the linear dispersion. Specifically at high intensities one also has to take into account four wave mixing, and possibly Raman scattering, as well as the spatial, temporal and spectral interplay between all these effects.

The dispersion describes how an injected pulse tends to spread temporally due to the GVD and how it deforms the pulse. The result is a lowered peak intensity which decreases the nonlinear response of the medium. SPM describes how the pulse tends to spread spectrally. In the presence of normal dispersion, the increased bandwidth would lead to an increased temporal spreading which would lower the peak intensity of the pulse further. However, if the group velocity dispersion changes sign by providing anomalous dispersion, pulse spreading is turned into pulse compression. This increases the peak power after SPM has broadened the spectrum. If the optical pulse experiences the proper anomalous dispersion with the right intensity, the pulse can propagate through the waveguide with progressive spectral 
broadening and temporal shortening until reaching a constant temporal and spectral width. Such a pulse is called a fundamental soliton [10]. At increased intensity also higher-order solitons can be generated, which show an oscillatory spectral and temporal envelope. The presence of higher-order dispersion or Raman scattering eventually leads to the break-up of high-order solitons into various fundamental solitons [10].

Another third-order nonlinear optical process that can play a role during such nonlinear propagation of ultra-short optical pulses in dielectric waveguides is fourwave mixing (FWM), which is best explained in the spectral domain. In FWM, three optical fields at frequencies, $\omega_{1}, \omega_{2}$ and $\omega_{3}$ generate a fourth optical field at frequency $\omega$ via the third-order nonlinear polarization. For example, $\omega=\omega_{1}+\omega_{2}-\omega_{3}$, or $\omega=\omega_{1}-\omega_{2}+\omega_{3}$. These processes are similar to second-order nonlinear processes and differ only in the order of the susceptibility and the number of optical fields (four instead of three). The particular FWM process taking place again depends on phase-matching and the relative phase of the optical fields involved. SPM is a special case of FWM, where phase matching is fulfilled because the involve frequencies are all approximately the same.

The nonlinear process-based phenomenon that we mention finally is so called supercontinuum generation (SCG). This term designates the general observation that the propagation of a short optical pulse can result in spectral broadening to an extremely wide spectrum [10]. SCG usually comprises the complex interplay of dispersion with third-order processes as mentioned, with some additional but related effects. SCG starts with SPM and an initial broadening of the spectrum of the pulse. Together with proper anomalous dispersion and non-linear self-steepening this leads to the generation of ultra-short higher-order solitons with associated spectral broadening of the pulse. These solitons eventually break up in fundamental solitons due to the presence of high-order dispersion. The dispersion and broad spectrum associated with these pulses allows also for phase-matched FWM which coherently transfers energy from solitons to so-called dispersive waves at frequencies $\omega_{D W}$, that lie in the normal dispersion regime and that are determined by the following nonlinear FWM phase matching condition [10]

$$
\beta_{\omega_{s}}-\omega_{s} / \nu_{g, s}+\left(1-f_{R}\right) \gamma P_{s}=\beta\left(\omega_{D W}\right)-\omega_{D W} / \nu_{g, s} .
$$

In this expression, $\nu_{g, s}$ is the group velocity of generating soliton, $f_{R}$ is a correction for a possible Raman contribution, and $P_{s}$ the soliton peak power at frequency $\omega_{s}$. These dispersive waves usually form the outer spectral components in a wide supercontinuum spectrum.

Due to the large complexity of the described overall interaction and the strong dependence on specific material and design parameters, a simple analytical modeling of the output spectrum is not possible. A model that can yield a quite satisfying 
agreement with experimental observations is the so-called generalized nonlinear Schrödinger equation (GNLSE) [10], which we have applied as well for comparison with experimental spectral.

$$
\begin{array}{r}
\frac{\partial E}{\partial z}+\frac{\alpha}{2} E-\sum_{k \leq 2} \frac{i^{k+}}{k !} \beta_{k} \frac{\partial^{k} E}{\partial T^{k}}=i \gamma\left(1+i \tau_{s} \frac{\partial}{\partial T}\right) \\
\left(E(z, t) \int_{-\inf }^{+\inf } R\left(T^{\prime}\right) \times\left|E\left(z, T-T^{\prime}\right)\right|^{2} d T^{\prime}+i \Gamma_{R}(z, T)\right) .
\end{array}
$$

In this equation $E(z, t)$ is the time dependent field in the direction of propagation, $z, T=t-\beta_{1} z, \alpha$ describes the propagation losses. The dispersive coefficients $\beta_{k}$ are defined by Eq. 2.11. The field product in the second bracket on the right-hand side models the third-order nonlinear effects. The response function $R(t)$ provides the nonlinear response to a medium as an instantaneous electronic and a delayed $\left(f_{R}\right)$ Raman contribution, while the term $\Gamma_{R}$ models noise from spontaneous Raman emission or spontaneous FWM. The time scale indicated with the parameter $\tau_{s}$ models optical shock formation and self-steepening effects. The GNLSE was numerically solved using the split-step Fourier method [15].

\subsection{Coherent photogalvanic effect}

Centrosymmetric materials, specifically amorphous materials, do not possess an intrinsic bulk second-order nonlinearity [9]. Therefore, one would expect that $\mathrm{Si}_{3} \mathrm{~N}_{4}$ waveguides would not support second-order nonlinear optical processes excluding, e.g., the observation of SHG or the electro-optic effect. Fortunately, there are several physical processes that can break the material symmetry, and lead to the formation of a second-order nonlinearity. One way would be to apply a gradient stress to the waveguide [16]. Here, we discuss an all-optical method that is based on the third-order nonlinearity to generate a second-order nonlinear grating within the waveguide using a process, known as the coherent photogalvanic effect (CPGE). The effect has already been observed in glass fiber systems $[17,18]$ and is related to the photorefractive effect [9].

It should be noted that there are several phenomenological $[18,19]$ and theoretical $[18,20,21]$ models that describe the coherent photogalvanic effect. A more detailed description of the photogalvanic effect in the waveguides investigated will be presented in Chapter 5. In brief, the effect relies on the generation of free charges, based on multi-photon excitation, that are subsequently separated and trapped [18]. The named separation is based on third-order nonlinear optical rectification, which can induce a strong DC field distribution, resulting in the build up of a space-charge 
field distribution, until this balances the rectification field. The electric field associated with this charge separation provides the asymmetry required to create a second-order nonlinearity. If the displaced charges are immobilized in the dielectric, due to lack of conductivity and trapping to defects sites, the created charge separation is long-lived, i.e., remains present also after the inducing light field is turned off [22]. In the case of stoichiometric silicon nitride, silicon and nitrogen dangling bonds, as well as other defects (hydrogen and oxygen) play a role as trap sites [23].

If one assumes that initially some small SH field is present (e.g., from local spurious second-harmonic generation), then the third-order nonlinearity can generate a DC electric field pattern $\mathcal{E}_{\mathrm{DC}}$ via

$$
\mathcal{E}_{\mathrm{DC}}=\chi^{(3)}(\omega, \omega, 2 \omega ; 0=2 \omega-\omega-\omega) E(\omega) E(\omega) E(2 \omega)^{*} \exp (i \Delta \beta z)
$$

where $E(\omega)$ is the local field of the pump laser, $E(2 \omega)$ is the local field of the secondharmonic of the pump within the waveguide, $z$ is the direction of propagation, and $\Delta k=2 k(\omega)-k(2 \omega)$ is the wave vector mismatch. Due to the amorphous nature of $\mathrm{Si}_{3} \mathrm{~N}_{4} / \mathrm{SiO}_{2}$ waveguides and because all the electric fields have the same linear polarization, as a simplification we have taken the fields and $\chi^{(3)}$ in Eq 2.31 as scalars. The presence of photo-induced conduction charges generates a photocurrent, $j_{p h}$, which builds up a charge separation over time until that space-charge field balances the driving $\mathcal{E}_{\mathrm{DC}}$ field. Depending on the average power of the pump field, it may take up to several hours before equilibrium is reached (see Chapter 5). Important is that due to the phase mismatch between the field at the pump and secondharmonic wavelengths, the $\mathcal{E}_{\mathrm{DC}}$ field is generated with a spatial periodicity, $2 \pi / \Delta \beta$. Consequently, the second-order nonlinearity induced by the charge-field pattern shows the same spatial periodicity, which results in perfect quasi-phase matching for second-harmonic generation [24].

As an example, Fig. 2.6 shows the QPM period required to be generated via the photogalvanic effect to phase match a fundamental mode $\left(E_{11, y}\right)$ beam at $1064 \mathrm{~nm}$ and the different possible transverse modes of its second-harmonic for $y$-polarized light. In this example, it can be seen that for phase-matching the second-harmonic in the fundamental mode $\left(\mathrm{E}_{11, y}\right)$ as well, the required period is very small $(\approx 50 \mu \mathrm{m})$ for all shown waveguide widths, due to a large mismatch. However, for certain higher-order modes the required period for QPM is much larger, about several hundred micrometers $\left(\mathrm{E}_{12, y}\right.$ and $\left.\mathrm{E}_{31, y}\right)$. For two of the transverse modes, $\mathrm{E}_{12, y}$ and $\mathrm{E}_{31, y}$, even modal phase matching is possible near $0.45 \mu \mathrm{m}$, i.e., QPM is not required (infinite period in Fig. 2.6). For other waveguide widths at those modes, QPM is again required. 


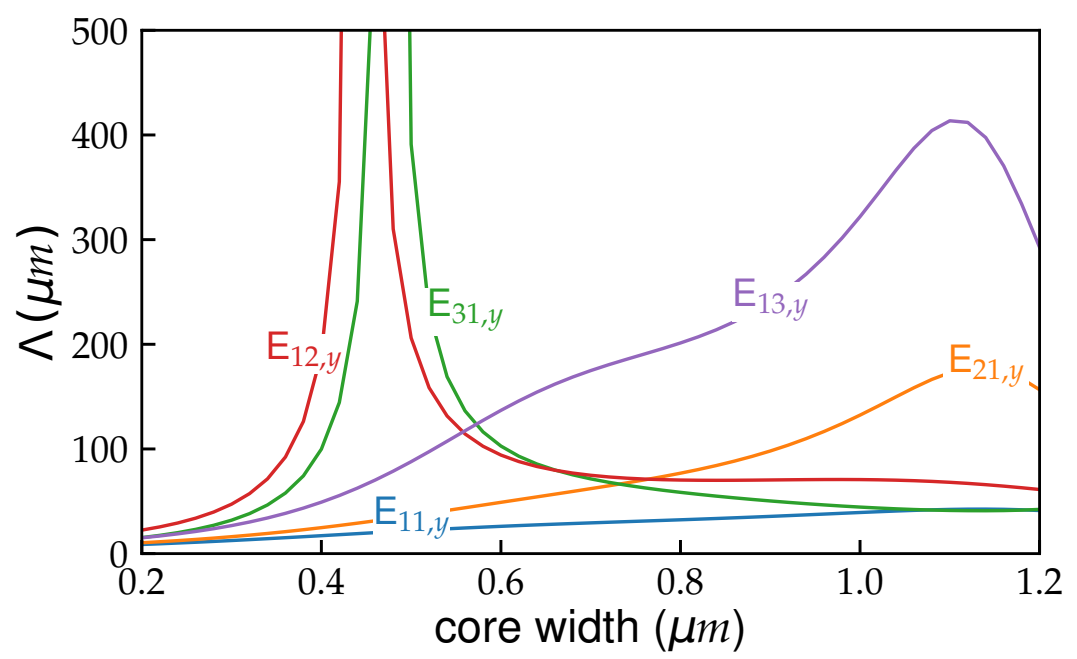

Figure 2.6: Quasi-phase matched period, $\Lambda$, as a function of the waveguide width of the core for various transverse modes at the second harmonic frequency and the fundamental mode at the fundamental frequency. All modes are polarized in the $y$-direction.

\subsection{Acousto-optic interaction: Surface acoustic waves}

So far, we have discussed nonlinear processes that solely involve optical fields. However, there are also nonlinear optical processes that rely on the so-called acoustooptic effect [9], where an acoustic wave influences the properties of an optical wave. Acousto-optic interactions find applications in many fields [25-28]. However, here we limit the discussion to a certain type of acoustic waves that are relevant for this work. In order to provide a maximum interaction with light in planar waveguide circuits that are located closely under the surfaces of PICs, we consider acoustic waves that deliver their maximum amplitude near the surface of the PICs.

Acoustic waves are typically generated using piezoelectric materials that allow fast and strong coupling between the driving electrical signal and the generated acoustic wave. However, these materials are generally not well suited as optical waveguides due to their large propagation losses. Although some piezoelectric materials, such as $\mathrm{LiNbO}_{3}$, exhibit also appropriate optical properties, the integration with photonic integrated circuits is difficult and tends to show undesired photorefractive effects at shorter wavelengths [29].

Therefore, we decided to follow an approach in which the guiding of the optical wave is separated from the generation of an acoustic wave. As the optical mode is 
confined to the neighborhood of the waveguide core and the acoustic waves propagates through solids, the optical waveguide can be placed close to a acoustic source material without, however, increasing the optical propagation loss. Among the different types of acoustic waves we will consider only Rayleigh surface acoustic waves (SAWs) because this type of acoustic wave is confined to a surface layer with a thickness of approximately an acoustic wavelength, and because it shows the lowest dispersion compared to other types of surface waves [26]. A low dispersion allows smaller pulse distortion when an acoustic pulses propagate through the material for obtaining a pulsed (instead of a periodic) modulation of light.

As the acoustic wavelength is much longer than the optical wavelength, a good overlap can be obtained between the surface acoustic wave and the optical wave, which is guided by a core that is buried just below the surface. This is schematically shown in Fig. 2.7, which shows a thin piezoelectric layer deposited above a a waveguide consisting of a $\mathrm{Si}_{3} \mathrm{~N}_{4}$ core surrounded by a $\mathrm{SiO}_{2}$ cladding. The core is located below the surface such that the optical field is practically zero inside the piezoelectric layer and the optical losses induced by this piezoelectric layer can be neglected, even for very long waveguides (with lengths of the order of many centimeter). A so-called interdigitized transducer electrode [26], or IDT, is placed on top of the piezoelectric layer to drive an surface acoustic wave with an acoustic wavelength, that is equal to the IDT period and that provides sufficient overlap with the optical wave.

In general, an acoustic wave induces strain (or stress) which results in displacement of volume elements in the material and, consequently, in a material deformation with an associated change in the density [25]. The strain and stress in a material are related by the constitutive equations, also known as Hooke's law,

$$
\sigma=\mathbf{c S},
$$

and its analogue

$$
\mathbf{S}=\mathbf{s} \sigma,
$$

where $\sigma$ and $\mathbf{S}$ are rank-two tensors for the stress and strain, respectively, while $\mathbf{c}$ and s are rank-four tensors for the stiffness, or elasticity, and the compliance, respectively [30].

The material deformation in an isotropic material is defined by the Newton equation [26],

$$
\nabla \cdot \mathbf{c} \mathbf{S}-\rho \frac{\partial^{2} \mathbf{u}}{\partial t^{2}}=0,
$$

where $\rho$ is the density of the material, and $\mathbf{u}$ is the material deformation. As the SAW travels, the local strain, $S$, becomes modulated and, therefore, the local refractive index of the material becomes modulated [25]. The strain tensor and the spatial deformation are related as $[25,30]$ 


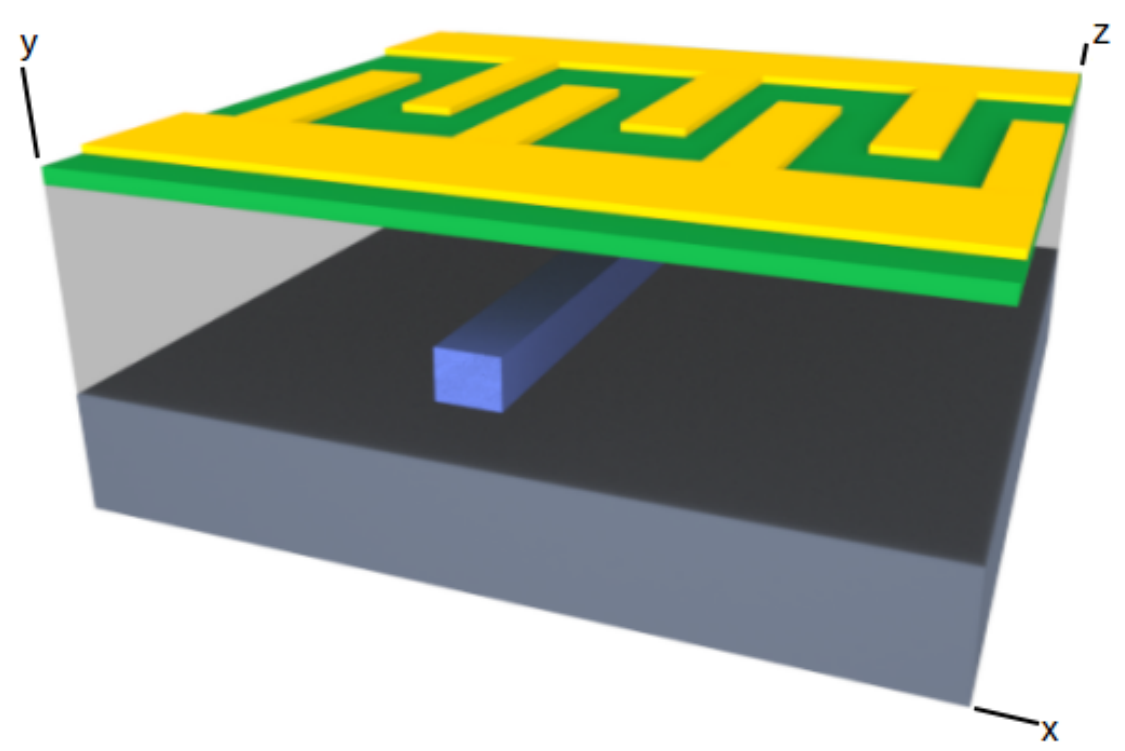

Figure 2.7: Schematic representation of an acousto-optic modulator based on surface acoustic waves. The electrodes (yellow) are located above the piezoelectric material (green) which is deposited over the waveguide cladding (light gray) and core (blue). The substrate (dark gray) is made out of silicon.

$$
S_{k j}=\frac{1}{2}\left(\frac{\partial u_{k}}{\partial x_{j}}+\frac{\partial u_{j}}{\partial x_{k}}\right),
$$

such that Eq. 2.34 actually represents a wave equation for $\mathbf{u}$.

A typical example of the strain distribution induced by the Rayleigh SAW generated using the configuration of Fig. 2.7 is displayed in Fig. 2.8. Shown are the strain distributions in the $x$-direction, $\left|S_{x}\right|$, normalized to its maximum value (a) and in the $y$-direction, $\left|S_{y}\right|$, normalized to its maximum. The shown region has a width equal to half of the IDT period, $\Lambda$, which corresponds to half of the acoustic wavelength, and a depth below the PZT layer of $3 / 2 \Lambda$. Each figure also contains the depth profile of the strain component containing the largest value of the strain (i.e., at $x=-\Lambda / 4$ ). The PZT layer extends from $y=0$ to a small depth where the $\mathrm{SiO}_{2}$ cladding begins. It can be seen in the figure that the strain has its maximum close to the surface, within a depth of $\Lambda / 2$, and decreases towards bigger depths. What can be seen as well from the antinode of $\left|S_{x}\right|$ in a certain depth is that $S_{x}$ changes its sign when the distance to the PZT layer increases.

In the case considered in this thesis, the materials are isotropic, so that their refractive index is uniform in every direction and, therefore, the refractive index tensor can be simplified to a scalar for a given wavelength. However, the acoustic strain 

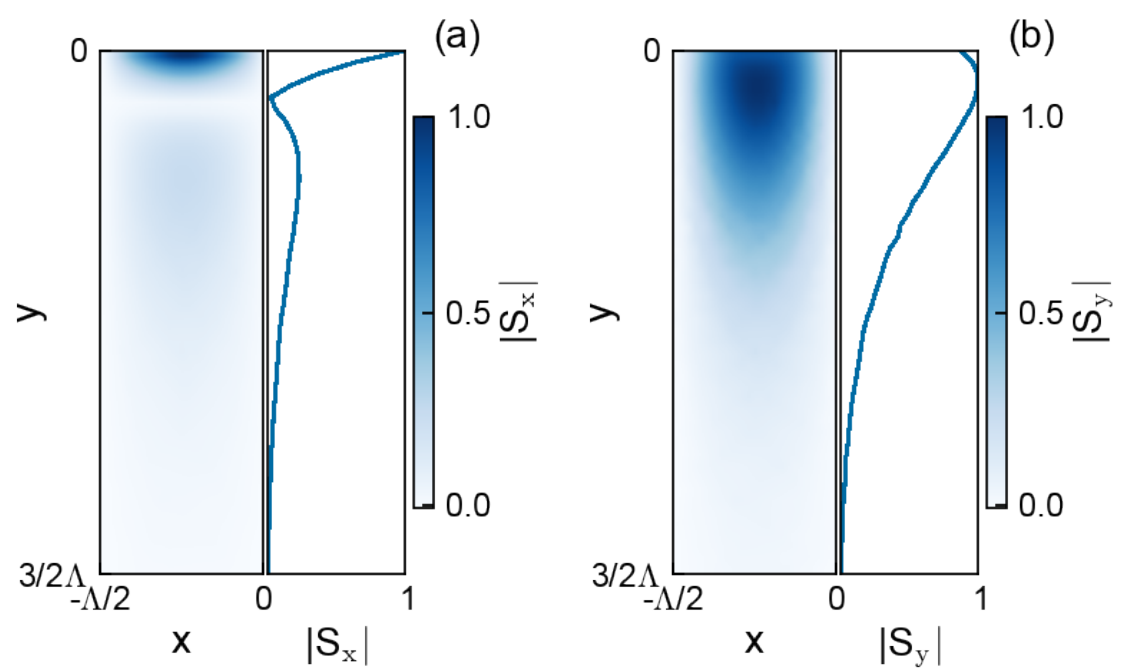

Figure 2.8: Strain distribution along the penetration depth of half a period of an acoustic wave being (a)-left the normalized horizontal strain, $\left|S_{x}\right|$, with its maximum depicted on the right, and (b)-left the vertical strain, $\left|S_{y}\right|$, with its maximum depicted on the right.

provides different changes in the refractive index depending on the direction of the strain. These index changes can be expressed as [25, 26, 28]

$$
\Delta\left(\frac{1}{n_{i d}^{2}}\right)=\sum_{j} \sum_{k} p_{i d k j} S_{k j}
$$

where $p_{i d k j}$ are the strain-optic coefficients, and $S_{k j}$ are the strain components. In the case of an isotropic material, the strain-optic coefficients can be compacted to a $6 \times 6$ matrix that has only two independent parameters, $p_{11}$ and $p_{12}$. The matrix elements are $p_{i j}=p_{12}$ and $p_{i i}=p_{11}$ for $i, j=1,2$, and 3 and $p_{i i}=1 / 2\left(p_{11}-p_{12}\right)$ for $i=4,5$, and 6 , with zero for all others elements $[25,30]$.

The interaction of the acoustic wave with the optical wave depends strongly on the propagation directions of both waves as well as the direction of the strain and the polarization of the optical wave. The two extreme cases are co-propagation, where both waves travel along a common axis and interaction occurs for several acoustic wavelengths, this configuration is used for acousto-optic long period grating modulators via mode conversion [28]. The second case is perpendicular propagation, which induces strain and index modulation across the entire optical length of the waveguide with the same phase.. Assuming the acoustic wave propagates in the $x$ direction and the optic wave in the $\mathrm{z}$-direction, there will be changes in the refractive 
index along the $x$-direction and the $y$-direction, as given by [25]:

$$
\Delta n_{i}=-\frac{1}{2} n^{3}\left(p_{i i} s_{i i}+p_{i j} s_{j j}\right)
$$

where $i=x$ and $j=y$, and vice versa. This change in the local refractive index changes the effective refractive index of the optical wave mode. Calculating the change in effective refractive index can be used to estimate the effectiveness of using SAW to implement various functionalities for the $\mathrm{Si}_{3} \mathrm{~N}_{4}$ photonic platform. The example of intensity modulation via index modulation in a Mach-Zehnder interferometer [25-28], is described in Chapter 4.

\section{References}

1. Saleh, B. E. A. \& Teich, M. C. Fundamentals of Photonics (Wiley, 2007).

2. Marcuse, D. Theory of Dielectric Optical Waveguides (Elsevier, 2013).

3. Maxwell, J. C. A Dynamical Theory of the Electromagnetic Field. Phil. Trans. R. Soc. Lond. 155, 459-512 (1865).

4. Marcatili, E. A. Dielectric rectangular waveguide and directional coupler for integrated optics. Bell Syst. Tech. J. 48, 2071-2102 (1969).

5. Coggon, J. H. Electromagnetic and Electrical Modeling by the Finite Element Method. Geophys. 36, 132-155 (1971).

6. Fallahkhair, A. B., Li, K. S. \& Murphy, T. E. Vector Finite Difference Modesolver for Anisotropic Dielectric Waveguides. J. Lightwave Technol. 26, 1423-1431 (2008).

7. Luke, K., Okawachi, Y., Lamont, M. R. E., Gaeta, A. L. \& Lipson, M. Broadband midinfrared frequency comb generation in a $\mathrm{Si}_{3} \mathrm{~N}_{4}$ microresonator. Opt. Lett. 40, 4823-4826 (2015).

8. Malitson, I. H. Interspecimen Comparison of the Refractive Index of Fused Silica*,. J. Opt. Soc. Am. 55, 1205-1209 (1965).

9. Boyd, R. W. Nonlinear Optics (Academic Press, 2013).

10. Dudley, J. M., Genty, G. \& Coen, S. Supercontinuum generation in photonic crystal fiber. Rev. Mod. Phys. 78, 1135-1184 (2006).

11. Suhara, T. \& Fujimura, M. Waveguide Nonlinear-Optic Devices red. by Kamiya, T., Monemar, B., Venghaus, H. \& Yamamoto, Y. (Springer Berlin Heidelberg, Berlin, Heidelberg, 2003).

12. Fejer, M. M., Magel, G. A., Jundt, D. H. \& Byer, R. L. Quasi-phase-matched second harmonic generation: tuning and tolerances. IEEE J. Quant. Electron. 28, 2631-2654 (1992).

13. Agrawal, G. P. Nonlinear Fiber Optics (Academic Press, 2007).

14. Epping, J. P. Dispersion engineering silicon nitride for broadband nonlinear frequency conversion $\mathrm{PhD}$ thesis (2015).

15. Taha, T. R. \& Ablowitz, M. I. Analytical and numerical aspects of certain nonlinear evolution equations. II. Numerical, nonlinear Schrödinger equation. J. Comp. Phys. 55, 203-230 (1984). 
16. Khurgin, J. B., Stievater, T. H., Pruessner, M. W. \& Rabinovich, W. S. On the origin of the second-order nonlinearity in strained SiSiN structures. J. Opt. Soc. Am. B 32, 2494 (2015).

17. Margulis, W. \& Österberg, U. Second-harmonic generation in optical glass fibers. J. Opt. Soc. Am. B 5, 312-316 (1988).

18. Anderson, D. Z., Mizrahi, V. \& Sipe, J. E. Model for second-harmonic generation in glass optical fibers based on asymmetric photoelectron emission from defect sites. Opt. Lett. 16, 796-798 (1991).

19. Sokolov, V. O. \& Sulimov, V. B. On the phenomenological theory of the coherent photogalvanic effect in glass. Phys. Status Solidi B 187, 177-187 (1995).

20. Sulimov, V. B. Theory of the coherent photovoltaic effect and the method of nonequilibrium Green's functions. J. Exp. Theor. Phys. 101, 1749-1771 (1992).

21. Sokolov, V. O. \& Sulimov, V. B. Theory of third-order nonlinear photoconductivity and third-order photogalvanic effect in glass. Phys. Status Solidi B 187, 189-204 (1995).

22. Warren, W. L., Rong, F. C., Poindexter, E. H., Gerardi, G. J. \& Kanicki, J. Structural identification of the silicon and nitrogen danglingbond centers in amorphous silicon nitride. J. Appl. Phys. 70, 346-354 (1991).

23. Robertson, J., Warren, W. L. \& Kanicki, J. Nature of the Si and N dangling bonds in silicon nitride. Journal of Non-Crystalline Solids. Amorphous Insulating Thin Films II 187, 297-300 (1995).

24. Dianov, E. M. \& Starodubov, D. S. Photoinduced generation of the second harmonic in centrosymmetric media. Quantum Electron. 25, 395-407 (1995).

25. Korpel, A. Acousto-Optics, Second Edition (CRC Press, 1996).

26. Hashimoto, K.-Y. Surface Acoustic Wave Devices in Telecommunications: Modelling and Simulation (Springer Science \& Business Media, 2000).

27. Lima, M. M. d. \& Santos, P. V. Modulation of photonic structures by surface acoustic waves. Reports on Progress in Physics 68, 1639-1701 (2005).

28. Tsai, C. S. Guided-Wave Acousto-Optics: Interactions, Devices, and Applications (Springer Science \& Business Media, 2013).

29. Günter, P. Holography, coherent light amplification and optical phase conjugation with photorefractive materials. Phys. Rep. 93, 199-299 (1982).

30. Yariv, A. Quantum electronics (Wiley, 1989). 


\section{Chapter 3}

\section{Supercontinuum generation}

We demonstrate supercontinuum generation in stoichiometric silicon nitride $\left(\mathrm{Si}_{3} \mathrm{~N}_{4}\right.$ in $\left.\mathrm{SiO}_{2}\right)$ integrated optical waveguides, pumped at telecommunication wavelengths. The pump laser is a mode-locked erbium fiber laser at a wavelength of $1.56 \mu \mathrm{m}$ with a pulse duration of $120 \mathrm{fs}$. With a waveguide-internal pulse energy of $1.4 \mathrm{~nJ}$ and a waveguide with $1.0 \mu \mathrm{m} \times 0.9 \mu \mathrm{m}$ cross section, designed for anomalous dispersion across the $1500 \mathrm{~nm}$ telecommunication range, the output spectrum extends from the visible, at around $526 \mathrm{~nm}$, up to the midinfrared, at least $2.6 \mu \mathrm{m}$, the instrumental limit of our detection. This output expands more than 2.2 octaves $(454 \mathrm{THz}$ at the $-30 \mathrm{~dB}$ level). The measured output spectra agree well with the theoretical modeling based on the generalized nonlinear Schrödinger equation. The infrared part of the supercontinuum spectra shifts progressively towards the mid-infrared, well beyond $2.6 \mu \mathrm{m}$, by increasing the with of the waveguides. 


\subsection{Introduction}

Supercontinuum generation (SCG), typically obtained with femtosecond pulses in photonic crystal fibers [1], is a powerful method of providing extremely broadband spectra with full spatial and temporal coherence $[2,3]$. Such nonlinear optical generation is of high interest for numerous applications, for instance in spectroscopy [4], with visible wavelengths in the life sciences [5], or in precision metrology [2,6]. In addition, there is a growing relevance for coherent on-chip generation of broadband light based on lasers in the telecommunication wavelength range [7]. Envisioned applications include chip-sized frequency combs [8] for coherent transfer of terabit data streams [9] or wideband integrated microwave photonics [10,11].

Using standard photonic crystal fibers, which typically requires interaction lengths of tens of centimeters for efficient SCG, provides considerable design flexibility in their dispersive and nonlinear properties [1]. However, the shot-to-shot coherence of the SC output decreases with increasing pump pulse duration as well as with interaction length [1, 12], particularly in the presence of Raman scattering [12]. In contrast, the conversion of ultra-short pulses ( $\sim 100 \mathrm{fs})$ remains coherent in short fibers, but the conversion remains inefficient with a short interaction length. Employing integrated optical waveguides on a chip provides the advantage of tight mode confinement which strongly increases the nonlinear coefficient. This enables an efficient conversion of ultrashort pulses also with short interaction lengths while preserving coherence. For instance, pulses with up to a few hundred femtoseconds have been used for efficient SCG in short optical waveguides while maintaining a high degree of coherence [13-15]. As a second advantage the integrated optical approach offers a rout for high-volume and low-cost fabrication, in particular when the waveguide platform is compatible with metal-oxide-semiconductor (CMOS) fabrication facilities.

Lasers in the telecommunication range, due to their wide availability and maturity, have been employed for SCG in a variety of photonic platforms that are compatible with CMOS fabrication facilities. Examples are doped silica ridge waveguides using ultrashort pulses near $1300 \mathrm{~nm}$ and near $1550 \mathrm{~nm} \mathrm{[16,17],} \mathrm{buried} \mathrm{silicon} \mathrm{nitride}$ waveguides using pulses near $1300 \mathrm{~nm}$ [18], or silicon oxynitride waveguides with $1500 \mathrm{~nm}$ pulses [19]. The strongest nonlinear parameter and thus SCG requiring low pulse energies can be found in smaller-bandgap or adjustable bandgap materials, e.g. chalcogenides [20,21], silicon [22-24], and Si-enriched nitride glass [25]. Increasing the nonlinearity via reducing the bandgap is, however, associated with increased nonlinear losses at shorter wavelengths, such as via two-photon absorption.

A waveguide platform of highest relevance is stoichiometric $\operatorname{SiN}\left(\mathrm{Si}_{3} \mathrm{~N}_{4}\right)$ grown with low pressure chemical vapor deposition (LPCVD), because here a large variety of 
additional functionalities is available with wafer scale fabrication. These functionalities are based on the accessibility of an extremely wide wavelength range (from the blue, across the visible into the infrared) and that, providing different types of waveguide cross sections [26] and tapers [27], enable, e.g., polarization control, efficient fiber coupling, or bandwidth narrowing with hybrid-integrated diode lasers [28, 29]. The intrinsic absorption and scattering is weak [30] which offers exceptionally low propagation loss, below $0.001 \mathrm{~dB} / \mathrm{cm}$ for standard waveguides [31]. The latter is central for narrowband spectral filtering and optical delay lines as in quantum optical systems [32], in microwave photonic filters [33, 34] or in programmable optical processors [35]. Finally, as $\mathrm{Si}_{3} \mathrm{~N}_{4}$ has negligible Raman gain, highly coherent supercontinua can be generated using low pump energies [36]. So far in this platform SCG has been mainly investigated with a relatively short pump wavelength around $1 \mu \mathrm{m}$, yielding spectral coverage mainly at shorter wavelengths from the blue and across the visible into the near infrared [36-39].

Here, we demonstrate a significant spectral widening of SCG towards the midinfrared range. This was achieved with a longer pump wavelength in the $1.5 \mu \mathrm{m}$ telecommunication range in combination with dispersion engineering based on appropriate cross sections for the fabricated waveguides. These were designed to shift anomalous dispersion towards longer wavelengths, including the $1.5 \mu \mathrm{m}$ telecommunication wavelength range. The output spectra extend from about $526 \mathrm{~nm}$ to well beyond $2.6 \mu \mathrm{m}$, the limit of our detection instruments, thereby spanning at least 2.2 octaves, which is more than $454 \mathrm{THz}$ at the $-30 \mathrm{~dB}$ level. The spectra broaden towards the mid-infrared with an increasing width of the waveguides, in agreement with modeling.

\subsection{Experimental Setup}

For enabling SCG with the available pump wavelength of $1560 \mathrm{~nm}$, appropriate dimensions of the waveguide core have to be chosen such that anomalous dispersion, i.e., negative group velocity dispersion (GVD), is imposed broadly around that wavelength. Anomalous dispersion is characterized by a positive value of the dispersion coefficient, $D(\lambda)=-\lambda / c \cdot n^{\prime \prime}(\lambda)$ [40], where $\lambda$ is the vacuum wavelength, $c$ is the speed of light, and $n^{\prime \prime}(\lambda)$ is the second derivative of the effective refractive index of the propagating mode with respect to the wavelength. We used a full vectorial finite-element solver (Fimmwave, Photon Design) to calculate the effective refractive index $n(\lambda)$ as function of wavelength and then derived $D(\lambda)$. The twodimensional step-index profile used for modeling was based on the actual, somewhat rounded shape of the waveguide core as resulting from the fabrication process and obtained from scanning electron microscope images of the waveguide cross 
section [37, 41]. The material dispersion of stoichiometric silicon nitride and silica is given by Eqs. 2.7 and 2.8, respectively.

Figure 3.1 shows the calculated dispersion coefficient $v$ s. the wavelength for waveguides of various different widths between 0.7 and $1.3 \mu \mathrm{m}$, having the same height of $0.9 \mu \mathrm{m}$. The calculations imply that anomalous dispersion $(D>0)$ can be obtained over wide spectral ranges including the pump wavelength at $1560 \mathrm{~nm}$ if the waveguide core is chosen wider than approximately $0.8 \mu \mathrm{m}$. The calculations also show that increasing the waveguide width shifts the range of anomalous dispersion noticeably towards the mid-infrared range, which is expected to shift SCG to longer wavelengths as well.

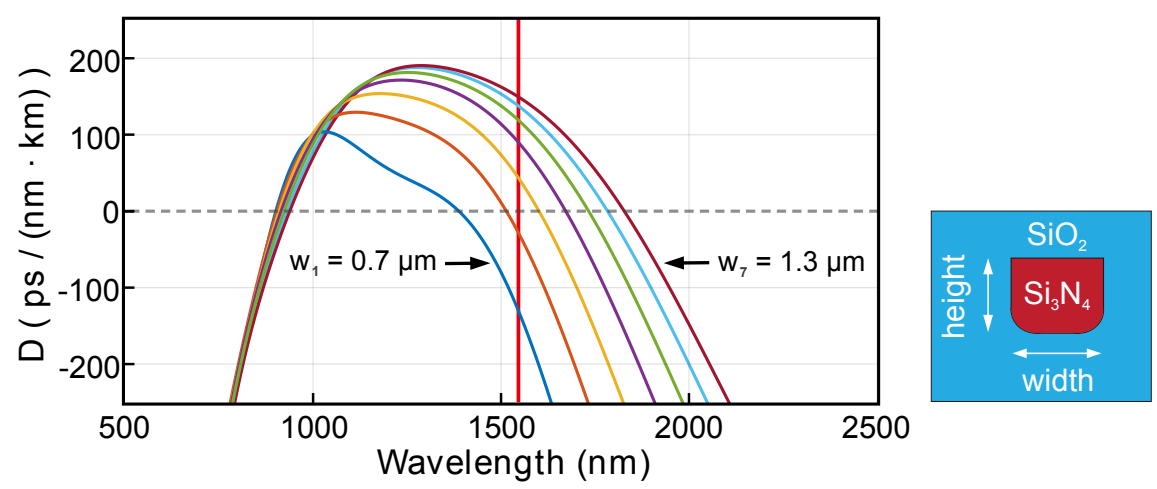

Figure 3.1: Calculated dispersion coefficient, $D(\lambda)$, for the fundamental TM-mode in $\mathrm{Si}_{3} \mathrm{~N}_{4}$ waveguides with a height $h=0.90 \mu \mathrm{m}$ and widths varied from $w_{1}=0.70 \mu \mathrm{m}$ to $w_{7}=1.3 \mu \mathrm{m}$ in steps of $0.1 \mu \mathrm{m}$. Anomalous dispersion is present above the dashed zero-dispersion line. The center wavelength of the pump laser at $1560 \mathrm{~nm}$ is indicated as red vertical line.

Figure. 3.2 shows the experimental setup used for SCG based on a standard modelocked Erbium fiber laser (Toptica, FemtoFiber Pro). The laser provided ultrashort pulses at a center wavelength of $1560 \mathrm{~nm}$, a repetition rate of $40 \mathrm{MHz}$, and a maximum average output power of $300 \mathrm{~mW}$ (maximum pulse energy $7.5 \mathrm{~nJ}$ ). The pulse duration was about 120 fs (full width at half maximum, FWHM), with a timebandwidth product of 0.75 , as measured with a home-made frequency resolved optical gating (FROG) autocorrelator. A combination of two half-wave plates (HWP) and a polarizing beam splitter (PBS) was used to control the power and the polarization of the beam. Two silver mirrors and an aspheric lens ( $N A=0.55$ at $1550 \mathrm{~nm})$ were used to steer and focus the beam into the waveguides, respectively. Several $\mathrm{Si}_{3} \mathrm{~N}_{4}$ chips were available as diced with a wafer saw, each chip carrying a set of 100 waveguides with various widths at a pitch of $20 \mu \mathrm{m}$ and a fixed length of $6 \mathrm{~mm}$. 


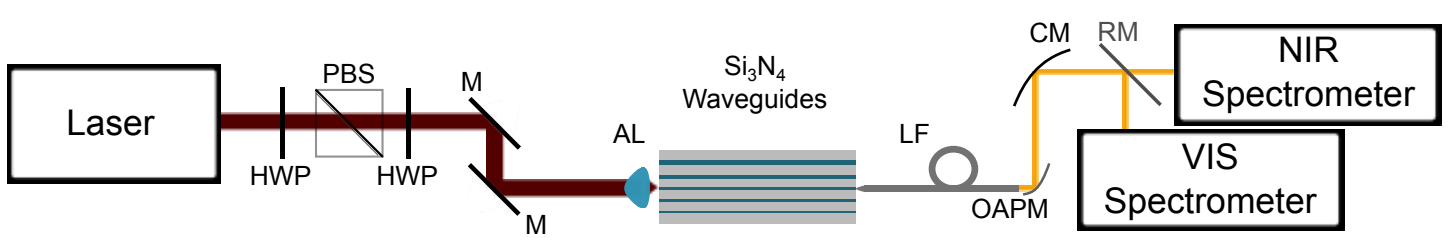

Figure 3.2: Schematic view of the experimental setup for supercontinuum generation (SCG) with ultrashort pulses from a mode-locked Erbium fiber laser. The pulses enter the $\mathrm{Si}_{3} \mathrm{~N}_{4}$-waveguide sample via two half-wave plates (HWP), a polarizing beam splitter (PBS) and an aspherical lens (AL). A lensed fiber (LF) and an off-axis parabolic mirror $(\mathrm{OAPM})$ collect and collimate the generated SC. A curved mirror $(\mathrm{CM})$ focuses the beam via a removable mirror (RM) into two different optical spectrum analyzers.

In order to determine the waveguide-internal power, the incident average power was measured in front of the first lens and the transmitted power was measured behind a second aspheric lens $(N A=0.55)$ that collected the radiation behind the waveguide. A low input power was used to avoid spectral broadening. Also, to ensure that only light exiting from the waveguide was measured, and not any light scattered or guided between the $\mathrm{SiO}_{2}$ cladding and Si bulk, the measurements were performed in the well-collimated part of the output beam, at a distance of about a meter behind the second lens. After optimizing the input and output coupling, typically $-8.8 \mathrm{~dB} \pm 0.6 \mathrm{~dB}$ of the incident power was transmitted through the waveguides. To determine the input coupling loss for each of the differently wide waveguides used, we subtracted from each measured transmission value the waveguideinternal propagation loss and output coupling loss. We have taken the propagation loss of the waveguides to be in the order of $-0.5 \mathrm{~dB} / \mathrm{cm}$ as based on previous measurements [41]. This corresponds to a total propagation loss of $-0.3 \mathrm{~dB}$ for the $0.6 \mathrm{~cm}$ long waveguide used. As output coupling loss we took the Fresnel loss at the output facet $(-0.4 \mathrm{~dB})$ and the truncation loss at the second aspheric lens $(-2.6 \mathrm{~dB})$ calculated from the almost Gaussian mode field emerging from the waveguide and the aperture of the lens. As a typical example, with the $1 \mu \mathrm{m}$ wide waveguide, where a transmission of $-8.7 \mathrm{~dB}$ was measured, we obtain an input coupling loss of $-5.4 \mathrm{~dB}$ which corresponds to an input coupling of about $29 \%$ of the incident power. This value can likely be increased with improved mode matching.

After the power throughput measurements, in order to conveniently record the spectral distribution of SCG, the second lens was removed and replaced with a lensed fiber. The fiber output was collimated with an off-axis parabolic mirror and focused by a curved mirror via a removable mirror into two optical spectrum analyzers (OSAs) operated in parallel. For the shorter wavelengths, from $380 \mathrm{~nm}$ to $1680 \mathrm{~nm}$, an OSA was used with a high dynamic range due to a low dark current 
(AQ1425 from ANDO, resolution $2 \mathrm{~nm}$ ). For longer wavelengths, between $1200 \mathrm{~nm}$ and $2584 \mathrm{~nm}$, a second OSA was available (NIRQuest 512 from OceanOptics, resolution $6.3 \mathrm{~nm}$ ). The latter makes use of a CCD-array and thus shows a higher level of dark counts that was subtracted from the recorded spectra. The recordings with the first OSA did not require subtraction. Within their overlap range the spectra of the two OSAs displayed the same shape. We used the agreement to merge the OSA spectra, at a wavelength of $1550 \mathrm{~nm}$, such that each supercontinuum spectrum is displayed as a single curve. To enable a direct comparison, all spectra were normalized to their peak values. Employing waveguides with seven different widths and the same height $(0.9 \mu \mathrm{m})$ we measured the spectral power density of the generated output $v s$. the pump pulse energy incident on the aspheric lens in a range between 0.3 and $6.8 \mathrm{~nJ}$, which corresponds to about $100 \mathrm{pJ}$ to $2 \mathrm{~nJ}$ of waveguide-internal pulse energy.

\subsection{Experimental Results}

Figure 3.3(a) shows a series of measured spectra as obtained with a $1 \mu \mathrm{m}$ wide waveguide. This particular example is shown here because all of the main spectral features still lie within the spectral range observable with our pair of OSAs.

It can be seen that the recorded spectra display all well-known features that are typical for SCG as described to large detail, e.g., in Ref. [1]. At the lowest recorded energy the output spectrum already starts to deviate from the incident pump laser spectrum (as retrieved from FROG traces), as is apparent from the two peaks induced by self-phase modulation at 1520 and $1590 \mathrm{~nm}$. The asymmetry in the spectral power density of the two peaks is caused by a slight spectral asymmetry in the pump spectrum. With increasing incident pulse energy, up to $1.8 \mathrm{~nJ}$, there is a spectrally symmetric, steady and steepening increase in bandwidth which indicates increasing self-phase modulation in the waveguide. Approximately at $2.3 \mathrm{~nJ}$, the spectrum reaches almost its maximum bandwidth extending from about $515 \mathrm{~nm}$ to $2250 \mathrm{~nm}$ at a $-30 \mathrm{~dB}$ level. The central part of the spectrum, between 1170 and $1750 \mathrm{~nm}$ at $-20 \mathrm{~dB}$ level, can be addressed to soliton dynamics because its extent agrees well with the expected range of anomalous dispersion (900 to $1700 \mathrm{~nm}$ as seen in Fig. 3.1) required for soliton formation. Such dynamics are also apparent from an increasing number of finer spectral features that separate and shift away from the pump wavelength with increasing energy, indicating fission into lowerorder solitons. Starting with $1.8 \mathrm{~nJ}$, at the outer edges of the spectrum there appear additional features with narrower bandwidths located at about $520 \mathrm{~nm}$, and located in the wavelength region from approximately $2050 \mathrm{~nm}$ to $2270 \mathrm{~nm}$. These features reveal the generation of dispersive waves by solitons (Cherenkov radiation), due to their smaller spectral width and because their wavelengths lie fully within the 


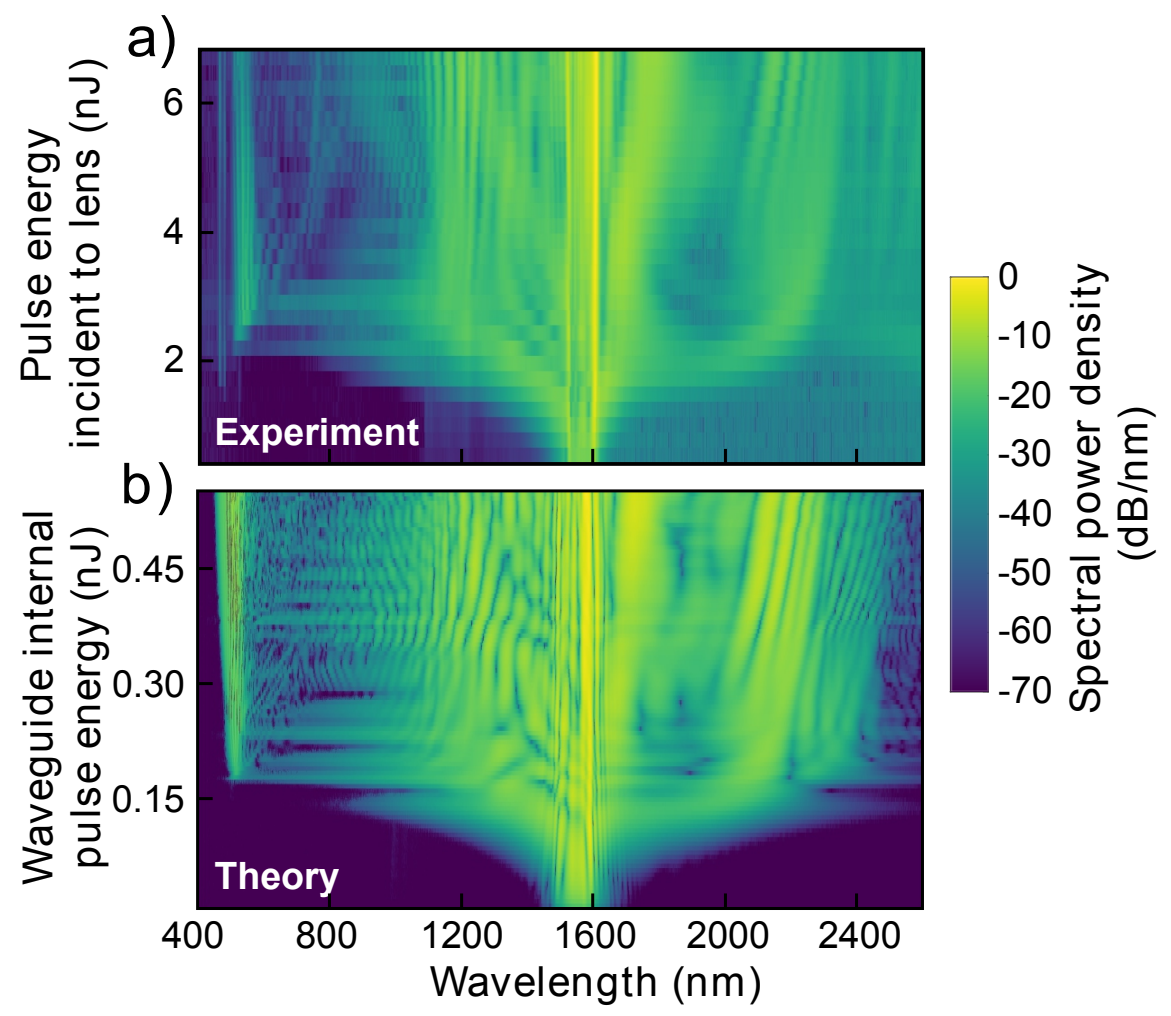

Figure 3.3: a) Measured spectral power density (color-coded) obtained from supercontinuum generation in a $0.9 \mu \mathrm{m}$ high and $1 \mu \mathrm{m}$ wide $\mathrm{Si}_{3} \mathrm{~N}_{4}$ waveguide, using ultrashort pulses (120 fs) from an Erbium fiber laser at $1560 \mathrm{~nm}$. The spectra are displayed vs. the pump pulse energy incident on the aspheric lens (upper vertical axis), of which about $30 \%$ was coupled into the waveguide. Each spectrum is normalized to its peak value. b) Theoretical spectral density $v s$. waveguide internal pulse energy (lower vertical axis) as obtained from simulations with the experimental values of the waveguide parameters. 
range of normal dispersion $(\mathrm{D}<0)$. At further increased energy of the pump pulses, beyond about $3.5 \mathrm{~nJ}$, the increase in spectral bandwidth with pulse energy is weaker and can be addressed mainly due to a Kerr-induced red-shift of phase matching for the long-wavelength dispersive wave to about $2300 \mathrm{~nm}$, as we find little to no shift in the short-wavelength dispersive wave at about $520 \mathrm{~nm}$.

\subsection{Numerical Simulations}

For comparing the measured spectra with calculations, we modeled the pulse propagation with the generalized nonlinear Schrödinger equation (GNLSE) using the split-step Fourier transform method $[42,43]$ as implemented by Kues et al. [44]. The motivation is to determine and verify input parameters that properly calibrate the model. This is important because the nonlinear optical properties of waveguides fabricated with the present material platform have not been fully characterized.

Specifically, there is little information on the Kerr index of LPCVD-grown $\mathrm{Si}_{3} \mathrm{~N}_{4}$. The only information available so far has been given by Levy [45] who derived the Kerr index from measuring the spectral broadening induced by self phase modulation. He reported a value of $(1.1-1.2) \times 10^{-15} \mathrm{~cm}^{2}$

$\mathrm{W}^{-1}$. Another value, which is often used in simulations, has been reported by Ikeda et al., $2.4 \times 10^{-15} \mathrm{~cm}^{2} \mathrm{~W}^{-1}$ [46], albeit for a different fabricated material, i.e., using a lower-temperature, plasma enhanced growth technique (PECVD). Another concern is that in [46], Ikeda et al. did not specify the Si-to-N atomic ratio, leaving open whether the material was stoichiometric. These dissimilarities are important because PECVD-grown silicon exhibits an increased absorption at around $1.5 \mu \mathrm{m}$ wavelength, where also the Kerr-measurements were performed. Other dissimilarities are a higher pinhole density and a higher hydrogen content [47]. These properties not only lead to higher loss and scattering but should also induce a higher nonlinearity as compared to LPCVD material. Specifically deviations from stoichiometric growth can strongly increase the nonlinearity as found for enrichment with Si [48].

For definiteness in the simulations and for retrieving more information on the Kerr index of LPCVD-grown $\mathrm{Si}_{3} \mathrm{~N}_{4}$ via comparison with experimental spectra, we preliminary used the Kerr index given by Ikeda et al. [46]. The size of the effective modal area, $A_{\text {eff }}$, was calculated with the finite element solver along with the waveguide dispersion as described above which results in a nonlinear coefficient $\gamma$ of $1.01 \mathrm{~W}^{-1} \mathrm{~m}^{-1}$. The dispersion was included to high precision, up to the 19 th order. A contribution from stimulated Raman emission to the nonlinearity was neglected, in view of previous experiments with SCG in silicon nitride waveguides [18, 49]. A 
shock term was included to model the frequency dependence of the nonlinear index and of the effective modal area [50]. For the $1.0 \mu \mathrm{m} \times 0.9 \mu \mathrm{m}$-sized waveguides, e.g., we calculated a shock time scale of 1.9 fs. The propagation loss was taken to be $0.5 \mathrm{~dB} / \mathrm{cm}$ [41]. As the input pulse we took the experimental power and phase spectrum as measured with FROG. We note, that our modeling does not rely on any adjustable fit parameters. This is different from, e.g., [19], where the waveguide dimensions were adjusted to fit the simulations to experimental spectra.

Figure 3.3(b) displays a series of 100 calculated supercontinuum spectra vs. increasing waveguide-internal pump pulse energy, assuming the same waveguide width $(1 \mu \mathrm{m})$ and height $(0.9 \mu \mathrm{m})$ as used for the measurements. It can be seen that there is a very good agreement of the spectral distribution with the experimental data. One can recognize essentially the same spectral development $v$ s. increasing pulse energy, from self-phase modulation towards soliton formation and fission. The agreement suggests that the characteristic length of nonlinear interaction, the dispersion length (strength of anomalous dispersion) and detailed shape of waveguide dispersion contained in higher order terms are appropriately described within the anomalous range. We note that also the spectral positions of the long and short-wavelength dispersive waves are predicted with high agreement, with only a small offset for the low-wavelength edge of the SC. This discrepancy is attributed to a small difference in material dispersion at short wavelengths. This implies that phase matching was modeled with proper dispersion in the outer, normal dispersion range (especially for the longer wavelengths), including a proper group velocity of the solitons that excite dispersive waves. Finally, also the spectral Kerr shift of the dispersive waves, here mainly at the long-wavelength side, is matching well the experiments. The overall agreement, specifically the absence of self-frequency shifting, confirms that neglecting stimulated Raman scattering is justified here. We note that the absence of Raman contributions is a main advantage as compared to SC generation in optical fibers where such non-parametric contribution may introduce noticeable shotto-shot incoherent output. Furthermore, from the absence of Raman scattering a coherent supercontinuum is expected [36]. We have confirmed coherent generation explicitly with calculation of the shot-to-shot coherence for the supercontinua presented in Fig. 3.3. We found a near unity degree of coherence for the entire spectral range that has a larger than $-30 \mathrm{~dB}$ relative spectral power density. This result is in agreement with the calculation reported by Epping et al. [37] where neglecting Raman scattering was also justified and where pumping at $1 \mu \mathrm{m}$ wavelength showed near unity pulse-to-pulse coherence as well [51]. By spectrally integrating the output and comparing with the internal pump pulse energy we observe that the calculated waveguide-internal conversion efficiency is very high, typically about $93 \%$, due to the absence of Raman conversion and due to low waveguide propagation loss.

As the next step, based on high agreement between the simulated measurements 
spectral distributions, we also compare the waveguide-internal pulse energies required for SCG in the experiment with those predicted by the calculations. As described above, we have concluded from throughput measurements and loss estimates that typically $29 \%$ of the incident power is coupled into the waveguide (input coupling loss $-5.4+/-0.6 \mathrm{~dB}$ ). For an unambiguous comparison of pump energies we chose the onset of the dispersive wave at the shorter wavelength side of the spectrum. In the experiment the dispersive waves occurs at around $2.1 \mathrm{~nJ}$ of incident energy, corresponding to about $0.61 \mathrm{~nJ}$ internal energy. Compared to this, in the simulations the dispersive wave generation sets in already at $0.17 \mathrm{~nJ}$, which is a factor 3.6 lower.

In order to identify possible reasons for this deviation we inspected several effects. A relatively large uncertainty is usually present regarding the input coupling loss. Here, however, transmission measurements provide a lower bound for these losses. Underestimating losses in the waveguide, output coupling or aperturing at the second lens would rather increase the estimated value for input coupling. A slight misalignment of the input coupling with increasing pump power was observed. We conclude this from a slightly sub-linear growth of the wavelength-integrated OSA spectra vs. incident pump power, spanning from the low-power values used for transmission experiments towards the higher-power for SCG . However, these measurements limit an underestimation of the input coupling to a factor of 1.3 at most. Another source of error might have been pump energy coupled into higher-order modes, which have a different dispersion and, therefore, do not contribute to the SCG. However, input coupling to higher-order modes has been experimentally investigated with great care showing that such coupling can safely be neglected for our experimental conditions. Similarly, measuring the transmitted power in the collimated beam far behind the output lens rules out that the transmission measurements are impaired by light which was not coupled into the waveguide but still scattered or guided in the $\mathrm{SiO}_{2}$ cladding towards the output facet. In the model, a source of error might have been an underestimated size of the mode field area, via underestimating the waveguide cross sectional area, which overestimates the nonlinear coefficient. However, the very good spectral agreement of the calculated and measured spectra indicate that the waveguide dispersion was modeled correctly, which rules out a wrongly assumed waveguide cross section. Our overall conclusion from these considerations is that the nonlinear refractive index used in the simulations is larger than in the waveguides that we have experimentally investigated. Quantitatively, the nonlinear refractive index for our waveguides appears to be about 3.6-times smaller than the previously reported value for PECVD-grown silicon nitride [46] and about 1.8-times smaller than the value reported for LPCVDgrown $\mathrm{Si}_{3} \mathrm{~N}_{4}$, which is obtained from SPM-induced spectral broadening [45]. A further clarification might be obtained by repeating the power modulation experiments in [46] with LPCVD-grown $\mathrm{Si}_{3} \mathrm{~N}_{4}$ waveguides. 


\subsection{Dependence on Waveguide Width}

In order to extend the supercontinuum further towards the mid-infrared side of the spectrum, we recall that the long-wavelength zero-dispersion wavelength in Fig. 3.1 shifts to larger values with increasing waveguide width. The dispersive waves result from a coherent energy transfer from the soliton in the anomalous dispersion regime to phase-matched frequencies in the normal dispersion regime, where the phase matching depends on the phase- and group velocity of the soliton as well as its peak power [1,52-54]. This suggests that the infrared side of the SC would shift to longer wavelengths as well. For a verification we carried out spectral measurements vs. increasing waveguide width. Fig. 3.4 shows the spectral power density recorded with seven different widths (from 0.7 to $1.3 \mu \mathrm{m}$ ) at the same, maximum available pump pulse energy of $6.8 \mathrm{~nJ}$ incident on the aspheric lens.

The dotted line indicates the change of the $-30 \mathrm{~dB}$ limit at the long-wavelength side of the spectrum. It can be clearly seen that the infrared end of the spectrum extends progressively towards mid-infrared wavelengths with increasing waveguide width. The observable wavelength shift is about $2.45 \mathrm{~nm}$ per $\mathrm{nm}$ waveguide width. A further progression and extension of the spectra to wavelengths beyond $2.6 \mu \mathrm{m}$ seems present for the three widest waveguides $(1.0$ to $1.3 \mu \mathrm{m})$ as well, but could not be recorded with the available spectroscopic equipment. Our theoretical model predicts the SC extending to wavelengths of 2.6, 2.7, 2.8 and $2.9 \mu \mathrm{m}$ for waveguide widths of 1.1, 1.2, 1.3 and $1.4 \mu \mathrm{m}$, respectively, while reproducing the experiment value for the smaller widths. The shift in location of the long-wavelength dispersive wave is reduced to about $1 \mathrm{~nm}$ per $\mathrm{nm}$ waveguide widening for widths larger than $1.1 \mu \mathrm{m}$.

The widest of all generated spectra, within the available detection range, was obtained with a $1-\mu \mathrm{m}$ wide waveguide and a pump pulse energy incident on the aspheric lens of $4.7 \mathrm{~nJ}(1.4 \mathrm{~nJ}$ waveguide internal). The spectrum is displayed in Fig. 3.5. From its short-wavelength $-30 \mathrm{~dB}$-edge at $526 \mathrm{~nm}$ it extends to at least $2.584 \mu \mathrm{m}$ (mid-infrared end of OSA detection range), which corresponds to a bandwidth of more than $454 \mathrm{THz}$ and spans at least 2.2 octaves.

\subsection{Summary and Conclusions}

In summary, we have demonstrated extremely wide bandwidth of supercontinuum generation in stoichiometric $\mathrm{Si}_{3} \mathrm{~N}_{4}$ waveguides, using a standard erbium-doped fiber pump laser that provided ultrashort pulses in the telecommunication wavelength range at $1560 \mathrm{~nm}$. In a $6 \mathrm{~mm}$ long waveguide with a core area of $1.0 \times 0.9 \mu \mathrm{m}^{2}$ and pumped by $120 \mathrm{fs}$ long pulses with a pulse energy of $4.7 \mathrm{~nJ}$ incident on the 


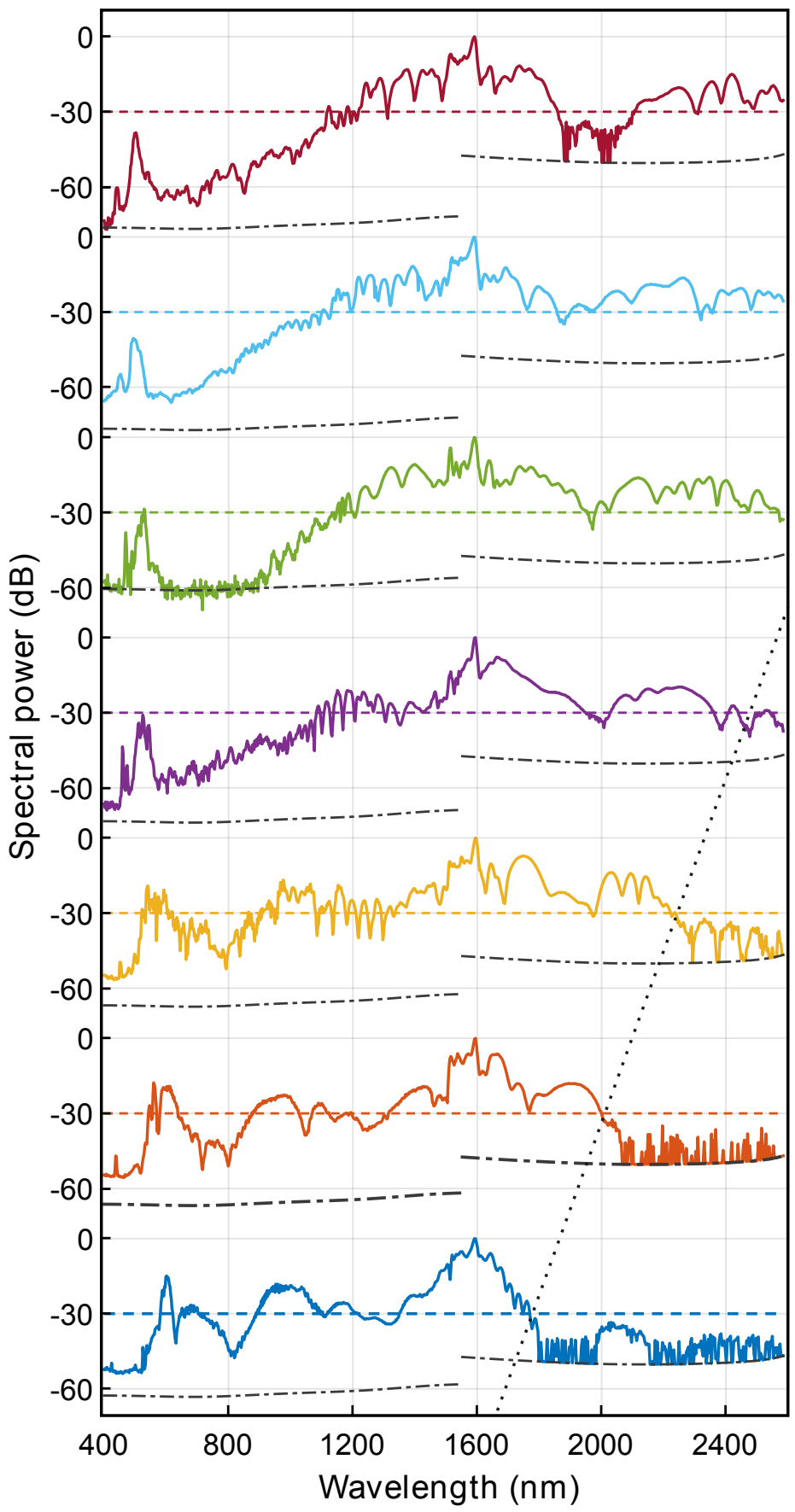

Figure 3.4: Measured spectral power density of supercontinuum generation using seven different waveguide widths, increasing from (blue trace) $0.7 \mu \mathrm{m}$ (blue trace) to $1.3 \mu \mathrm{m}$ (red trace) in steps of $0.1 \mu \mathrm{m}$, pumped with the same, maximum available pump pulse energy of $6.8 \mathrm{~nJ}$ incident on the aspheric lens. For clarity the peak values of all spectra are normalized to $0 \mathrm{~dB}$ and vertically offset by $80 \mathrm{~dB}$ with respect to each other. The horizontal dashed lines mark the respective $-30 \mathrm{~dB}$ levels. The dash-dotted curves show the average noise levels for the short-wavelength OSA, and a baseline after dark-count subtraction for the long-wavelength OSA. The straight dotted line shows that the $30 \mathrm{~dB}$ infrared-end of the spectra tunes towards the mid-infrared range with increasing width of the waveguides. 


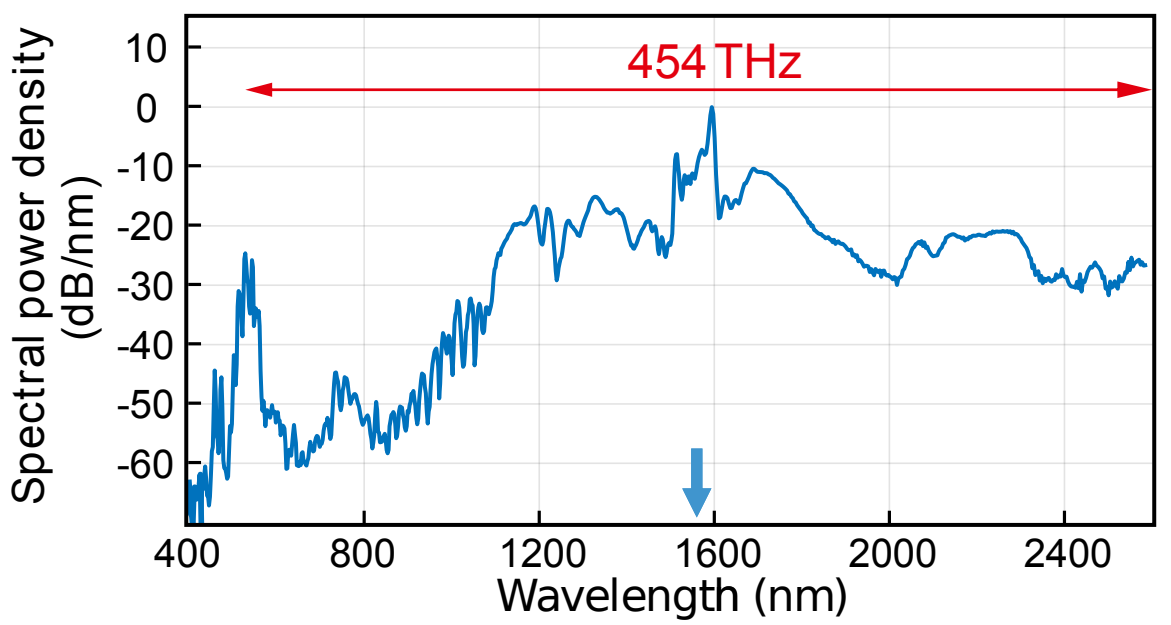

Figure 3.5: Supercontinuum spectrum generated in a $1 \mu \mathrm{m}$ wide and $0.9 \mu \mathrm{m}$ high waveguide using a pump pulse energy of $4.7 \mathrm{~nJ}$ incident to the aspheric lens (in-coupled pulse energy $1.4 \mathrm{~nJ}$ ). The pump wavelength of $1560 \mathrm{~nm}$ is indicated with an arrow. From the shortwavelength $-30 \mathrm{~dB}$-edge at $526 \mathrm{~nm}$ the spectrum extends to at least $2584 \mathrm{~nm}$ (mid-infrared end of OSA detection range). This corresponds to a spectral bandwidth of more than $454 \mathrm{THz}$ and spans more than 2.2 octaves.

aspheric lens, the supercontinuum radiation showed a spectral bandwidth of more than $454 \mathrm{THz}$, spanning at least 2.2 octaves, from the visible at $526 \mathrm{~nm}$ to the midinfrared (beyond $2584 \mathrm{~nm}$ ). The shapes of the generated spectra are in very good agreement with theoretical modeling via numerical integration of the generalized nonlinear Schrödinger equation (GNLSE). Based on this agreement a comparison of the required waveguide-internal pump pulse energy in the simulation and the measurements has been performed. The comparison revealed a factor of 3.6 lower pulse energy required in the simulations. A careful analysis suggests that the nonlinear refractive index of LPCVD-grown stoichiometric silicon nitride, $\mathrm{Si}_{3} \mathrm{~N}_{4}$, is smaller by a factor of about four than the previously reported value for PECVD-grown silicon nitride [46] and smaller by a factor of 1.8 than the previously reported value for LPCVD-grown $\mathrm{Si}_{3} \mathrm{~N}_{4}$ [45]. A further clarification should be attempted, such as with direct index modulation experiments using LPCVD-grown $\mathrm{Si}_{3} \mathrm{~N}_{4}$ waveguides.

Shifting the long-wavelength edge of the supercontinuum generation towards the mid-infrared with increasing waveguide width was achieved in agreement with engineering the waveguide dispersion. In the experiment a wavelength shifting of $2.45 \mathrm{~nm}$ per nm waveguide widening of the $-30 \mathrm{~dB}$ level at the infrared-side of the spectrum was observed, for waveguide widths between $0.7 \mu \mathrm{m}$ and $1.0 \mu \mathrm{m}$. We note that following our primary report on mid-infrared shifting [55] there was a subsequent observation [56] analogous to our results. Furthermore, the high index 
contrast provides tight guiding which concentrates the optical mode predominantly in the $\mathrm{Si}_{3} \mathrm{~N}_{4}$ core, where mid-infrared absorption is weaker [57]. The results imply that highly efficient supercontinuum generation appears feasible, if a low-loss input coupling can be obtained. We conclude this from the consistency between transmission loss measurements and detailed loss estimates, as well as from the good agreement between the shape of the measured and numerically calculated spectra. In these calculations only a low propagation loss and a negligible Raman conversion were assumed, thereby yielding a waveguide-internal conversion efficiency of typically $93 \%$. The measurements presented here, in agreement with mode matching calculations, show that the input coupling may be substantially increased by combining tighter focusing and tapered waveguides $[27,58]$. With these properties and options, stoichiometric silicon nitride waveguides pumped with mode-locked telecommunication laser sources provide an interesting platform for various applications, including multiple-octave spanning frequency combs with self-referencing for frequency metrology [59], or mid-infrared dual comb spectroscopy [60].

\section{References}

1. Dudley, J. M., Genty, G. \& Coen, S. Supercontinuum generation in photonic crystal fiber. Rev. Mod. Phys. 78, 1135-1184 (2006).

2. Jones, D. J., Diddams, S. A., Ranka, J. K., Stentz, A., Windeler, R. S., Hall, J. L. \& Cundiff, S. T. Carrier-Envelope Phase Control of Femtosecond Mode-Locked Lasers and Direct Optical Frequency Synthesis. Science 288, 635-639 (2000).

3. Ruehl, A. et al. Ultrabroadband coherent supercontinuum frequency comb. Phys. Rev. A 84, 011806 (2011).

4. Kano, H. \& Hamaguchi, H.-o. Characterization of a supercontinuum generated from a photonic crystal fiber and its application to coherent Raman spectroscopy. Opt. Lett. 28, 2360-2362 (2003).

5. Humbert, G. et al. Supercontinuum generation system for optical coherence tomography based on tapered photonic crystal fibre. Opt. Express 14, 1596-1603 (2006).

6. Woodward, J. T., Smith, A. W., Jenkins, C. A., Lin, C., Brown, S. W. \& Lykke, K. R. Supercontinuum sources for metrology. Metrologia 46, S277 (2009).

7. Ycas, G., Osterman, S. \& Diddams, S. A. Generation of a $6602100 \mathrm{~nm}$ laser frequency comb based on an erbium fiber laser. Opt. Express 37, 2199 (2012).

8. Brasch, V., Herr, T., Geiselmann, M., Lihachev, G., Pfeiffer, M. H. P., Gorodetsky, M. L. \& Kippenberg, T. J. Photonic chip based optical frequency comb using soliton induced Cherenkov radiation. Science 351, 357-360 (2016).

9. Pfeifle, J. et al. Coherent terabit communications with microresonator Kerr frequency combs. Nat. Photon. 8, 375-380 (2014).

10. Torres-Company, V. \& Weiner, A. M. Optical frequency comb technology for ultrabroadband radio-frequency photonics. Laser Photon. Rev. 8, 368-393 (2014). 
11. Nguyen, T. G., Shoeiby, M., Chu, S. T., Little, B. E., Morandotti, R., Mitchell, A. \& Moss, D. J. Integrated frequency comb source based Hilbert transformer for wideband microwave photonic phase analysis. Opt. Express 23, 22087 (2015).

12. K L Corwin, N. R. N. Broadband noise on supercontinuum generated in microstructure fiber. OSA Trends in Optics and Photonics (TOPS) Nonlinear Optics 79, 409-411 (2002).

13. Kuyken, B. et al. Nonlinear optical interactions in silicon waveguides. Nanophoton. 6, 377-392 (2017).

14. Ciret, C. \& Gorza, S.-P. Generation of ultra broadband coherent supercontinuum in tapered and dispersion managed silicon nanophotonic waveguides. J. Opt. Soc. A. B 34, 1156-1163 (2017).

15. Hu, H., Li, W. \& Dutta, N. K. Dispersion-engineered tapered planar waveguide for coherent supercontinuum generation. Opt. Commun. 324, 252-257 (2014).

16. Oh, D. Y., Sell, D., Lee, H., Yang, K. Y., Diddams, S. A. \& Vahala, K. J. Supercontinuum generation in an on-chip silica waveguide. Opt. Lett. 39, 1046 (2014).

17. Duchesne, D., Peccianti, M., Lamont, M. R. E., Ferrera, M., Razzari, L., Légaré, F., Morandotti, R., Chu, S., Little, B. E. \& Moss, D. J. Supercontinuum generation in a high index doped silica glass spiral waveguide. Opt. Express 18, 923 (2010).

18. Halir, R., Okawachi, Y., Levy, J. S., Foster, M. A., Lipson, M. \& Gaeta, A. L. Ultrabroadband supercontinuum generation in a CMOS-compatible platform. Opt. Lett. 37, 1685 (2012).

19. Chavez Boggio, J. M., Bodenmüller, D., Fremberg, T., Haynes, R., Roth, M. M., Eisermann, R., Lisker, M., Zimmermann, L. \& Böhm, M. Dispersion engineered silicon nitride waveguides by geometrical and refractive-index optimization. J. Opt. Soc. Am. B 31, 2846 (2014).

20. Lamont, M. R., Luther-Davies, B., Choi, D.-Y., Madden, S. \& Eggleton, B. J. Supercontinuum generation in dispersion engineered highly nonlinear $(\gamma=10 / \mathrm{W} / \mathrm{m}) \mathrm{As}_{2} \mathrm{~S}_{3}$ chalcogenide planar waveguide. Opt. Express 16, 14938-14944 (2008).

21. Yu, Y., Gai, X., Ma, P., Choi, D.-Y., Yang, Z., Wang, R., Debbarma, S., Madden, S. J. \& Luther-Davies, B. A broadband, quasi-continuous, mid-infrared supercontinuum generated in a chalcogenide glass waveguide. Laser Photon. Rev. 8, 792-798 (2014).

22. Leo, F., Gorza, S.-P., Safioui, J., Kockaert, P., Coen, S., Dave, U., Kuyken, B. \& Roelkens, G. Dispersive wave emission and supercontinuum generation in a silicon wire waveguide pumped around the $1550 \mathrm{~nm}$ telecommunication wavelength. Opt. Lett. 39, 3623 (2014).

23. Lau, R. K. W., Lamont, M. R. E., Griffith, A. G., Okawachi, Y., Lipson, M. \& Gaeta, A. L. Octave-spanning mid-infrared supercontinuum generation in silicon nanowaveguides. Opt. Lett. 39, 4518 (2014).

24. Kuyken, B. et al. An octave-spanning mid-infrared frequency comb generated in a silicon nanophotonic wire waveguide. Nat. Commun. 6, 6310 (2015).

25. Liu, X., Pu, M., Zhou, B., Krückel, C. J., Fülöp, A., Torres-Company, V. \& Bache, M. Octave-spanning supercontinuum generation in a silicon-rich nitride waveguide. Opt. Lett. 41, 2719-2722 (2016).

26. Morichetti, F., Melloni, A., Martinelli, M., Heideman, R. G., Leinse, A., Geuzebroek, D. H. \& Borreman, A. Box-Shaped Dielectric Waveguides: A New Concept in Integrated Optics? J. Lightwave Technol., JLT 25, 2579-2589 (1, 2007). 
27. Wörhoff, K., Heideman, R. G., Leinse, A. \& Hoekman, M. TriPleX: a versatile dielectric photonic platform. Adv. Opt. Technol. 4, 189-207 (2015).

28. Oldenbeuving, R. M., Klein, E. J., Offerhaus, H. L., Lee, C. J., Song, H. \& Boller, K.-J. $25 \mathrm{kHz}$ narrow spectral bandwidth of a wavelength tunable diode laser with a short waveguide-based external cavity. Laser Phys. Lett. 10, 015804 (2013).

29. Fan, Y., Oldenbeuving, R. M., Klein Edwin J.and Lee, C. J., Song, H., Khan, M. R. H., Offerhaus, H. L., van der Slot, P. J. M. \& Boller, K.-J. A hybrid semiconductor-glass waveguide laser. Proc.SPIE 9135 (2014).

30. Gondarenko, A., Levy, J. S. \& Lipson, M. High confinement micron-scale silicon nitride high Q ring resonator. Opt. Express 17, 11366-11370 (2009).

31. Bauters, J. F., Heck, M. J. R., John, D., Dai, D., Tien, M.-C., Barton, J. S., Leinse, A., Heideman, R. G., Blumenthal, D. J. \& Bowers, J. E. Ultra-low-loss high-aspect-ratio $\mathrm{Si}_{3} \mathrm{~N}_{4}$ waveguides. Opt. Express 19, 3163-3174 (2011).

32. Xiong, C. et al. Compact and reconfigurable silicon nitride time-bin entanglement circuit. Optica 2, 724 (2015).

33. Zhuang, L., Leinse, A., Heideman, R. G., van Dijk, P. W. L. \& Roeloffzen, C. G. H. Integrated microwave photonic signal processors in TriPleX TM waveguide in J. Lightwave Technol. (IEEE, 2013), 1-1.

34. Roeloffzen, C. G. H., Zhuang, L., Taddei, C., Leinse, A., Heideman, R. G., van Dijk, P. W. L., Oldenbeuving, R. M., Marpaung, D. A. I., Burla, M. \& Boller, K.-J. Silicon nitride microwave photonic circuits. Opt. Express 21, 22937 (2013).

35. Zhuang, L., Roeloffzen, C. G. H., Hoekman, M., Boller, K.-J. \& Lowery, A. J. Programmable photonic signal processor chip for radiofrequency applications. Optica 2, 854-859 (2015).

36. Klenner, A., Mayer, A. S., Johnson, A. R., Luke, K., Lamont, M. R. E., Okawachi, Y., Lipson, M., Gaeta, A. L. \& Keller, U. Gigahertz frequency comb offset stabilization based on supercontinuum generation in silicon nitride waveguides. Opt. Express 24, 11043 (2016).

37. Epping, J. P. et al. On-chip visible-to-infrared supercontinuum generation with more than $495 \mathrm{THz}$ spectral bandwidth. Opt. Express 23, 19596-19604 (2015).

38. Johnson, A. R. et al. Octave-spanning coherent supercontinuum generation in a silicon nitride waveguide. Opt. Lett. 40, 5117 (2015).

39. Mayer, A. S., Klenner, A., Johnson, A. R., Luke, K., Lamont, M. R. E., Okawachi, Y., Lipson, M., Gaeta, A. L. \& Keller, U. Frequency comb offset detection using supercontinuum generation in silicon nitride waveguides. Opt. Express 23, 15440 (2015).

40. Agrawal, G. P. Nonlinear Fiber Optics (Academic Press, 2007).

41. Epping, J. P., Hoekman, M., Mateman, R., Leinse, A., Heideman, R. G., van Rees, A., van der Slot, P. J. M., Lee, C. J. \& Boller, K.-J. High confinement, high yield $\mathrm{Si}_{3} \mathrm{~N}_{4}$ waveguides for nonlinear optical applications. Opt. Express 23, 642-648 (2015).

42. Hardin, R. H. \& Tappert, F. D. Applications of the split-step Fourier method to the numerical solution of nonlinear and variable coefficient wave equations in. SIAM-SIREAD. 15 (1973), 423.

43. Taha, T. R. \& Ablowitz, M. I. Analytical and numerical aspects of certain nonlinear evolution equations. II. Numerical, nonlinear Schrödinger equation. J. Comp. Phys. 55, 203-230 (1984). 
44. Kues, M., Brauckmann, N., Walbaum, T., GroSS, P. \& Fallnich, C. Nonlinear dynamics of femtosecond supercontinuum generation with feedback. Opt. Express 17, 1582715841 (2009).

45. Levy, J. Integrated Nonlinear Optics In Silicon Nitride Waveguides And Resonators PhD thesis (2011).

46. Ikeda, K., Saperstein, R. E., Alic, N. \& Fainman, Y. Thermal and Kerr nonlinear properties of plasma-deposited silicon nitride/ silicon dioxide waveguides. Opt. Express 16, 12987-12994 (2008).

47. Liu, X.-J., Zhang, J.-J., Sun, X.-W., Pan, Y.-B., Huang, L.-P. \& Jin, C.-Y. Growth and properties of silicon nitride films prepared by low pressure chemical vapor deposition using trichlorosilane and ammonia. Thin Solid Films 460, 72-77 (2004).

48. Krückel, C. J., Fülöp, A., Klintberg, T., Bengtsson, J., Andrekson, P. A. \& Torres-Company, $\mathrm{V}$. Linear and nonlinear characterization of low-stress high-confinement silicon-rich nitride waveguides. Opt. Express 23, 25827 (2015).

49. Zhao, H. et al. Visible-to-near-infrared octave spanning supercontinuum generation in a silicon nitride waveguide. Opt. Express 40, 2177-2180 (2015).

50. Karasawa, N., Nakamura, S., Nakagawa, N., Shibata, M., Morita, R., Shigekawa, H. \& Yamashita, M. Comparison between theory and experiment of nonlinear propagation for a-few-cycle and ultrabroadband optical pulses in a fused-silica fiber. IEEE J. Quant. Electron. 37, 398-404 (2001).

51. Epping, J. P. Dispersion engineering silicon nitride for broadband nonlinear frequency conversion $\mathrm{PhD}$ thesis (2015).

52. Akhmediev, N. \& Karlsson, M. Cherenkov radiation emitted by solitons in optical fibers. Phys. Rev. A 51, 2602-2607 (1995).

53. Tartara, L., Cristiani, I. \& Degiorgio, V. Blue light and infrared continuum generation by soliton fission in a microstructured fiber. Appl. Phys. B 77, 307-311 (2003).

54. Cristiani, I., Tediosi, R., Tartara, L. \& Degiorgio, V. Dispersive wave generation by solitons in microstructured optical fibers. Opt. Express 12, 124-135 (2004).

55. Schepers, F. et al. Ultra-broadband Supercontinuum Generation at Telecommunication Wavelengths in Dispersion Engineered Stoichiometric $\mathrm{Si}_{3} \mathrm{~N}_{4}$ Waveguides in Conference on Lasers and Electro-Optics (2016) CLEO: Applications and Technology (Optical Society of America, 2016), AM3J.5.

56. Grassani, D., Billat, A., Pfeiffer, M. H. P., Guo, H., North, T., Kippenberg, T. J. \& Bres, C.-S. Mid-infrared supercontinuum generation in a SiN waveguide pumped at 1.55 micron in FTu5D.3 Frontiers in Optics (Optical Society of America, 2016).

57. Liao, B.-H. \& Hsiao, C.-N. Improving optical properties of silicon nitride films to be applied in the middle infrared optics by a combined high-power impulse/unbalanced magnetron sputtering deposition technique. Appl. Opt. 53, A377 (2014).

58. Hosseinnia, A. H., Atabaki, A. H., Eftekhar, A. A. \& Adibi, A. High-quality silicon on silicon nitride integrated optical platform with an octave-spanning adiabatic interlayer coupler. Opt. Express 23, 30297 (2015).

59. Chembo, Y. K. Kerr optical frequency combs: theory, applications and perspectives. Nanophoton. 5, 214-230 (2016).

60. Schliesser, A., Picqué, N. \& Hänsch, T. W. Mid-infrared frequency combs. Nat. Photon. 6, 440-449 (2012). 



\section{Chapter 4}

\section{Refractive index modulation via surface acoustic waves}

We theoretically investigate the use of Rayleigh surface acoustic waves (SAWs) for refractive index modulation in optical waveguides consisting of amorphous dielectrics. Considering low-loss $\mathrm{Si}_{3} \mathrm{~N}_{4}$ waveguides with a standard core cross section of $4.4 \times 0.03 \mu \mathrm{m}^{2}$ size, buried $8 \mu \mathrm{m}$ deep in a $\mathrm{SiO}_{2}$ cladding we compare surface acoustic wave generation in various different geometries via a piezo-active, lead-zirconatetitanate film placed on the surface and driven via an interdigitized transducer (IDT). Using numerical solutions of the acoustic and optical wave equations, we determine the strain distribution of SAW modes under resonant excitation, and the electric field distribution of the fundamental optical mode near the waveguide core. From the overlap of the acoustic strain field with the optical mode field we calculate and maximize the attainable amplitude of index modulation in the waveguide. For the example of a near-infrared wavelength of $840 \mathrm{~nm}$, a maximum relative refractive index modulation of $1.4 \times 10^{-3}$ was obtained for an IDT periodicity of $30 \mu \mathrm{m}$, a film thickness of $4 \mu \mathrm{m}$, and the IDT voltage and modulation frequency were $10 \mathrm{~V}$ and $90 \mathrm{MHz}$, respectively. The relative index change is $1.4 \times 10^{-3}$, which is about 300-times higher than in previous work based on non-resonant proximity piezo-actuation, and the modulation frequency is about 200 -times higher. Exploiting the relative index change of $1.4 \times 10^{-3}$ in a Mach-Zehnder modulator should allow full-contrast modulation in devices as short as $103 \mu \mathrm{m}$. 


\subsection{Introduction}

Integrated optical waveguides fabricated from stoichiometric silicon nitride $\left(\mathrm{Si}_{3} \mathrm{~N}_{4}\right)$ using low pressure chemical vapor deposition (LPCVD) can offer ultra-low loss, are transparent from visible to near-infrared wavelengths $[1,2]$ and, therefore, are of high interest for numerous applications. These include communications [3], sources for nonlinear microscopy [4], optical coherence tomography [5], biosensors [6], microwave photonics [7, 8], ultra-narrow bandwidth hybrid lasers [9] and supercontinuum generation $[4,10]$. Due to a high index contrast with regard to the $\mathrm{SiO}_{2}$ cladding, these waveguides enable dense, complex and reconfigurable integrated photonic circuits [11-13].

Typically, light modulation in silicon nitride waveguides relies on the thermo-optic effect and is based on a thermally induced phase shift between the two arms of a Mach-Zehnder interferometer [14]. State-of-the-art thermo-optic modulators provide up to $1 \mathrm{kHz}$ modulation speed, while the dissipation of heating power is often undesired, because it can be as large as $500 \mathrm{~mW}$ per modulator [7]. Applications that rely on a high density of modulators, e.g., as in reconfigurable photonic circuits [12], would greatly benefit from modulation techniques with lower dissipation, while applications needing fast modulation of the light would greatly benefit from techniques with higher switching or modulation speeds. A technique, which may provide both benefits simultaneously, may be based on the strain-optic effect $[15,16]$ where stress induced in the waveguide results in a change of the effective refractive index. The strain-optic effect has been studied in various integrated photonic systems $[17,18]$. Specifically in the waveguide platform investigated here (LPCVD $\mathrm{Si}_{3} \mathrm{~N}_{4} / \mathrm{SiO}_{2}$ ) there is so far only a single implementation. In [18], Hosseini et al. showed an approach with a $2 \mu \mathrm{m}$-thick layer of crystalline lead zirconate titanate (PZT) deposited on top of a silicon nitride Mach-Zehnder interferometer (MZI) with the core of the waveguides positioned $8 \mu \mathrm{m}$ below the PZT layer. Via an electrode placed on top of the PZT layer above one of the interferometer arms of the MZI, the stress within that arm could be locally controlled via the electrode voltage. The power consumption was reduced significantly, by six order of magnitude. Also the modulation frequency could be raised, up to $600 \mathrm{kHz}$ (at $-3 \mathrm{db}$ bandwidth). However, further increasing the modulation frequency was not possible. In that approach this would require a smaller capacitance resulting in a reduced electrode area, but this would reduce the induced stress and thereby the induced index change. The maximum index modulation remained rather small, at around 5 $\times 10^{-6}$ with an optimum geometry. This reduces the overall induced phase shift as required for decreasing the arm length of the interferometer.

Much higher modulation frequencies are possible with a more sophisticated electrode structure, using so-called interdigital transducers (IDTs) that resonantly excite 
surface acoustic waves (SAWs). For instance, optical modulation at a frequency of $520 \mathrm{MHz}$ was demonstrated for a compact MZI consisting of conventional ridge waveguides of GaAs with an active region of only $15 \mu \mathrm{m}$ [19], while acousto-optic modulation of photonic resonators on thin polycrystalline aluminum nitride films has been demonstrated for modulation frequencies reaching well into the microwave range [20,21]. In $\mathrm{Si}_{3} \mathrm{~N}_{4}$ stress-induced optical modulation at frequencies above the $\mathrm{MHz}$ are not yet available.

Here, we theoretically investigate exploiting SAW-induced effective refractive index changes for realizing faster and smaller modulators with $\mathrm{Si}_{3} \mathrm{~N}_{4}$ waveguides. Using numerical methods we calculate the index modulation experienced by the fundamental optical mode propagating through a $\mathrm{Si}_{3} \mathrm{~N}_{4}$ core buried in a $\mathrm{SiO}_{2}$ cladding. The special interest in this particular geometry is that no deterioration of the ultralow optical propagation loss is expected. The reason for that is that the cladding is assumed to be thick enough to make the optical field negligible at the location of the thin PZT film that is on top of the cladding. At the same time, the penetration depth of SAWs is large enough, on the order of the acoustic wavelength, $\lambda_{\mathrm{R}}$, in the material $[22,23]$, which allows for a good overlap of the SAW with the optical wave even for high modulation frequencies in the $100 \mathrm{MHz}$ range. The SAW is considered to be launched using an IDT. Compared to the unstructured electrode arrangement used by Hosseini et al. [18], we show below that the fine structuring of the IDT allows typically 200-times higher modulation frequencies while resonant excitation yields a 300-fold increase in index modulation. Another advantage of employing SAWs is that tensile strain can be applied in one interferometer arm, simultaneously applying compressive strain in the other arm, which effectively reduces the length of the arms by a factor of two to obtain full light modulation [19].

In the following we consider acousto-optic modulation using a MZI, in a setting where the acoustic wave propagates perpendicular to the optical waveguide axis of the two arms of the MZI as shown in Fig. 4.1. We briefly discuss the relation between strain and the refractive index and the surface acoustic wave of interest. We then present the geometry studied and how the simulations are performed. We investigate how the induced strain and, consequently, the effective refractive index, depends on the thickness of the PZT layer and the period of the IDT used to generated the SAW. Finally, we use the maximum change in effective refractive index to determine the required length of the MZI to obtain full modulation of the optical wave. The maximum relative change of the effective waveguide index in our arrangement was found to be $0.14 \%$, at a frequency of $90 \mathrm{MHz}$, which yields full modulation with a relatively short arm length of $103 \mu \mathrm{m}$, i.e., about 80-times shorter than the proximity piezo methods described above. 


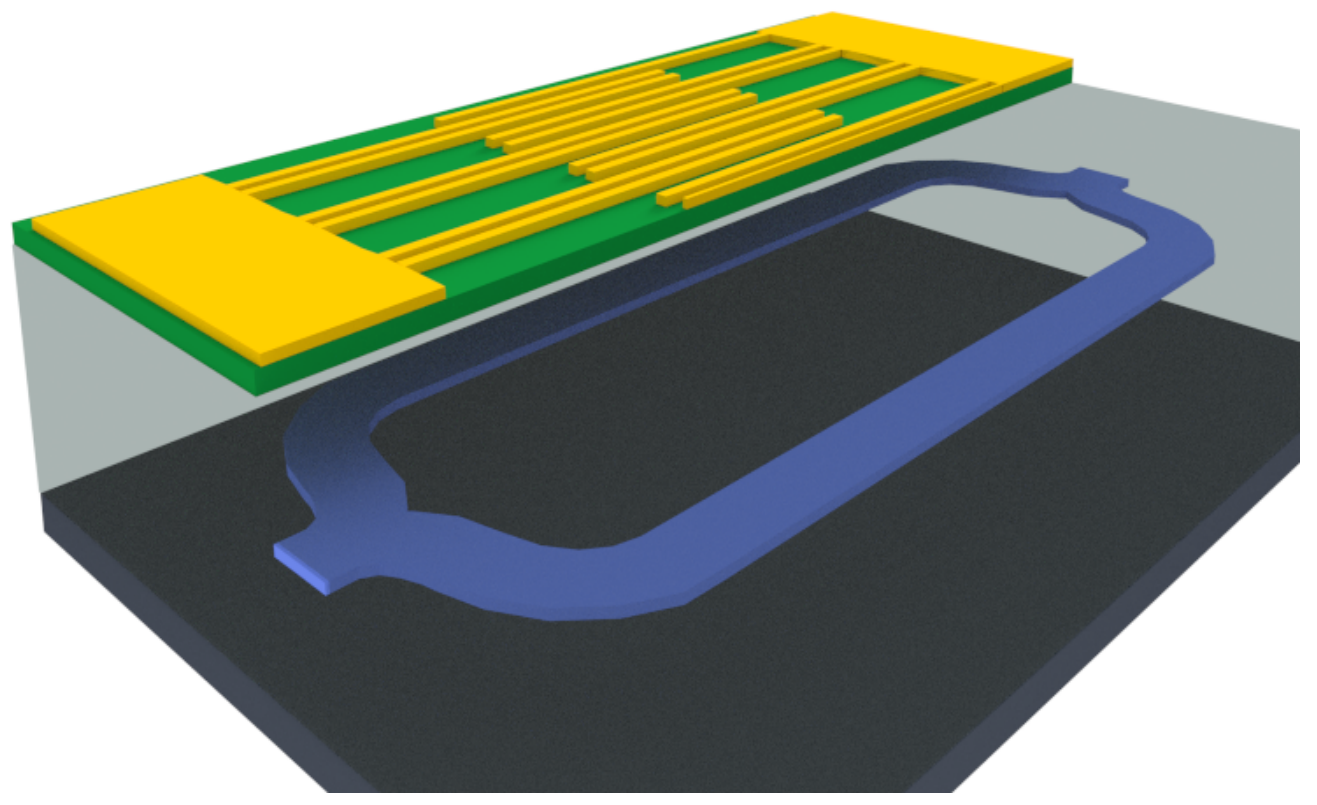

Figure 4.1: Artistic impression of a waveguide (in blue) based MachZehnder interferometer. Half of the electrode structure and piezo layer is not shown to enable a full view on the interferometer (dark blue waveguides). The interferometer waveguides are buried in $\mathrm{SiO}_{2}$ (light gray) deposited on a silicon substrate (dark gray).

\subsection{Acousto-optic refractive index modulation}

The response of a material to an applied electric field, an acoustic wave, or a combination of the two, strongly depends on the type of material. Here, we are interested on one hand in generating a strong acoustic wave using the piezoelectric effect $[24,25]$. On the other hand, we intend to use strain in amorphous waveguide core $\left(\mathrm{Si}_{3} \mathrm{~N}_{4}\right)$ and cladding $\left(\mathrm{SiO}_{2}\right)$ materials (which do not possess a piezoelectric effect) to cause strain-induced changes in refractive index [24, 25]. The strain can be compressive or tensile, leading to an increase or decrease in local refractive index, respectively. In a microscopic picture, strain changes both the number of microscopic dipoles per unit volume and the microscopic potential. This volume change determines the induced dipole driven by the applied optical field and thus changes the optical susceptibility tensor, $\chi$, of the material. In the most general case of an anisotropic material, the relation between the change in the inverse of the dielectric tensor and the applied strain is given by $[16,26]$,

$$
\left[\Delta\left(\varepsilon^{-1}\right)\right]_{i j}=(\mathbf{p S})_{i j}=\sum_{k l} \mathbf{p}_{i j k l} \mathbf{S}_{k l}
$$


where $\varepsilon=\varepsilon_{0}(\mathbf{1}+\chi)$ is the dielectric tensor, $\varepsilon_{0}$ is the vacuum permittivity, $\mathbf{S}$ is the strain tensor and $\mathbf{p}$ is the dimensionless strain-optic tensor. The indices, $i, j, k$ and $l$ designate the three Cartesian coordinates. For isotropic materials such as the amorphous materials investigated here, and assuming small changes in the inverse dielectric tensor, eq. 4.1 can be simplified. In this case, the related change in refractive index is given by $[15,27]$

$$
\Delta n_{x}=-\frac{1}{2} n_{0}^{3}\left(p_{11} S_{x}+p_{12} S_{y}\right) .
$$

Here, $\Delta n_{x}$ is the change in refractive index for linear polarized light along the $x$ direction, $n_{0}$ is the refractive index of the material in absence of any strain, $S_{i}$ is the strain applied in the $i$-direction $(i=x, y)$ and the contracted indices notation is used [16]. The change in refractive index for the other polarization direction is obtained via exchanging the strain-tensor components,

$$
\Delta n_{y}=-\frac{1}{2} n_{0}^{3}\left(p_{12} S_{x}+p_{11} S_{y}\right)
$$

In this work, strain in the region of the optical mode is induced by an acoustic wave. As the optical mode is confined to an area just a few micrometer below the surface of the cladding, for obtaining a strong interaction, the surface acoustic wave (SAW) is the most appropriate acoustic wave to consider for a strong interaction. The reason is that a SAW travels along the surface of an elastic material with most of its energy and strain confined within a small region, with a thickness of the order of the acoustic wavelength, below the surface and thus can provide good overlap between the strain induced by the SAW and the optical mode. Of various different surface waves possible, we focus on Rayleigh surface acoustic waves, which are characterized by a correlated transverse and longitudinal motion at the surface. This results in volume elements traversing an elliptical path when the wave passes [28]. The motivation to investigate Rayleigh waves is that the considered SAW ${ }^{1}$ has low dispersion, as long as the elastic modulus near the surface does not change [28], making them suitable for the modulation of broadband signals [29].

If the SAW is launched perpendicular to the two arms of a MZI as depicted in Fig 4.2, and the two arms of length $L$ are separated by half an acoustic wavelength, one arm experiences compressive strain and the other tensile strain. The phase shift of light leaving either interferometer arm with respect to the light entering the interferometer is given by

$$
\Delta \varphi=2 \pi \Delta n \frac{L}{\lambda}
$$

where $\Delta n$ is the change in effective refractive index, $n_{\text {eff }}$, of the optical mode, which is opposite in sign for the two arms, $n_{\mathrm{eff}}=\frac{\beta \lambda}{2 \pi}, \beta$ is the propagation constant for the

\footnotetext{
${ }^{1}$ SAW refers to a Rayleigh surface acoustic wave in the remainder of this chapter.
} 
fundamental mode, and $\lambda$ is the vacuum wavelength. When the light is combined at the output of the MZI, the total phase difference is

$$
\Delta \varphi_{t}=4 \pi|\Delta n| \frac{L}{\lambda} .
$$

A total phase difference of $\Delta \varphi_{t}=\pi$ is required for full light modulation. Therefore, when the length $L$ is equal to

$$
L=\frac{\lambda}{4|\Delta n|},
$$

light is fully modulated with a modulation frequency equal to the frequency of the SAW. The latter equation shows that a weak index modulation (small $\Delta n$ ) would require long arm lengths or interaction lengths, which is undesired for compact, integrated waveguide circuits with a high density of components.

For providing full modulation also with short interaction lengths, a SAW is ideally created in a material having a large piezoelectric coefficient. For our calculations we consider lead zirconate titanate (PZT), because PZT is known as high performance piezoelectric material and commonly used in actuators and sensors [30]. Another advantage is that thin PZT layers have already been successfully deposited on $\mathrm{Si}_{3} \mathrm{~N}_{4}$ waveguides in a $\mathrm{SiO}_{2}$ cladding [18] by using a Ti/Pt bilayer as seed for epitaxial growth of the PZT [18] on top of the amorphous $\mathrm{SiO}_{2}$ cladding material. The PZT layer may be grown using pulsed laser deposition [18] or alternative techniques like liquid-phase growth [31]. The different seeding techniques allow configurations with and without a conducting layer between the amorphous cladding and crystalline PZT. The generation and comparison of SAWs in various such geometries is presented in the next section.

\subsection{Geometry and simulation domain}

The geometries considered here are shown schematically in Fig. 4.2(a) and as crosssections in (b-f). The optical design comprises a typical low-loss optical waveguide having a $\mathrm{Si}_{3} \mathrm{~N}_{4}$ core of height $30 \mathrm{~nm}$ and width $4.4 \mu \mathrm{m}$ embedded symmetrically in a $16 \mu \mathrm{m}$ thick $\mathrm{SiO}_{2}$ cladding on top of a Si wafer substrate, i.e., at $8 \mu \mathrm{m}$ distance from the $\mathrm{Si}$ and $\mathrm{SiO}_{2}$ interfaces. The strain-optic tensor is not known for $\mathrm{Si}_{3} \mathrm{~N}_{4}$. However, due to the small core thickness the optical mode is mostly outside the core and a modulation of the effective refractive index is dominantly caused by the strain-optic effect in the $\mathrm{SiO}_{2}$ cladding. The thickness of the cladding, $8 \mu \mathrm{m}$, is taken big enough that the optical mode does not extend to the end of the cladding, in order to render optical losses due to surface layers or the substrate negligible. The top of the $\mathrm{SiO}_{2}$ cladding contains a thin conductive or dielectric seeding layer that 
allows the growth of c-oriented PZT. The thickness, $d$, of the PZT layer was varied to determine the optimum thickness for excitation of the SAW. A split-finger IDT configuration is used to excite the SAW without first order Bragg reflections [29]. In order to maximize the optical modulation amplitude, we investigate the effect of the location of the conductive (i.e. gold) electrodes of the IDT and a seeding layer in four different configurations.

The first consists of the IDT electrodes at the PZT-air interface and a conductive seeding layer at the $\mathrm{SiO}_{2}$-PZT interface (ETC, Fig. 4.2(c)). The second, consists of the IDT electrode on top of the PZT layer with a dielectric seed layer (shown in blue) (ETD, Fig. 4.2(d)). The third and fourth configurations have the IDT electrode at the $\mathrm{SiO}_{2}$-PZT interface and have a conductive layer (EBC, Fig. 4.2(e)) or simply a bare PZT-air interface (EBD, Fig. 4.2(f)). Configurations EBC and EBD have both a thin seed dielectric nanosheet deposited on top of the IDT electrode to allow crystalline growth of the PZT layer. Because the seed dielectric layer is only a few nm thick and is considered to have perfect adhesion to $\mathrm{SiO}_{2}$ it would not notably affect the acoustic wave, and is not included in the model.

The IDT generates a SAW and the associated strain induces a change in refractive index in both the cladding and the core. However, the optical modulation amplitude is expected to depend on the strain distribution of the excited SAW and its overlap with the optical mode. In order to determine the degree of overlap, the strain distribution is calculated by finding the fundamental SAW eigenmode for the four configurations using a finite-element eigenmode solver [32]. For these calculations, we use the two-dimensional unit cell shown in Fig. 4.2(b), which consists of one period of the split finger IDT electrode and the layers below (and layers above in case of configurations $\mathrm{EBC}$ and $\mathrm{EBD}$ ). To maximize the strain at the location of the optical mode, the waveguide core is positioned in the horizontal direction, $x$, in Fig. 4.2, to lie symmetrically underneath the gap of two IDT electrodes. Platinum is selected for the conductive seed layer material (gray) and gold (yellow) for the conductive layer on top of the PZT. Both layers as well as the gold electrodes of the IDT are taken as $100 \mathrm{~nm}$ thick.

The acoustic boundary conditions applied to the unit cell are a free displacement condition at the air interface, a zero displacement condition at the bottom of Si substrate and a periodic boundary condition at the two remaining boundaries. In order to ensure that the SAW has negligible amplitude near the bottom of the substrate and the zero displacement boundary condition does not affect the solution, the height of the Si substrate was found not to affect the solutions for heights larger than $5 \Lambda$, where $\Lambda$ is the period of the IDT electrode.

For the calculation of the optical field distribution, the optical boundary conditions were taken as zero-field conditions at all outer boundaries. This is well-justified because with the chosen index and size parameters the optical field is confined closely 


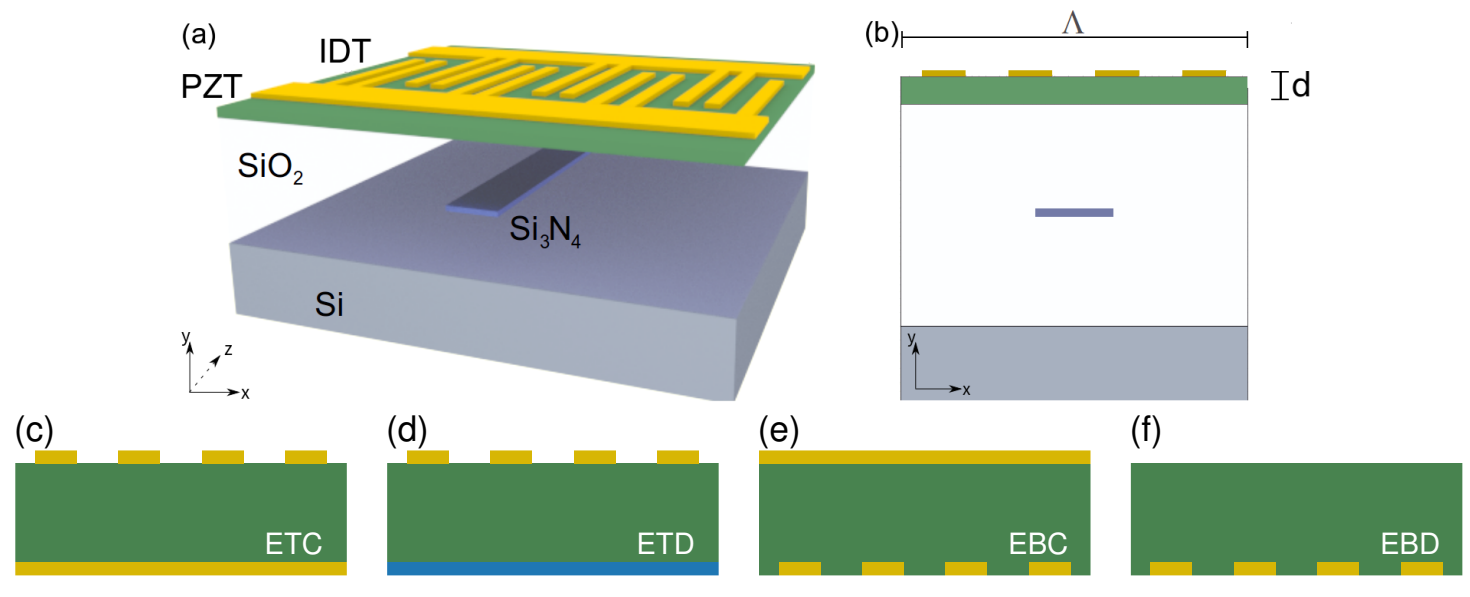

Figure 4.2: (a) Schematic view of the geometry comprising a $\mathrm{Si}_{3} \mathrm{~N}_{4}$ core ( $4.4 \times 0.03 \mu \mathrm{m}$, dark blue) centered in a $16-\mu \mathrm{m}$ thick $\mathrm{SiO}_{2}$ cladding (light gray) on a Si substrate (dark gray). A crystalline PZT layer on top of the cladding (green) in combination with an IDT (yellow) is used for exciting surface acoustic waves (SAWs). (b) The cross-section shows the corresponding two-dimensional unit cell across a single period $\Lambda$ of the IDT and the layers below it. The thickness of the Si layer included in the unit cell is proportional to $\Lambda$ to ensure a negligible SAW amplitude at the lower boundary of the unit cell. The layer between the PZT and the $\mathrm{SiO}_{2}$ layers is either conductive (yellow) or dielectric (light blue) and functions as seed layer for the crystalline growth of the PZT layer on top of the amorphous $\mathrm{SiO}_{2}$ or IDT electrode. (c)-(f) Illustrate the various combinations of IDT locations with and without opposite conductive layer, following the same color coding. (c) ETC, (d) ETD,(e) $\mathrm{EBC},(\mathrm{f}) \mathrm{EBD}$. Features are not to scale.

around the core as compared to the thickness of the cladding.

To calculate the resonant acoustic frequencies for each of the configurations as a function of both the thickness, $d$, of the PZT layer and period, $\Lambda$, of the IDT electrode, we used an eigenmode solver. Note that a resonant frequency corresponds to the modulation frequency of the light in a properly configured Mach-Zehnder interferometer. To determine the effective refractive index at the location of the optical mode, it is required that the applied voltage is chosen to oscillate at the resonant frequency. The electro-mechanical coupling coefficient was obtained by performing a frequency-domain simulation using the same unit cell as shown in Fig. 4.2(b) and calculating the strain distribution when a sinusoidal voltage with a given amplitude (we chose $10 \mathrm{~V}$ ) and a frequency equal to the resonant acoustic frequency is applied to the IDT electrode. Also for this study, we investigated the dependence of the induced strain on $d$ and $\Lambda$. The various isotropic material properties used in the 
Table 4.1: Material constants used in calculating the SAW properties. Parameters not included in the model are denoted as (NI). Parameters included in tensor form are shown as (-).

\begin{tabular}{||ccccc||}
\hline \multicolumn{5}{|c|}{ Material constants } \\
\hline $\mathrm{Pt}$ & $\begin{array}{c}\text { Young's Modulus } \\
\mathrm{GPa}\end{array}$ & Poisson's Ratio & $\begin{array}{c}\text { Density } \\
\mathrm{kg} / \mathrm{m}^{3}\end{array}$ & $\begin{array}{c}\text { Damping } \\
\text { factor, } \eta\end{array}$ \\
$\mathrm{Au}$ & 168 & 0.38 & 21450 & $\mathrm{NI}$ \\
$\mathrm{PZT}$ & 78 & 0.44 & 19300 & $\mathrm{NI}$ \\
$\mathrm{SiO}_{2}$ & - & - & 7600 & 0.03 \\
$\mathrm{Si}_{3} \mathrm{~N}_{4}$ & 70 & 0.17 & 2650 & 0.0004 \\
$\mathrm{Si}$ & 270 & 0.24 & 3440 & $\mathrm{NI}$ \\
$\mathrm{Si}$ & - & - & 2330 & $\mathrm{NI}$ \\
\hline
\end{tabular}

simulation are listed in table 4.1 [32-35]. The elasticity matrix $C$ used for PZT and silicon in the simulation is given by [34]

$$
C=\left(\begin{array}{cccccc}
c_{11} & c_{12} & c_{21} & 0 & 0 & 0 \\
c_{12} & c_{11} & c_{21} & 0 & 0 & 0 \\
c_{21} & c_{21} & c_{33} & 0 & 0 & 0 \\
0 & 0 & 0 & c_{44} & 0 & 0 \\
0 & 0 & 0 & 0 & c_{44} & 0 \\
0 & 0 & 0 & 0 & 0 & c_{66}
\end{array}\right),
$$

where the coefficients for PZT are $c_{11}=134.8 \mathrm{GPa}, c_{12}=63.9 \mathrm{GPa}, c_{21}=68.1 \mathrm{GPa}$, $c_{33}=113.3 \mathrm{GPa}, c_{44}=22.2 \mathrm{GPa}$ and $c_{66}=33.4 \mathrm{GPa}$. For silicon there are only three independent coefficients $c_{11}=c_{33}=165.7 \mathrm{GPa}, c_{12}=c_{21}=63.9 \mathrm{GPa}$, and $c_{44}=c_{66}=51.0 \mathrm{GPa}$. The piezoelectric coupling tensor, $\boldsymbol{d}$, is given by [35]

$$
\mathbf{d}=\left(\begin{array}{cccccc}
0 & 0 & 0 & 0 & 440 & 0 \\
0 & 0 & 0 & 440 & 0 & 0 \\
-60 & -60 & 152 & 0 & 0 & 0
\end{array}\right) \times 10^{-12} \frac{\mathrm{C}}{\mathrm{N}},
$$

and the relative permittivity tensor, $\varepsilon^{T}$, is given by [35]

$$
\varepsilon^{T}=\left(\begin{array}{ccc}
990 & 0 & 0 \\
0 & 990 & 0 \\
0 & 0 & 450
\end{array}\right)
$$

where the superscript $T$ indicates that the relative permittivity tensor is measured under constant stress. 

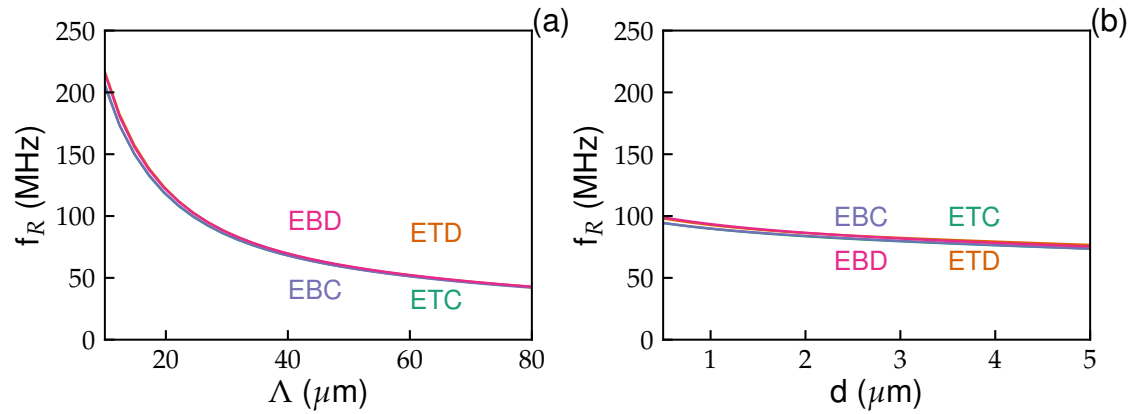

Figure 4.3: Resonant acoustic frequency, $f_{\mathrm{R}}$, of the fundamental Rayleigh wave for the four investigated IDT configurations (ETD, EBD, ETC, EBC, found in Fig. 4.2 ).The frequency is displayed as function of the period of the acoustic wave $\lambda_{\mathrm{R}}$, which is equal to the period $\Lambda$ of the IDT for a fixed PZT thickness $d=2 \mu \mathrm{m}$ (a), and as a function of the thickness $d$ of the PZT layer for an IDT period of $\Lambda=30 \mu \mathrm{m}(\mathrm{b})$.

\subsection{Results}

\subsubsection{Acoustic wave generation}

We are interested in MZI-based modulation with a maximum optical phase change in the interferometer arms. Therefore we consider a geometry where the SAW propagation direction is perpendicular to the optical axis of the waveguide, as in Figs. 4.1 and 4.2. The modulation frequency is taken as equal to the resonant frequency of the fundamental SAW. In this case, the acoustic wavelength, $\lambda_{\mathrm{R}}$, and frequency, $f_{\mathrm{R}}$, are equal to the period, $\Lambda$, and driving frequency of the IDT, respectively. The relation between $f_{\mathrm{R}}$ and $\Lambda_{\mathrm{R}}$ is given by

$$
f_{\mathrm{R}}=v_{\mathrm{R}} / \Lambda_{\mathrm{R}}
$$

where $v_{\mathrm{R}}$ is the phase velocity of the SAW. In general, $v_{\mathrm{R}}$ varies with $\Lambda, d$ and the different IDT configurations such that the modulation frequency of the optical wave will also vary with these parameters.

Figure 4.3(a) shows the acoustic frequency, $f_{\mathrm{R}}$, of the fundamental SAW as a function of the IDT period, $\Lambda$, for a fixed PZT layer thickness of $d=2.5 \mu \mathrm{m}$ in the four geometries shown in Fig. 4.2(c-f). Similarly, Fig. 4.3(b) shows $f_{\mathrm{R}}$ as a function of the PZT thickness, $d$, for a fixed IDT period of $\Lambda=30 \mu \mathrm{m}$ and the four geometries.

Figure 4.3(a) shows that the acoustic frequency monotonically decreases for all configurations as the IDT period increases. It can be seen that for large periods, all four configurations have approximately the same resonant acoustic frequency, while for 
shorter periods the configurations ETD and EBD posses a slightly higher resonant acoustic frequency compared to the configurations ETC and EBC.

Figure 4.3(b) shows as well that the frequency decreases monotonically for all configurations as the layer thickness increases. The configurations ETC and EBC show nearly identical resonant acoustic frequencies when the layer thickness is varied. For small layer thickness, the resonant acoustic frequency for configuration EBD is somewhat higher than that produced by configuration ETD. For larger layer thickness, the situation is reversed, i.e., the resonant acoustic frequency for configuration EBD is somewhat lower than that for configuration ETD.

The different resonant acoustic frequencies, found when the thickness of the layer is varied at constant IDT period, indicate that the sound velocity of the acoustic wave is affected by the amount of PZT material present. On the other hand, for a fixed geometry and varying only the IDT period, i.e., the period of the acoustic wave, we observe a strong increase in the resonant frequency when the period decreases (Fig. 4.3(a)), which is to be expected from the reciprocal relation between frequency and wavelength (c.f. Eq. 4.10). In summary, when the PZT layer is terminated with a conductive layer opposite to the IDT electrode, the resonant acoustic frequency is somewhat lower and is almost independent of the location of the IDT electrode (at PZT-air interface or $\mathrm{SiO}_{2}$-PZT interface), while when it is terminated with a dielectric layer, the resonant frequency is somewhat higher and is affected by the location of the IDT electrode.

In order to determine the change in effective refractive index of the fundamental optical mode that is induced by the SAW, the strain distribution generated by the SAW within the volume of the optical mode has to be calculated. A frequency domain analysis is performed to calculate the induced strain when a sinusoidal voltage is applied at a near resonant acoustic frequency to the IDT electrode. We note that with our symmetric unit cell (see Fig. 4.2(b)) the frequency domain analysis showed zero strain when excited at exactly the resonant frequency. However, when moving the frequency slightly off resonance, a nonzero strain is obtained. Adding acoustic damping to the cladding and piezo regions (see parameters in table 4.1), provides a physically realistic and numerically stable response.

A typical example of the strain distribution amplitude generated by the fundamental SAW when a voltage signal with an amplitude of $10 \mathrm{~V}$ is applied to the IDT electrode is shown in Fig. 4.4 for the configuration ETD with $\Lambda=15 \mu \mathrm{m}$ and $d=2 \mu \mathrm{m}$, which corresponds to modulation with $f_{\mathrm{R}}=150 \mathrm{MHz}$. In this figure, only the region of interest is shown, i.e., the region containing the waveguide's core and cladding. The origin of the coordinate system coincides with the center of the optical waveguide, and $y=8 \mu \mathrm{m}$ coincides with the $\mathrm{SiO}_{2}-\mathrm{PZT}$ interface. The $z$-axis (along which the optical mode propagates) points along the axis of the waveguide, normal to $x$ and $y$. Figure 4.4(a) shows the modulus of the induced strain in the $x$-direction, $\left|S_{x}\right|$, 

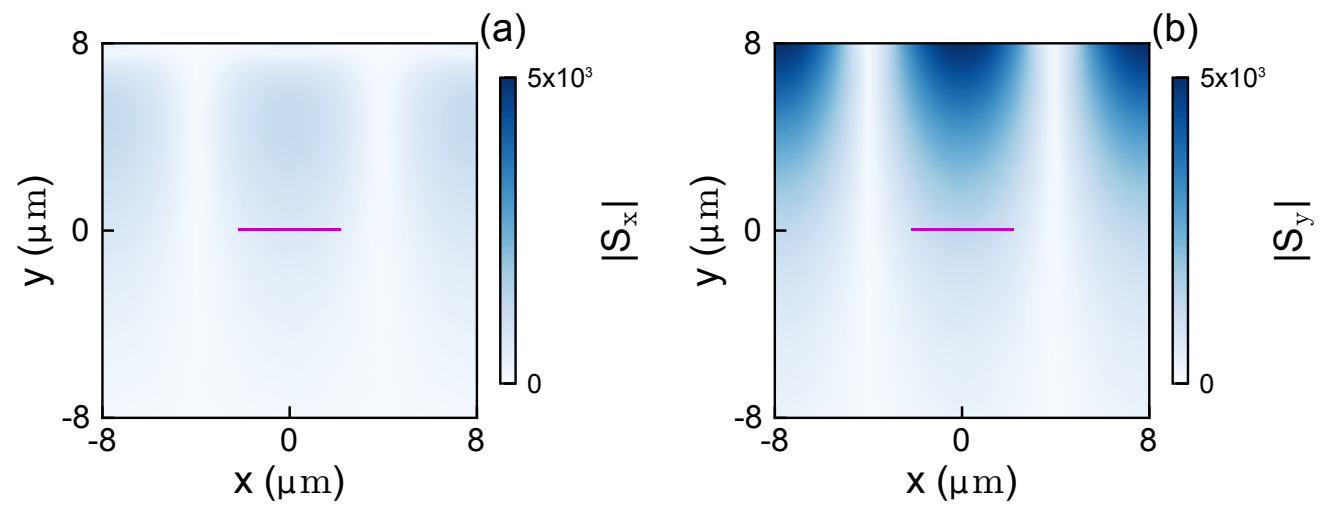

Figure 4.4: Strain distribution in the horizontal, $\left|S_{x}\right|$, and vertical, $\left|S_{y}\right|$, directions as generated by the fundamental Rayleigh wave when $\Lambda=$ $15 \mu \mathrm{m}$ and $d=2 \mu \mathrm{m}$. The $\mathrm{Si}_{3} \mathrm{~N}_{4}$ waveguide core is shown in purple at scale.

and Fig. 4.4(b) for strain in the $y$-direction, $\left|S_{y}\right|$. We observe that with increasing depth, $\left|S_{x}\right|$ grows from a near-zero value at $y \approx 8 \mu \mathrm{m}$, reaching a maximum before decreasing again towards $y=-8 \mu \mathrm{m}$. Inspecting $S_{x}$ within the PZT layer shows that $S_{x}$ changes sign as a function of depth and therefore there is a depth at which $S_{x}$ is zero. Note that for the parameters of Fig. 4.4, this depth is at the upper edge of the region plotted (near the $\mathrm{SiO}_{2}$-PZT interface) and moves to smaller $y$ values, i.e., larger depth below the PZT layer, when $\Lambda$ increases. In contrast to horizontal strain, the vertical strain, $S_{y}$, does not change sign and $S_{y}$ decreases monotonically with increasing depth, as seen in Fig. 4.4(b). Comparison of Figs. 4.4(a) and (b) shows that $\left|S_{y}\right|$ is the dominant strain at the location of the waveguide core and is typically a factor of 2 or more larger than $S_{x}$. This typical example shows that the SAWinduced strain easily extends to the core of the optical waveguide and, therefore, a good overlap between the induced strain and optical mode is expected.

Two of the other configurations (EBC and EBD) exhibit similar strain patterns (not separately shown), although with different strength. The remaining configuration, ETC displayed a different behavior in that the induced strain in the cladding region was significantly smaller (about six orders of magnitude) than for the other configurations. Apparently, the presence of the Pt layer between the PZT and $\mathrm{SiO}_{2}$, together with the presence of acoustic damping, results in a very poor coupling of the generated SAW into the cladding region. We note that by artificially turning off the acoustic damping, a similar behavior for the strain was obtained as for the other configurations. In the remainder of this chapter we will only consider the configurations ETD, EBC and EBD. 


\section{Maximizing strain}

In order to quantify how the strain can be maximized via variation of the thickness of the PZT layer and the period of the IDT electrode, we plot in Fig. 4.5(a) the dominant induced strain, $\left|S_{y}\right|$, as a function of the IDT period, $\Lambda$, for $d=2.5 \mu \mathrm{m}$ when a sinusoidal voltage with a near resonant frequency and an amplitude of $10 \mathrm{~V}$ is applied to the IDT for the three configurations considered. Similarly, Fig. 4.5(b) shows $\left|S_{y}\right|$ as a function of the layer thickness, $d$, for $\Lambda=25 \mu \mathrm{m}$.
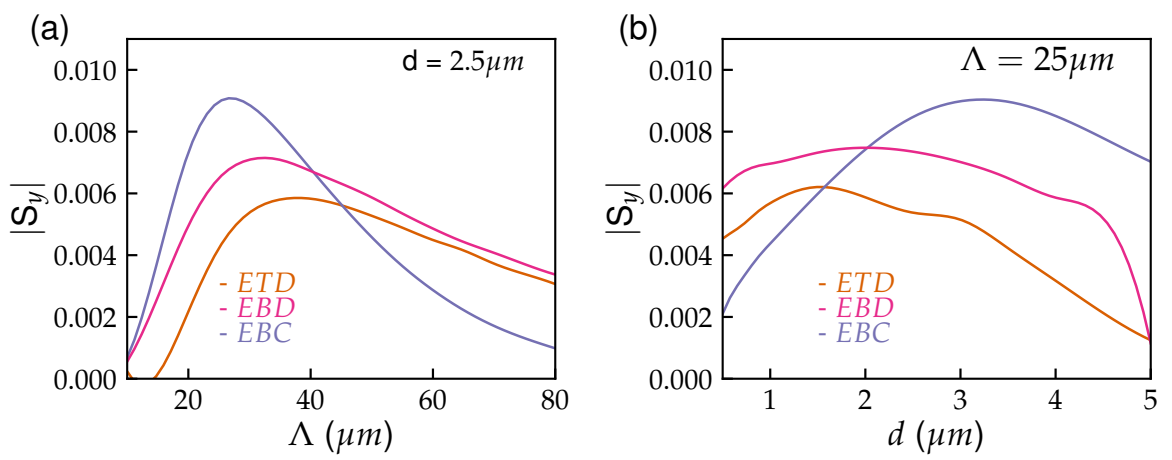

Figure 4.5: Strain distribution, $\left|S_{y}\right|$, in the vertical direction as produced by the fundamental Rayleigh SAW at the center of the optical waveguide as a function of IDT period, $\Lambda$ for $d=2.5 \mu \mathrm{m}$ (a) and as function of the layer thickness, $d$, for $\Lambda=25 \mu \mathrm{m}$. Only results for the configurations ETD,EBC and EBD are shown. A sinusoidal voltage with a near resonant frequency and an amplitude of $10 \mathrm{~V}$ is applied to the IDT electrode.

It can be seen in Fig. 4.5(a) that, with increasing IDT period, $\left|S_{y}\right|$ at the center of the optical waveguide increases before reaching a maximum. Increasing the IDT period further results in a decreasing $\left|S_{y}\right|$. The overall dependence of $\left|S_{y}\right|$ on $\Lambda$ is very similar for the three configurations, however, it can be seen that $\mathrm{EBC}$ produces the largest strain at the center of the waveguide for an IDT period that is smallest for the three configurations. Figure 4.5(a) also shows an increased decay of $\left|S_{y}\right|$ for periods $\Lambda \gtrsim 25 \mu \mathrm{m}$ for configuration $\mathrm{EBC}$ compared to the other two configurations. The behavior of $\left|S_{y}\right|$ at the center of the optical waveguide can be explained as follows.

As the IDT period increases, the acoustic wavelength of the SAW increases and the wave penetrates deeper into the structure towards the waveguide core. Consequently, the strain, $\left|S_{y}\right|$, at the location of the core is expected to increase. However, at the same time the volume corresponding to a single acoustic period increases as well. As the IDT electrode is powered by a constant voltage signal, this results in a decreasing amplitude of the sound wave and, consequently, the induced strain. The balance of these two effects is what gives rise to the maximum observed in 
Fig. 4.5(a). The different electric field distribution when a conductive layer is present opposite of the IDT electrode results in a more efficient excitation of the SAW wave for the optimum IDT period and PZT layer thickness combination at the cost of a faster decay in SAW excitation when the combinations moves away from the optimum.

A similar trend is visible in the dependence of $S_{y}$ on $d$ as shown in Fig. 4.5 (b) for the three configurations and $\Lambda=25 \mu \mathrm{m}$. Again, the configuration with the 100-nm gold layer on top $(\mathrm{EBC})$ provides the largest strain, $\left|S_{y}\right|$, at the waveguide core, followed by the configurations EBD and ETD. We also observe a maximum in the strain as function of the layer thickness, $d$, of the PZT layer, although the strain is near maximum over a wide range of thickness values $(1 \mu \mathrm{m}<d<3 \mu \mathrm{m})$ for configurations $\mathrm{EBD}$ and ETD. Figure 4.5(b) also shows that for configuration EBC the maximum strain is obtained at a larger PZT layer thickness than for the other two configurations. As expected, $\left|S_{y}\right|$ starts dropping to zero for small values of $d$, although much faster for $E B C$ than for the other two configurations. As the acoustic wavelength is not changed when the layer thickness is varied, these results show that there is an optimum thickness for the PZT layer to couple the electrical power supplied to the IDT into the SAW wave. This also maximizes the strain at the core of the waveguide, the value of which varies with the IDT period and depends on the configuration used. We attribute the larger maximum strain and higher sensitivity to the PZT layer thickness of configuration EBC to the 100-nm thick gold layer on top of the PZT layer, which affects the electric field distribution inside the PZT layer such that it produces the largest-amplitude SAW under optimum conditions.

In summary so far, we find that in order to maximize the acoustic modulation frequency, all configurations require a thin PZT layer and a small IDT period. Of the three configurations studied, ETD and EBD provide almost equal modulation frequencies for a given $d$ and $\Lambda$, which are somewhat higher than those for the two other configurations. However, the need to create maximum strain in the area of the optical mode requires an optimum IDT period with corresponding optimum PZT layer thickness, which will ultimately limit the maximum modulation frequency (via a decreasing index modulation) that can be realized.

\subsubsection{Modulation of the effective refractive index}

In this section we use the calculated strain induced in the core and cladding to obtain the change in effective refractive index for the fundamental mode propagating through the optical waveguide as function of $d$ and $\Lambda$ and for the three relevant configurations. 
As shown in the previous section, at modulation frequencies of the order of $100 \mathrm{MHz}$ the SAW-induced strain extends well into the cladding and should be able to cover the whole cross-sectional area occupied by the optical mode. This strain will lead to a change in the refractive index of the cladding and core via eqs. 4.2 and 4.3 , the strength of the coupling being set by the strain-optic coefficients. The strain-optic coefficients are not known for $\mathrm{Si}_{3} \mathrm{~N}_{4}$, however, due to our choice of a small core area and high aspect ratio, the influence of the core on the effective refractive index of the optical mode can be neglected. In the model we take the strain-optic coefficients for $\mathrm{Si}_{3} \mathrm{~N}_{4}$ equal to zero, to obtain a lower bound of the change in effective refractive index that can be realized. For $\mathrm{SiO}_{2}$ we take the strain-optic coefficients to be equal to $p_{11}=0.113$ and $p_{12}=0.252$ [36]. Due to the difference in strain in the $x$ and $y$-direction, the refractive index experienced by the mode is different for the quasi-TE and quasi-TM polarization [37]. To find the effective refractive index for the fundamental mode for the two polarization directions, we take the calculated strain and add the appropriate change in refractive index to the material refractive index [15] (using Eq. 4.2 for the quasi-TE and Eq. 4.3 for the quasi-TM polarization). Subsequently we use the eigenmode solver [32] to determine the propagation constant, $\beta$, and the spatial shape and field distribution for the fundamental quasi-TM mode. We then calculate the effective refractive index of this mode, $n_{\mathrm{eff}}=\frac{\beta \lambda}{2 \pi}$, and determine the difference, $\Delta n$, with the effective refractive index for the same mode in absence of a SAW.

Since the waveguide geometry we choose is meant for the visible and near-infrared applications [38], we selected an intermediate wavelength $(\lambda=840 \mathrm{~nm}$, as an example). Figure 4.6 shows the normalized intensity distribution of the fundamental guided mode with vertical polarization (quasi-TM mode), for a waveguide core area of $4.4 \times 0.03 \mu \mathrm{m}^{2}$ and using the same coordinate system as for Fig. 4.4. We observe that the mode is confined around the core and has negligible amplitude already being $5 \mu \mathrm{m}$ away from the core. Using the manufacturer supplies refractive indices for the core and cladding, the effective refractive index for this mode is found to be $n_{\text {eff }}=1.4646$, which is close to the refractive index of the $\mathrm{SiO}_{2}$ cladding. This confirms that most of the optical field is outside the $\mathrm{Si}_{3} \mathrm{~N}_{4}$ core and that approximating the influence of the strain in the core as negligible is justified. When the $\mathrm{SAW}$-induced strain is applied, the transverse shape of the intensity distribution as displayed in Fig. 4.6 is almost unaffected by the slight change in refractive index of the cladding material (in the order of $10^{-3}$ ), however, the longitudinal propagation constant is changed and, hence, the effective refractive index.

\section{Effective refractive index dependency on IDT periodicity}

In Fig. 4.7(a-c) we show the calculated relative change in effective refractive index, $\Delta n / n_{\text {eff }}$, for the fundamental mode with TM polarization as a function of the IDT 


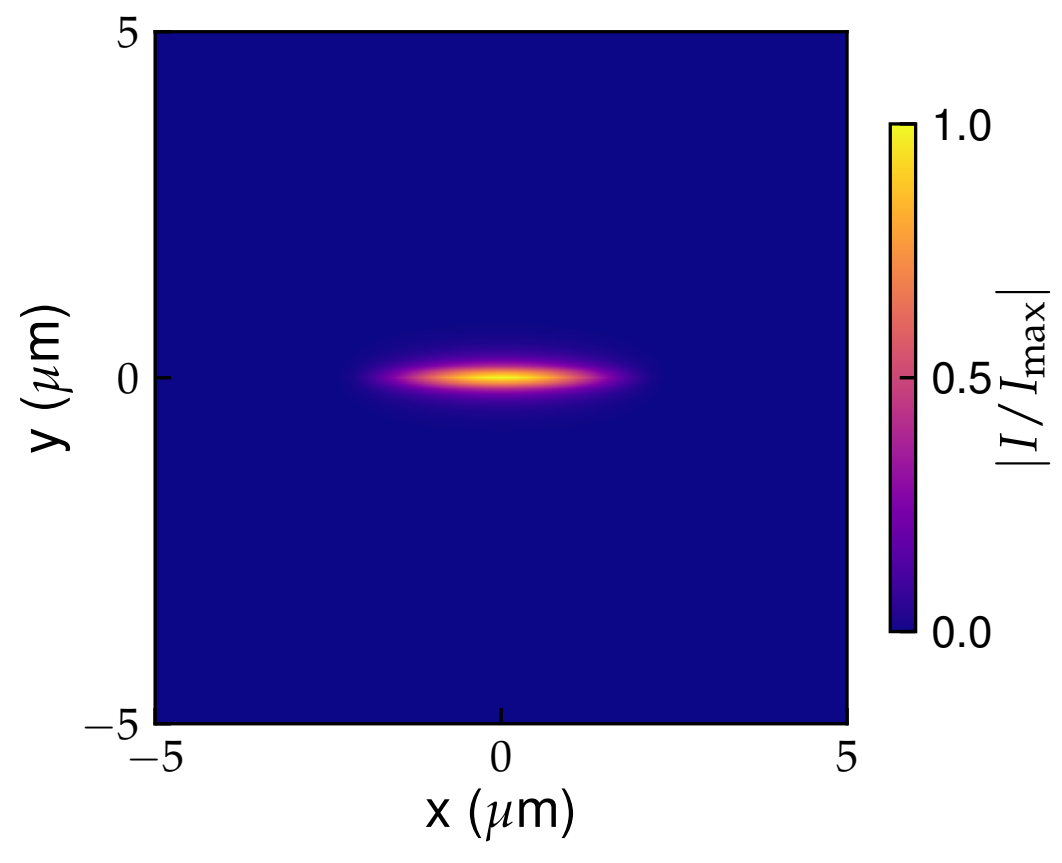

Figure 4.6: Normalized intensity distribution of the fundamental quasi-TM mode for a wavelength of $840 \mathrm{~nm}$. The $\mathrm{Si}_{3} \mathrm{~N}_{4}$ core with dimensions of $30 \mathrm{~nm}$ by $4.4 \mu \mathrm{m}$ is centered at the origin of the coordinate system. The drawing is to scale.

period, $\Lambda$, for the configurations $\operatorname{ETD}(\mathrm{a}), \operatorname{EBD}(\mathrm{b})$, and $\operatorname{EBC}(\mathrm{c})$, respectively. In each case, $\Delta n / n_{\text {eff }}$ is calculated for three different thicknesses of the PZT layer, $d=2.5$, 3 and $3.0 \mu \mathrm{m}$, while the voltage signal applied to the IDT period is maintained at a constant amplitude of $10 \mathrm{~V}$ with a configuration dependent near-resonant frequency (see Fig. 4.3). As expected from the calculations of the strain at the center of the waveguide core (see Fig. 4.4), Fig. 4.7 shows an initial increase in the relative change in effective refractive index with increasing period of the IDT before reaching a maximum and then starts to decrease when the period is further increased. This behavior is found for all three configurations investigated. However, the maximum $\Delta n / n_{\mathrm{eff}}$ is largest for the EBC configuration followed by the EBD and the ETD configurations, for which the maximum change in relative refractive index is smaller by a factor of 1.2 and 1.5, respectively. Furthermore, Fig. 4.7 shows that the maximum change in effective refractive index shifts to a lower value of $\Lambda$ when going from configuration 

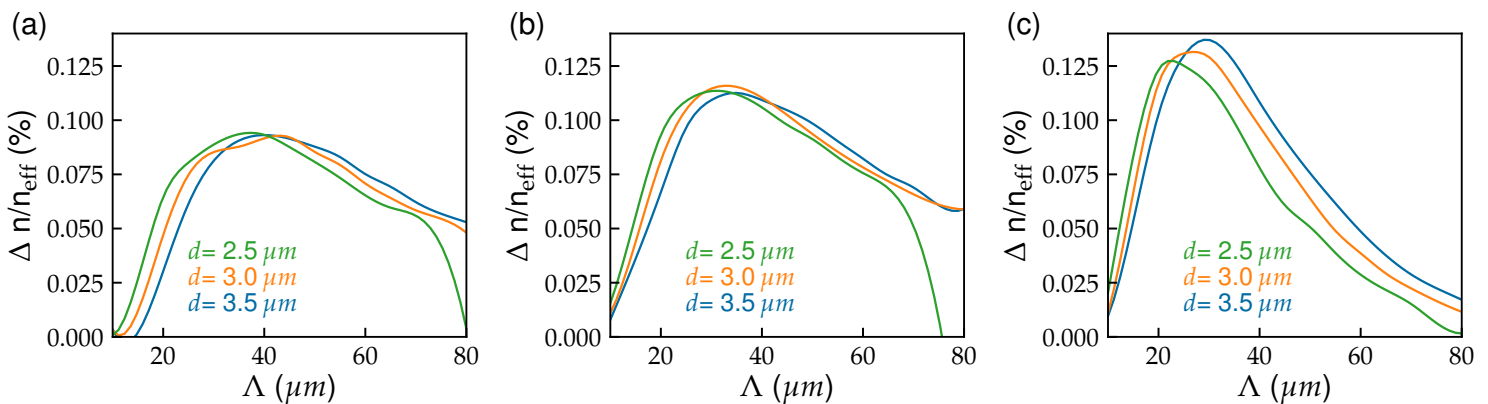

Figure 4.7: Relative change in the effective refractive index, $\Delta n / n_{\mathrm{eff}}$, as a function of IDT period, $\Lambda$, for three different PZT-layer thicknesses, $d=2.5,3.0$ and $3.5 \mu \mathrm{m}$, and for configuration ETD (a), EBD (b) and EBC (c). The vacuum wavelength is $\lambda=840 \mathrm{~nm}$ and the light is polarized in the y-direction (TM polarization).

ETD via EBD to EBC. According to Fig. 4.3, this leads to a higher modulation frequency, from around $90 \mathrm{MHz}$ to $100 \mathrm{MHz}$. Finally, we observe that the change in effective refractive index drops more quickly with increasing IDT period beyond the maximum for configuration EBC (Fig. 4.7(c)) compared to the other two configurations.

\section{Effective refractive index dependency on PZT thickness}

So far, we have only considered the variation with $\Lambda$ for a few fixed values of the PZT layer thickness. In Fig. 4.8(a-c) we show the calculated relative change in effective refractive index as a function of the PZT layer thickness, $d$, for the configurations $\operatorname{ETD}(\mathrm{a}), \operatorname{EBD}(\mathrm{b})$, and $\operatorname{EBC}(\mathrm{c})$, respectively. In each case, $\Delta n / n_{\text {eff }}$ is calculated for three different IDT periods, $\Lambda=25,30$ and $35 \mu \mathrm{m}$ and the remaining parameters are as for Fig. 4.7. With the IDT electrode on top (ETD), i.e., with the electrode at the PZT-air interface, we find that $\Delta n / n_{\text {eff }}$ is almost constant for $d<3 \mu \mathrm{m}$ for each of the three IDT periods investigated. For larger values of $d, \Delta n / n_{\text {eff }}$ starts to differ between the three configurations (Fig. 4.8(a)), with the lowest IDT period of $\Lambda=25 \mu \mathrm{m}$ showing a faster drop in $\Delta n / n_{\text {eff }}$ than for the other two IDT period investigated. For the case of the IDT electrode placed at the bottom of the PZT layer and no conductive layer placed in the opposite side (EBD), Fig. 4.8(b) shows that $\Delta n / n_{\text {eff }}$ is almost independent of $d$ for the three IDT periods investigated. A decrease in $\Delta n / n_{\text {eff }}$ is found only for very small $(<1 \mu \mathrm{m})$ or very large $(>4 \mu \mathrm{m})$ thickness of the PZT layer. On the other hand, with the IDT electrode at the bottom and a 100-nm conductive gold layer at the opposite site of the PZT layer ( $\mathrm{EBC})$, Fig. 4.8(c) shows a strong variation of $\Delta n / n_{\text {eff }}$ with $d$ for all periods investigated. The optimum layer thickness for maximum $\Delta n / n_{\mathrm{eff}}$ is between 3 and $4 \mu \mathrm{m}$, depending on the IDT period. 

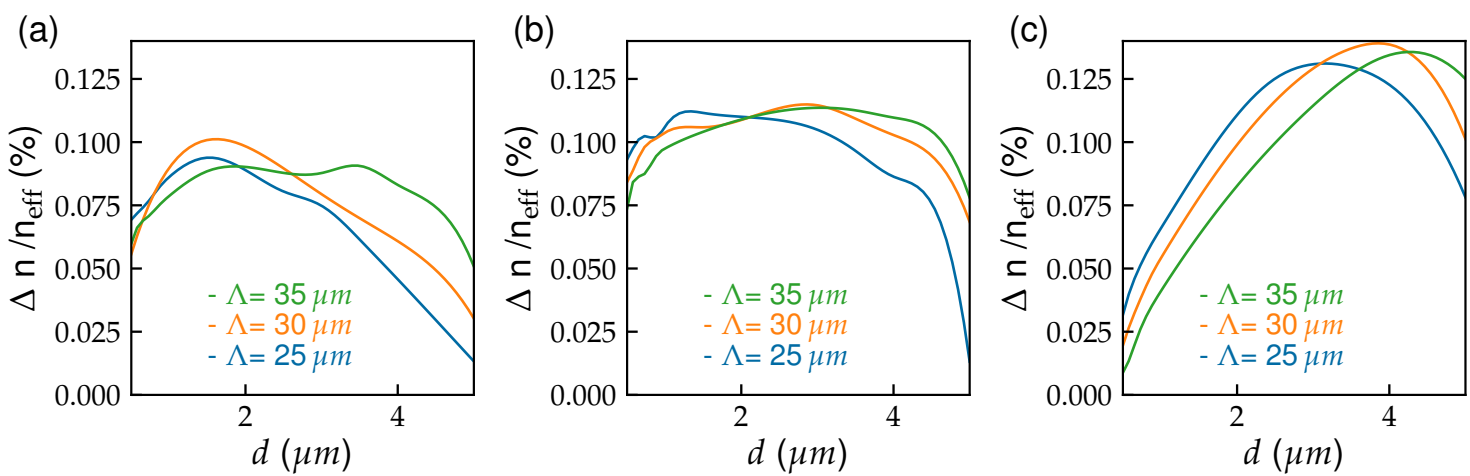

Figure 4.8: Relative change in the effective refractive index, $\Delta n / n_{\mathrm{eff}}$, as a function thickness of the PZT layer, $d$ for three different IDT periods, $\Lambda=25,30$ and $35 \mu \mathrm{m}$ for configuration ETD (a), EBD (b) and EBC (c). The vacuum wavelength is $\lambda=840 \mathrm{~nm}$ and the light is polarized in the $\mathrm{y}$-direction (TM polarization).

The largest $\Delta n / n_{\text {eff }}$ of $\sim 0.14 \%$ is obtained with this configuration for $\Lambda \approx 30 \mu \mathrm{m}$ and $d \approx 4 \mu \mathrm{m}$. This corresponds to an absolute change in index of $\Delta \mathrm{n}=2.0 \times 10^{-3}$. Both Figs. 4.7 and 4.8 show that the induced change in effective refractive index depends on (i) the location of the IDT electrode, (ii) its period, (iii) the thickness of the PZT layer and (iv) whether there is a thin conductive layer opposite the IDT electrode or not. To find the maximum value for $\Delta n / n_{\text {eff }}$ requires a two-dimensional scan over the IDT period and PZT layer thickness for each of the configurations. Although we have not fully scanned the complete parameter space, Figs. 4.7 and 4.8 indicate that the scans presented in these figures should be close to or even contain the optimum combination of $\Lambda$ and $d$ to achieve a maximum change in the effective refractive index for each of the configurations investigated.

In summary, having an IDT electrode on top of the PZT layer with a 100-nm thick Pt layer at the $\mathrm{SiO}_{2}-\mathrm{PZT}$ interface (ETC) results in a extremely weak SAW in the cladding region. This configuration was therefore not studied further. Having instead a very thin dielectric seed layer at the $\mathrm{SiO}_{2}$-PZT interface (ETD) results in a SAW that penetrates well into the cladding region, however the magnitude of the induced strain and hence the of the maximum relative change in the effective refractive index of $\sim 0.1 \%$ is smallest of the remaining configurations. Optimum values for the IDT period and PZT layer thickness are $25 \mu \mathrm{m}<\Lambda<40 \mu \mathrm{m}$ and $1 \mu \mathrm{m}<d<2 \mu \mathrm{m}$, respectively, depending on the configuration. Moving the IDT electrode to the $\mathrm{SiO}_{2}$ PZT interface and having no opposite conductive layer (EBD) generates a maximum relative change in effective refractive index of $\sim 0.11 \%$ for a wide range of IDT periods $(25 \mu \mathrm{m}<\Lambda<40 \mu \mathrm{m})$ and thicknesses of the PZT layer $(1 \mu \mathrm{m}<d<4 \mu \mathrm{m})$. Finally, terminating the $\mathrm{SiO}_{2}$-air interface with a 100-nm gold layer (EBC) results in 
a configuration that generates the largest relative change in effective refractive index of $\sim 0.14 \%$, although with the smallest parameter range of $20 \mu \mathrm{m}<\Lambda<30 \mu \mathrm{m}$ and $3.5 \mu \mathrm{m}<d<4 \mu \mathrm{m}$. With the additional gold layer at the $\mathrm{SiO}_{2}$-air interface, the electric field provided via the IDT electrode is confined to the PZT layer and this produces a SAW wave that generates the largest strain at the location of the waveguide core, though with a stronger dependence on the IDT period and PZT layer thickness. Although this configuration and the required thickness of the PZT layer results in a slightly lower resonant acoustic frequency (c.f. Fig. 4.3), and hence the modulation frequency, the larger induced change in effective refractive index makes this the favorable configuration of the four investigated.

\section{Optimized design and geometry}

Now that we have calculated the maximum value for $\Delta n / n_{\text {eff }}$ provided by the optimal configuration, which is $0.14 \%$, we use Eq. 4.6 to calculate the required length of the arms of a balanced Mach-Zehnder interferometer to obtain complete light modulation at the acoustic resonance frequency.

For a vacuum wavelength of $\lambda=840 \mathrm{~nm}$, we find $L=103 \mu \mathrm{m}$ using $n_{\text {eff }}=1.464$ for the quasi-TM polarized light. The voltage applied to the IDT has an amplitude of $10 \mathrm{~V}$. Due to the linearity of the strain with applied voltage expected in this regime of small strain and index modulation, obtaining full light modulation at a shorter wavelength, say half the used wavelength $(420 \mathrm{~nm})$ would require driving the IDT only at half the voltage $(\sim 5 \mathrm{~V})$. In contrast, modulating light with a longer wavelength, say at telecommunication wavelengths $(1550 \mathrm{~nm})$, would almost double the required driving voltage $(\sim 18.5 \mathrm{~V})$. Nevertheless, both values are well within the expected operating range that may extend to voltage amplitudes of $50 \mathrm{~V}$ or more before breakdown occurs $[19,29,35]$. This means that depending on the selected voltage or wavelength even a smaller arm length than $103 \mu \mathrm{m}$ might be sufficient to obtain full light modulation.

\subsection{Summary and Conclusions}

In this work we investigated the use of a Rayleigh-type surface acoustic waves (SAWs) to modulate the effective refractive index of an optical mode guided by a buried $\mathrm{Si}_{3} \mathrm{~N}_{4}$ waveguide core in a $\mathrm{SiO}_{2}$ cladding. We considered that the acoustic waves are excited in a PZT piezo-electric film layer deposited on top of the waveguide cladding via interdigitized electrodes, at a frequency in the order of $100 \mathrm{MHz}$. 
Considering a balanced Mach-Zehnder interferometer, the modulation of the effective refractive index can be used to obtain full, i.e., $100 \%$-modulation of the light power and amplitude, at the acoustic frequency. The optical waveguide considered here consists of a $\mathrm{Si}_{3} \mathrm{~N}_{4}$ core, with dimensions of $4.4 \mu \mathrm{m}$ by $30 \mathrm{~nm}$, buried in a $\mathrm{SiO}_{2}$ cladding $8 \mu \mathrm{m}$ below the surface, which is typical for this low-loss photonic platform. The SAWs generated by the thin PZT layer is guided in the interface between the PZT and the cladding, while its evanescent strain field extends towards depths that include the waveguide core. The strain induced by the SAW results in a change of the effective refractive index of the waveguide via the strain-optic effect.

We find that of four investigated IDT-PZT arrangements, the combination of IDT electrode at the interface $\mathrm{SiO}_{2}$-PZT with a counter-electrode at the PZT-air interface (configuration $\mathrm{EBC}$ ) is the most efficient in generating strain in the cross sectional area of the optical mode. This results in a maximum relative change in effective refractive index for the fundamental waveguide mode of $\Delta n / n_{\text {eff }}=0.14 \%$ for a wavelength in the middle of the working range of this waveguide, here taken as $\lambda=840 \mathrm{~nm}$, with quasi-TM polarization. The maximum modulation is obtained near-resonance at a driving voltage of $10 \mathrm{~V}$ for an IDT electrode periodicity $\Lambda=$ $30 \mu \mathrm{m}$ with a PZT thickness $d=4 \mu \mathrm{m}$.

For the maximum relative change in refractive index, the arm length required in a balanced Mach-Zehnder interferometer is $103 \mu \mathrm{m}$, with $100 \%$ light modulation driven with $10 \mathrm{~V}$ at a frequency of $90 \mathrm{MHz}$. We note that this frequency is larger by at about five orders of magnitude compared to thermo-optic intensity modulators and about two orders of magnitude compared to a stress-optic intensity modulator where stress is induced via a single electrode [18].

We note that also the required interaction length (for a MZI) is shorter, by a factor about five to ten, than what is typically used in thermally operated MZI (500 $\mu \mathrm{m})$ and by a factor of 80 compared to proximity strain-optic modulation [18]. As a parallel route for optimization, IDT electrodes might be configured to generate a focused SAW [39] to increase the strain in the region of the optical mode. An additional variation would be meandering the optical waveguide though the SAW field for shortening of the required overlap length with the transverse SAW field dimension. Also, the cladding height above the core might be reduced by one third without affecting notably the optical propagation loss, because the optical mode with our example wavelength, and also for even shorter wavelengths as in [18] does not extend too far outside the core (c.f. Fig. 4.6). Another advantage of using a SAW to drive a Mach-Zehnder interferometer is that it can coherently drive multiple interferometers located suitably next to each other for providing a stable phasing relative to each other. This is if interest, e.g., for low-loss phase modulators that form optical isolators based on acoustic waves [40,41]. 


\section{References}

1. Bauters, J. F., Heck, M. J. R., John, D., Dai, D., Tien, M.-C., Barton, J. S., Leinse, A., Heideman, R. G., Blumenthal, D. J. \& Bowers, J. E. Ultra-low-loss high-aspect-ratio Si3N4 waveguides. Opt. Express 19, 3163-3174 (2011).

2. Wörhoff, K., Heideman, R., Leinse, A. \& Hoekman, M. TriPleX: A versatile dielectric photonic platform. Adv. Opt. Techn. 4, 189-207 (2015).

3. Heck, M. J. R., Bauters, J. F., Davenport, M. L., Spencer, D. T. \& Bowers, J. E. Ultra-low loss waveguide platform and its integration with silicon photonics. Laser Photonics Rev. 8, 667-686 (2014).

4. Epping, J. P. et al. On-chip visible-to-infrared supercontinuum generation with more than $495 \mathrm{THz}$ spectral bandwidth. Opt. Express 23, 19596-19604 (2015).

5. Nguyen, V. D., Weiss, N., Beeker, W., Hoekman, M., Leinse, A., Heideman, R. G., van Leeuwen, T. G. \& Kalkman, J. Integrated-optics-based swept-source optical coherence tomography. Opt. lett. 37, 4820-4822 (2012).

6. Zinoviev, K., Carrascosa, L. G., Sánchez del Río, J., Sepúlveda, B., Domínguez, C. \& Lechuga, L. M. Silicon Photonic Biosensors for Lab-on-a-Chip Applications. Advances in Optical Technologies 2008, 383927 (2008).

7. Roeloffzen, C. G. H., Zhuang, L., Taddei, C., Leinse, A., Heideman, R. G., van Dijk, P. W. L., Oldenbeuving, R. M., Marpaung, D. A. I., Burla, M. \& Boller, K.-J. Silicon nitride microwave photonic circuits. Opt. Express 21, 22937-22961 (2013).

8. Marpaung, D., Roeloffzen, C., Heideman, R., Leinse, A., Sales, S. \& Capmany, J. Integrated microwave photonics. Laser Photon. Rev. 7, 506-538 (2013).

9. Oldenbeuving, R. M., Klein, E. J., Offerhaus, H. L., Lee, C. J., Song, H. \& Boller, K.-J. $25 \mathrm{kHz}$ narrow spectral bandwidth of a wavelength tunable diode laser with a short waveguide-based external cavity. Laser Phys. Lett. 10, 015804 (2013).

10. Halir, R., Okawachi, Y., Levy, J. S., Foster, M. A., Lipson, M. \& Gaeta, A. L. Ultrabroadband supercontinuum generation in a CMOS-compatible platform. Opt. Lett. 37, 16851687 (2012).

11. Zhuang, L. et al. On-chip microwave photonic beamformer circuits operating with phase modulation and direct detection. Opt. Express 22, 17079-17091 (2014).

12. Xiong, C. et al. Compact and reconfigurable silicon nitride time-bin entanglement circuit. Optica 2, 724-727 (2015).

13. Zhuang, L., Roeloffzen, C. G. H., Hoekman, M., Boller, K.-J. \& Lowery, A. J. Programmable photonic signal processor chip for radiofrequency applications. Optica 2, 854-859 (2015).

14. Ovvyan, A., Gruhler, N., Ferrari, S. \& Pernice, W. Cascaded Mach-Zehnder interferometer tunable filters. J. Opt. 18, 064011 (2016).

15. Korpel, A. Acousto-Optics, Second Edition (CRC Press, 1996).

16. Yariv, A. \& Yeh, P. Photonics: Optical Electronics in Modern Communications (Oxford University Press, Inc., New York, NY, USA, 2007).

17. Schriever, C., Bohley, C., Schilling, J. \& Wehrspohn, R. B. Strained Silicon Photonics. Materialss 5, 889-908 (2012). 
18. Hosseini, N., Dekker, R., Hoekman, M., Dekkers, M., Bos, J., Leinse, A. \& Heideman, R. Stress-optic modulator in TriPleX platform using a piezoelectric lead zirconate titanate (PZT) thin film. Opt. Express 23, 14018-14026 (2015).

19. De Lima Jr., M. M., Beck, M., Hey, R. \& Santos, P. V. Compact Mach-Zehnder acoustooptic modulator. Appl. Phys. Lett. 89, 121104 (2006).

20. Tadesse, S. A. \& Li, M. Sub-optical wavelength acoustic wave modulation of integrated photonic resonators at microwave frequencies. Nat. Commun. 5, 5402 (2014).

21. Tadesse, S. A., Li, H., Liu, Q. \& Li, M. Acousto-optic modulation of a photonic crystal nanocavity with Lamb waves in microwave K band. Appl. Phys. Lett. 107, 201113 (2015).

22. Rayleigh, L. On waves propagated along the plane surface of an elastic solid. s1-17, 4-11 (1885).

23. Lima, M. M. d. \& Santos, P. V. Modulation of photonic structures by surface acoustic waves. Reports on Progress in Physics 68, 1639-1701 (2005).

24. Kay, H. Electrostriction. Rep. Prog. Phys. 18, 230 (1955).

25. Sundar, V. \& Newnham, R. E. Electrostriction and polarization. Ferroelectrics 135, 431446 (1992).

26. Boyd, R. W. Nonlinear Optics (Academic Press, 2013).

27. Borrelli, N. F. \& Miller, R. A. Determination of the Individual Strain-Optic Coefficients of Glass by an Ultrasonic Technique. Appl. Opt. 7, 745-750 (1968).

28. Telford, W., Geldart, L. \& Sheriff, R. Applied Geophysics (Cambridge University Press, 1990).

29. Hashimoto, K. Surface aucoustic devices in telecommunications: modelling and simulation (Springer-Verlag, Berlin; Germany, 2000).

30. Saito, Y., Takao, H., Tani, T., Nonoyama, T., Takatori, K., Homma, T., Nagaya, T. \& Nakamura, M. Lead-free piezoceramics. Nature 432, 84-87 (2004).

31. Fukushima, J., Kodaira, K. \& Matsushita, T. Preparation of ferroelectric PZT films by thermal decomposition of organometallic compounds. Journal of Materials Science 19, 595-598 (1984).

32. COMSOL Multiphysics $囚$ version 5.0. www.comsol.com, 2014.

33. Lazan, B. J. Damping of materials and members in structural mechanics 340 pp. (Pergamon Press, 1968).

34. Zhang, L., Barrett, R., Cloetens, P., Detlefs, C. \& Sanchez del Rioa, M. Anisotropic elasticity of silicon and its application to the modelling of X-ray optics. J. Synchrotron Radiat. 21, 507-517 (2014).

35. http://www.efunda.com/ 2017.

36. Bertholds, A. \& Dandliker, R. Determination of the individual strain-optic coefficients in single-mode optical fibres. J. Lightwave Technol. 6, 17-20 (1988).

37. Fallahkhair, A. B., Li, K. S. \& Murphy, T. E. Vector Finite Difference Modesolver for Anisotropic Dielectric Waveguides. J. Lightwave Technol. 26, 1423-1431 (2008).

38. Haglund, E. P., Kumari, S., Gustavsson, J. S., Haglund, E., Roelkens, G., Baets, R. G. \& Larsson, A. Hybrid vertical-cavity laser integration on silicon in. Vertical-Cavity SurfaceEmitting Lasers XXI (International Society for Optics and Photonics, 2017), 101220H.

39. Collins, D. J., Neild, A. \& Ai, Y. Highly focused high-frequency travelling surface acoustic waves (SAW) for rapid particle sorting. Lab. Chip 16, 471 (2016). 
40. Hendrickson, S. M., Foster, A. C., Camacho, R. M. \& Clader, B. D. Integrated nonlinear photonics: emerging applications and ongoing challenges. J. Opt. Soc. Am. B 31, $3193-$ 3203 (2014).

41. Shi, Y., Cerjan, A. \& Fan, S. Acousto-optic finite-difference frequency-domain algorithm for first-principles simulations of on-chip acousto-optic devices. APL Photonics 2, 020801 (2017). 



\section{Chapter 5}

\section{Second-harmonic generation}

We report the observation of second-harmonic generation (SHG) in stoichiometric silicon nitride waveguides grown via low-pressure chemical vapor deposition (LPCVD). Quasi-rectangular waveguides with a large cross section were used, with a height of $1 \mu \mathrm{m}$ and various different widths, from 0.6 to $1.2 \mu \mathrm{m}$, and with various lengths from 22 to $74 \mathrm{~mm}$. Using a modelocked laser delivering 6-ps pulses at $1064 \mathrm{~nm}$ wavelength with a repetition rate of $20 \mathrm{MHz}, 15 \%$ of the incoming power was coupled through the waveguide, making maximum average powers of up to $15 \mathrm{~mW}$ available in the waveguide depending on the waveguide cross section. Second-harmonic output was observed with a delay of minutes to several hours after the initial turn-on of pump radiation, after which it grew within tens of seconds up to minutes to a steady state, with the shortest delay and faster growth at the highest input power. After this first, initial build-up, the second-harmonic became generated instantly with each new turn-on of the pump laser power. Phase matching was found to be present independent of the used waveguide width, although the latter changes the fundamental and secondharmonic phase velocities. We address the presence of a second-order nonlinearity and phase matching, involving an initial, power-dependent buildup, to the coherent photogalvanic effect. The effect, via the third-order nonlinearity and multiphoton absorption leads to a spatially patterned charge separation, which generates a spatially periodic, semi-permanent, DC-fieldinduced second-order susceptibility with a period that is appropriate for quasi-phase matching. The maximum measured second-harmonic conversion efficiency amounts to $0.4 \%$ in a waveguide with $0.9 \times 1 \mu \mathrm{m}^{2}$ cross section and $36 \mathrm{~mm}$ length, corresponding to $53 \mu \mathrm{W}$ at $532 \mathrm{~nm}$ with $13 \mathrm{~mW}$ of IR input coupled into the waveguide. The according $\chi^{(2)}$-susceptibility amounts to $3.7 \mathrm{pm} / \mathrm{V}$, as retrieved from the measured conversion efficiency. 


\subsection{Introduction}

Photonic integrated circuits (PICs) realized with various different material platforms, e.g., SOI (silicon on insulator) [1, 2], InP [3, 4], polymers [3], $\mathrm{SiO}_{2}[2,3]$ and $\mathrm{SiN}[5,6]$, have gained a tremendously growing importance in modern photonic technologies. This is due to a huge variety of emerging applications in highthroughput communications [7], optical sensing [8] and the life sciences, the latter, specifically, when involving visible light $[9,10]$. Semiconductor waveguide platforms offer the strongest first-order optical interactions, such as required for light generation, amplification or photo detection. Dielectric waveguide platforms, on the other hand, provide lowest propagation loss and tolerate high intensities, thereby enabling various types of optical functionalities via nonlinear optical interactions.

A most prominent example is photonic circuits fabricated from stoichiometric $\mathrm{Si}_{3} \mathrm{~N}_{4}$ grown via low-pressure chemical vapor deposition (LPCVD), embedded in a $\mathrm{SiO}_{2}$ cladding. This platform offers a unique combination of adjustable properties, i.e., record-low propagation loss $(<0.001 \mathrm{~dB} / \mathrm{cm})$ [5] such as for high-Q resonators [11], a wide spectral range of optical transparency (from about $310 \mathrm{~nm}$ throughout the entire visible spectrum up to $5.5 \mu \mathrm{m}$ [12]), and a high index contrast to achieve tight mode confinement via fabrication of relatively thick waveguide cores [13]. This platform has also reached a considerable degree of maturity, allowing two-dimensional tapering [14] for low-loss fiber coupling or realizing hybrid lasers [15, 16]. The platform supports also a wide range of optical functionalities [11, 17] by offering various different core cross sections for waveguide dispersion engineering, and offers a highly reproducible material dispersion [13, 14]. Regarding third-order nonlinearities, the recent demonstration of the broadest-ever optical spectrum generated on a chip, $495 \mathrm{THz}$ when pumped at $1064 \mathrm{~nm}$ [18], shifted more towards the midinfrared when pumped at $1550 \mathrm{~nm}$, while still maintaining a bandwidth of $453 \mathrm{THz}$ (Chapter 3) [19]. This has opened a wide prospective towards four-wave mixing [20], frequency comb generation [21] and all-optical switching [22].

Additional functionalities that can be used with lower field strengths would become accessible if this platform would as well provide a second-order nonlinear response, for instance for electro-optic modulation, second-harmonic generation (SHG), or parametric down-conversion. Such nonlinearity, having as its signature a non-zero second-order susceptibility, is also of interest for generating quantum correlated photon pairs directly within reconfigurable time-bin entanglement circuits [23].

A fundamental precondition for making use of such second-order nonlinearity is, however, that the material provides a non-inversion symmetric structure. With the discussed silicon nitride platform, this is a problem because the involved materials, $\mathrm{Si}_{3} \mathrm{~N}_{4}$ and $\mathrm{SiO}_{2}$, are amorphous and thus inversion symmetric. On the other 
hand, there have been two reports on second-order response in related amorphous SiN-type materials. Specifically, second-harmonic generation has been observed in $\mathrm{SiN}$ waveguides (fabricated with plasma enhanced chemical vapor deposition at lower temperatures [24, 25]), and in silicon-enriched SiN films (fabricated with RF sputtering [26]). Such differences are important to note because both the stoichiometric ratio as well as the type of fabrication process have a strong influence on the size of the optical bandgap, the propagation losses and the third-order nonlinear response [27]. The stoichiometric, low-loss $\mathrm{Si}_{3} \mathrm{~N}_{4}$ material waveguides described above have not been investigated so far for their second-order nonlinear response, thus leaving open whether functionalities based on a non-zero $\chi^{(2)}$ susceptibility can be realized.

Here we present the first observation of a second-order response in LPCVD-grown, stoichiometric $\mathrm{Si}_{3} \mathrm{~N}_{4}$ waveguides, using second-harmonic generation. Employing a mode-locked laser at a wavelength of $1064 \mathrm{~nm}$ delivering 6.2 ps pulses and coupling an average power of $13 \mathrm{~mW}$ into a waveguide with $0.9 \times 1 \mu \mathrm{m}^{2}$ cross-section and $36 \mathrm{~mm}$ length, a second-harmonic (SH) output of $53 \mu \mathrm{W}$ was reached, which corresponds to a conversion of $0.4 \%$, with an effective second-order nonlinear susceptibility of $3.7 \mathrm{pm} / \mathrm{V}$. We observed that in order to generate a SH output, an optical initialization process is required, similar to what had been observed earlier in glass fibers $[28,29]$. The process involves exposing the waveguides with the in-coupled IR pulses at $\mathrm{mW}$ power levels over time intervals between several minutes to hours, depending on the infrared power.

\subsection{Experimental procedure and results}

In our experimental approach we aimed on demonstrating second-harmonic generation based on modal phase matching [30]. This requires the identification of proper waveguide dimensions that provide the same effective refractive index (waveguide index) for propagation at the fundamental and second-harmonic frequencies. To calculate the effective refractive index dispersion, $n_{\text {eff }}(\omega)$, as function of the width and height of the waveguide core we use a fully vectorial finite-element mode solver [31] with the Sellmeier dispersion data given by Eqs. 2.7 and 2.8, for the wavelength range of the available pump laser (around $1.064 \mu \mathrm{m}$ wavelength) and its second-harmonic (around $532 \mathrm{~nm}$ ). For maximizing the waveguide-internal intensity via a strong confinement, enlarged core cross-sections are considered with a height and width around $1 \mu \mathrm{m}$. The two-dimensional step-index profile used for the calculations is based on the actual waveguide shape available from SEM images [13]. 
Figure 5.1 gives an overview of the calculated effective index, $n_{\mathrm{eff}}, v s$. the waveguide width, $w$, for a constant waveguide height, $h$, of $1 \mathrm{um}$ for two polarizations. The effective refractive index for the fundamental guided mode of the infrared (IR) pump light (labeled $E_{11}$ ), at a vacuum wavelength of $1064 \mathrm{~nm}$, is shown as red curves. The effective refractive index of the next-higher transverse modes, $\mathrm{E}_{i j}$, of the according second-harmonic at $532 \mathrm{~nm}$ wavelength is shown as green and blue curves, for quasi- $x$ (horizontally) and quasi- $y$ (vertically) polarized light (Figs. 5.1(a) and (b), respectively). It can be seen that modal phase matching involving the $\mathrm{E}_{11}$ mode and a given polarization is expected only for a single, specific waveguide width where the IR dispersion curve crosses a specific SH dispersion (green) curve as indicated with a black circle. According to the calculations, phase matching is expected with a waveguide width of about $w=0.65 \mu \mathrm{m}$ for the horizontally polarized transverse mode $\mathrm{E}_{13}$, and about $w=0.68 \mu \mathrm{m}$ for the vertically polarized transverse mode $\mathrm{E}_{21}$.

(a)

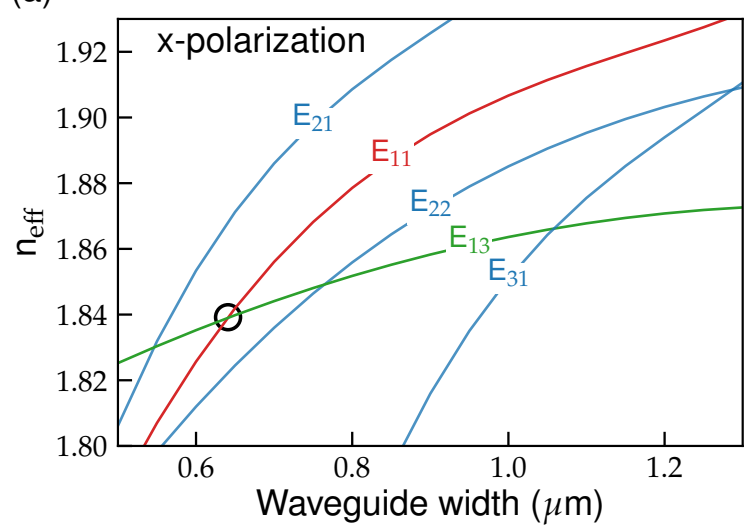

(b)

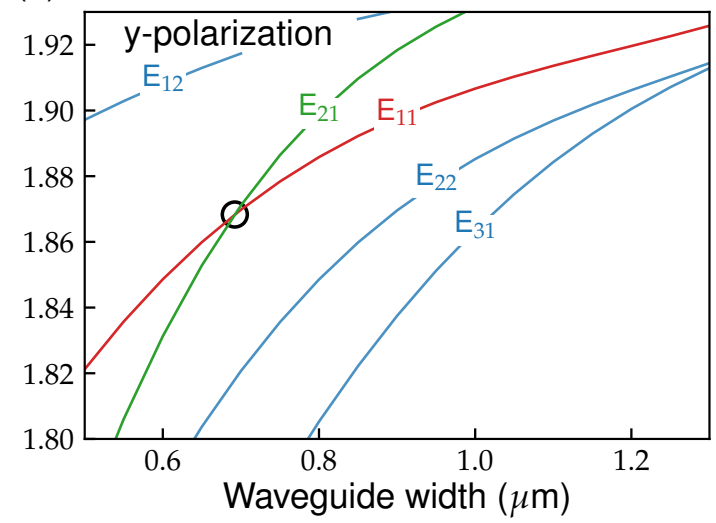

Figure 5.1: (a) Calculated effective refractive index vs. waveguide width, $w$, for the IR fundamental mode $\left(\mathrm{E}_{11}\right)$ with quasi-horizontal polarization (red curve), and for various transverse modes at 532 with quasi-horizontal polarization. The waveguide height is fixed at $1 \mu \mathrm{m}$. (b) Analogous calculation of dispersion for quasi-vertically polarized modes. The black circles indicate where modal phase matching is expected, namely with $w=0.65 \mu \mathrm{m}$ or $0.68 \mu \mathrm{m}$.

Figure 5.2 shows the experimental setup employing a mode-locked Yb-fiber laser operating at a wavelength of $1064 \mathrm{~nm}$ with a pulse duration of $6.2 \mathrm{ps}$ and a repetition rate of $20 \mathrm{MHz}$ (Toptica, PicoFYb 1064). The power of the laser and its polarization was controlled via two half-wave plates and a polarizing beam splitter. To maximize the input coupling, the round cross-section provided by the laser in a collimated beam was reduced from $4 \mathrm{~mm}$ to $2.5 \mathrm{~mm}$ with a telescope arrangement. Due to the high confinement of IR light in the waveguide, with a calculated effective mode area of around $0.5 \mu \mathrm{m}^{2}$, achieving maximally efficient input coupling would require a 
rather high numerical aperture $(N A \approx 1.25)$ whereas, due to availability, we used an aspheric lens with moderate numerical aperture ( $N A=0.68$, Thorlabs C330TMD-C). With this arrangement, the input coupling loss estimated from throughput measurements amounted to about $75 \%$. The output light from the other waveguide facet was collected via a microscope objective ( $N A=0.74$, Mitutoyo x60) with an estimated output coupling loss of $43 \%$ for the fundamental (as obtained from the measured collimated output from the microscope objective and power measurements with a large area power meter). The fundamental and $\mathrm{SH}$ powers were continuously monitored vs. time. For monitoring the respective spectra, power fractions of about $16 \%$ for the fundamental and 1\% for the second-harmonic power were sent to separate spectrometers.

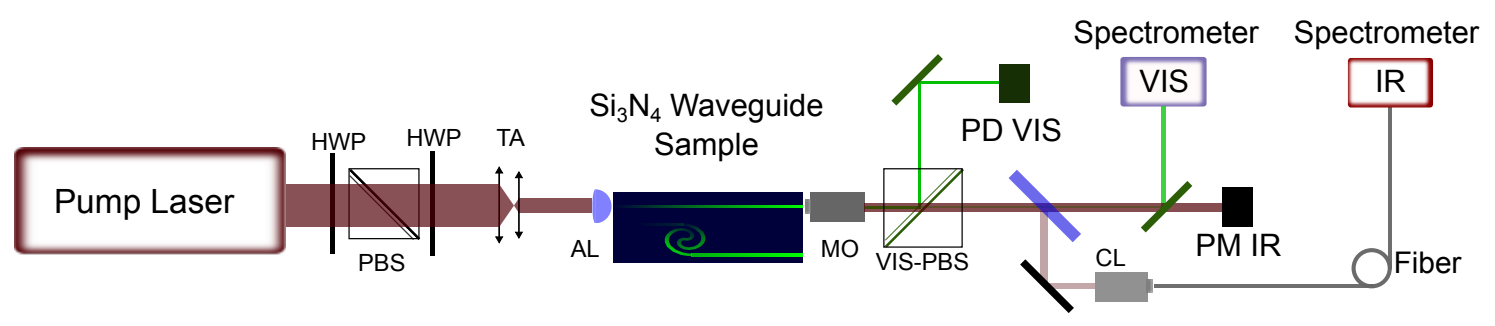

Figure 5.2: Schematic of the experimental setup used for secondharmonic $(\mathrm{SH})$ generation in $\mathrm{Si}_{3} \mathrm{~N}_{4}$ waveguides. The infrared pump laser with a wavelength near $1 \mu \mathrm{m}$ is sent through two half-wave plates (HWP), through a telescope arrangement (TA), and a polarizing beam splitter (PBS) and focused into a waveguide sample with an aspheric lens (AL). Output from the waveguide is collected with a microscope objective (MO), followed by a dichroic mirror (DM) that spectrally separates the fundamental IR from the second-harmonic. A photodiode (PD VIS) behind a polarizing beam splitter (VIS PBS) and a power meter (PM IR) record the SH power and IR radiation vs. time. A small portion of the output ( $1 \%$ of the $\mathrm{SH}$ and $16 \%$ of the IR) is guided into two spectrometers to monitor the visible (VIS) and infrared (IR) spectra, using a collection lens (CL) and a large mode area fiber.

In Figs. 5.3(a-d) several typical examples of time dependent measurements performed during the initialization are shown, i.e., when exposing the waveguides to pump radiation for the first time. The examples comprise two different waveguide widths $(w=0.9 \mu \mathrm{m}$ and $1.2 \mu \mathrm{m})$, and three different waveguide lengths $(L=74,60$ and $22 \mathrm{~mm}$ ). The power of the waveguide-internal pump laser, in these examples using horizontally polarized light, is shown as red traces and was kept constant after turn-on. The traces shown in green display the generated SH vs. time. It can be seen that the SH output does not appear simultaneously with the pump laser turnon. Instead, the $\mathrm{SH}$ builds up with a delay of minutes to hours, after which it rises 
within tens of seconds to minutes until reaching a steady state, with the sooner and steeper growth occurring at higher pump powers.

For comparison with the experimental data we applied a least-square fit using the exponential growth function as given in [32], $f(t)=\frac{a}{1+\left(R t_{0}-1\right) e^{-2 t R}}$, where $a, t_{0}$ and $R$ are fit parameters. In this expression, $a$ is the steady-state value, $t_{0}$ is the delay time and $R$ quantifies the growth rate of the $\mathrm{SH}$ output, i.e., the steepness of the slope at the moment that the output reaches a $1 / e$-fraction of the steady-state value. The figure shows that, for all pump powers, the fit function matches the experimental data very well. For an evaluation of the power dependence, the values for the growth rate retrieved from the fits are displayed as data points in Fig. 5.3(e) vs. the pump power, $P_{p}$. For a comparison, we used a least-square fit of a linear function of the pump power as $R=\left(P_{p}-P_{0}\right) / F$, where $F$ and $P_{0}$ are fit parameters. $P_{0}$ is the pump power where the growth rate becomes zero, indicating the existence of a minimum or threshold pump power required to obtain SHG. Figure 5.3(e) shows that the fit agrees well with the measured growth rates. The threshold pump power retrieved from the fit, $P_{0}=1.22 \mathrm{~mW}$, is indicated in Fig. 5.3(e) with an arrow.

In order to verify that the observed green output is indeed SHG and not radiation from a different process, such as fluorescence from impurity ions, the SH output power was measured vs. the IR pump power. Expected is a quadratic growth with the pump power and that the output shows a well-defined polarization. The experiment was carried out by reducing the pump power towards zero, starting from the value that was used to generate a steady-state $\mathrm{SH}$ output, and also by increasing it from zero back to the maximum available power. In these measurements, performed after the initialization as described above, we observed that the $\mathrm{SH}$ power followed the pump power reduction or increase without any noticeable delay (sampling time $1 \mathrm{~s})$. Also, the output was found linearly polarized, parallel to the polarization of the pump laser. The measured SH output vs. pump power is displayed in Fig. 5.4. A double-logarithmic plot is chosen for easy identification of power laws, where a quadratic dependence shows as a straight line with a slope, $m$, of 2 . The dashed line shown in the figure is a least-square fit with a fixed slope of $m=2$ and with a variable offset as single fit parameter. It can be seen that the generated output power follows very well the expected quadratic dependency of second-harmonic generation. The agreement clearly proves that the investigated waveguides show a second-order nonlinear response.

In order to investigate whether the generated output involves components also of higher-order nonlinear response, specifically, self-phase modulation or four-wave mixing via the $\chi^{(3)}$-nonlinear susceptibility, we recorded the power spectra of the pump laser and the SH output for comparison with each other. If the output is solely dependent on the pump power with a square-law, one expects that the spectral shape of the SH output should match the convolution of the IR spectrum with 

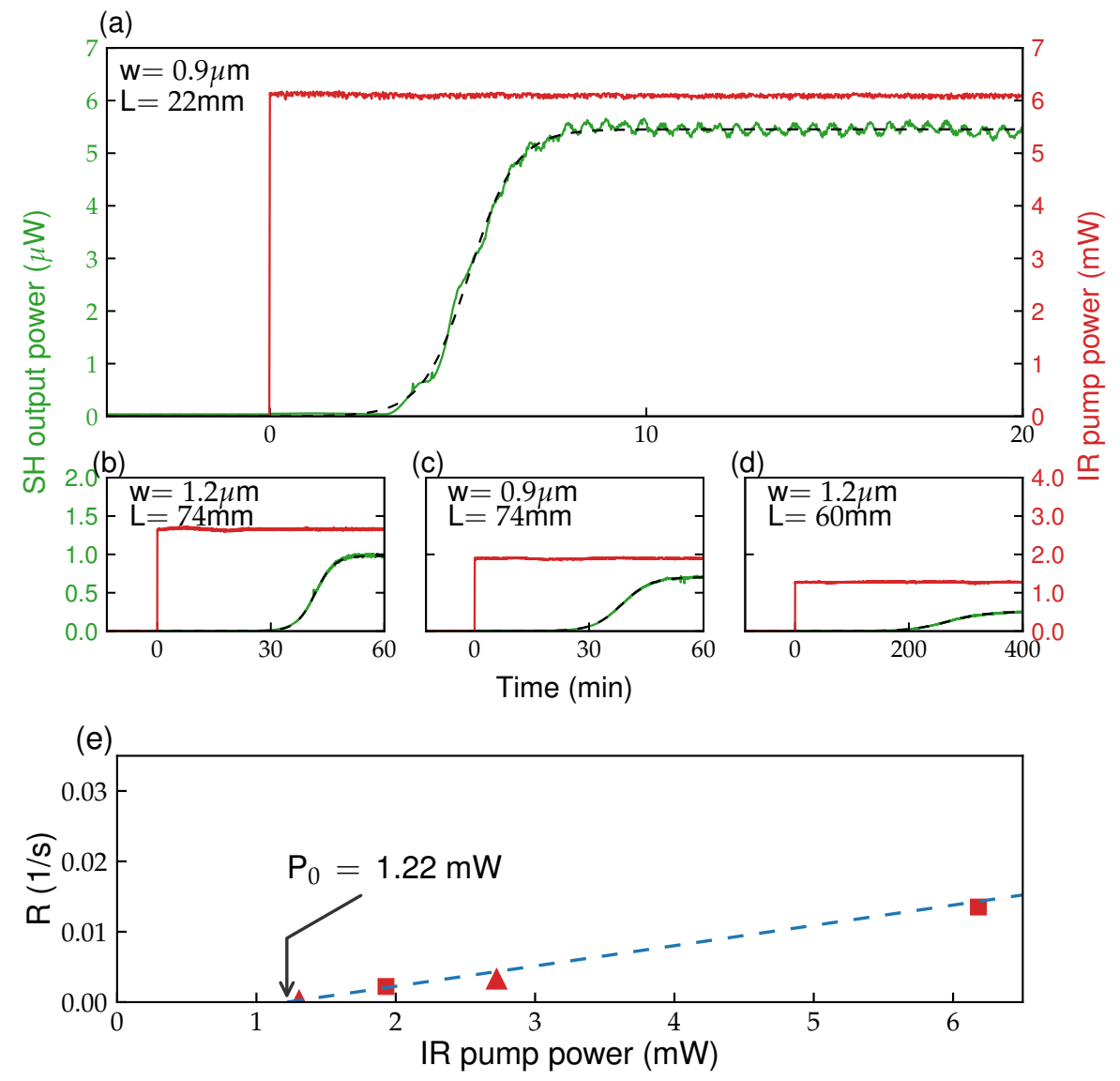

Figure 5.3: (a) to (d). Initial growth of the second-harmonic output power $v s$. time (green traces) in four waveguides of different widths, $w$, and lengths, $L$. The red traces show the average infrared pump power in the waveguide. The dashed curves are least-square fits of the exponential function $f(t)$ (see main text) to the experimental data. (e). Shown is the rate of growth, $R$, where the $\mathrm{SH}$ output has reached a $1 / \mathrm{e}$-fraction of its steady-state value, as retrieved from the fits to the data in (a-d), vs. the waveguide-internal pump power. The triangular and square symbols represent growth rates as obtained with waveguide widths of $w=0.9 \mu \mathrm{m}$ and $1.2 \mu \mathrm{m}$, respectively. The dashed curve is a linear least-square fit to the data. 


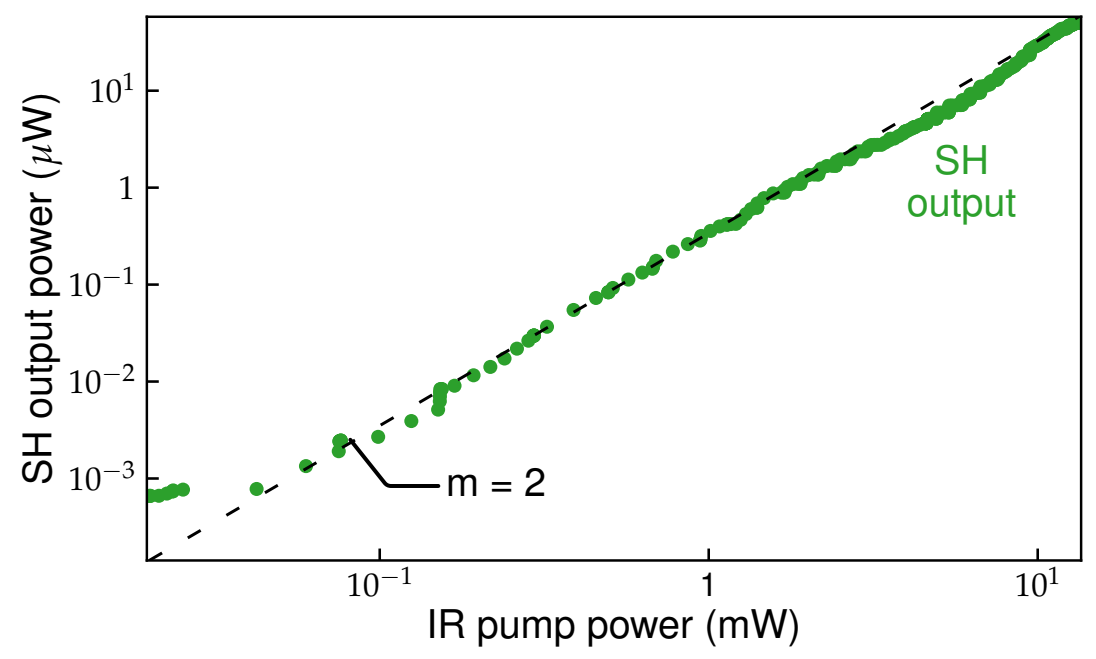

Figure 5.4: Measured second-harmonic ( $\mathrm{SH})$ output as function of input infrared pump power. The used waveguide has a width of $0.9 \mu \mathrm{m}$, a height of $1.0 \mu \mathrm{m}$ and a length of $22 \mathrm{~mm}$. The dashed line is a quadratic fit curve (slope $m=2$ ).

itself (autocorrelation of the IR power spectrum). Figure 5.5 compares an example of a measured and normalized power spectrum of the SH (green trace) with the normalized autocorrelation spectrum of the measured pump spectrum (red trace). It can be seen that the $\mathrm{SH}$ power spectrum fits the autocorrelated pump spectrum well, which excludes noticeable spectral influences of self-phase modulation and four-wave mixing.

A next measurement was performed to attempt clarifying what type of phase-matching mechanism was present that enables generation of the observed SH. Figure 5.1 suggests that modal phase matching should be possible only with a single, specific value for the waveguide width. The experiments, however, showed that SH output is generated regardless of the waveguide width, such that equal phase velocities of transverse modes alone cannot explain the observed phase matching. For instance, SHG is observed with $w=0.9 \mu \mathrm{m}$ as well as with $1.2 \mu \mathrm{m}$ (see Fig. 5.3 and also Fig. 5.7(a)). To gain further information by identifying the spatial mode in which the $\mathrm{SH}$ is generated, we recorded the transverse intensity profile of the visible output in the far-field behind the waveguide, with a microscope objective and a CCD. To provide a large $N A$ for the recording, a microscope objective was placed $(N A=1.4$, 60x, Zeiss) with immersion oil (index of 1.5) slightly closer than the focal distance, in order to maintain a diverging beam for a far-field measurement.

The CCD was positioned about $1 \mathrm{~m}$ beyond the microscope objective. The measured $\mathrm{SH}$ far-field intensity profile, normalized to the maximum intensity, for a waveguide 


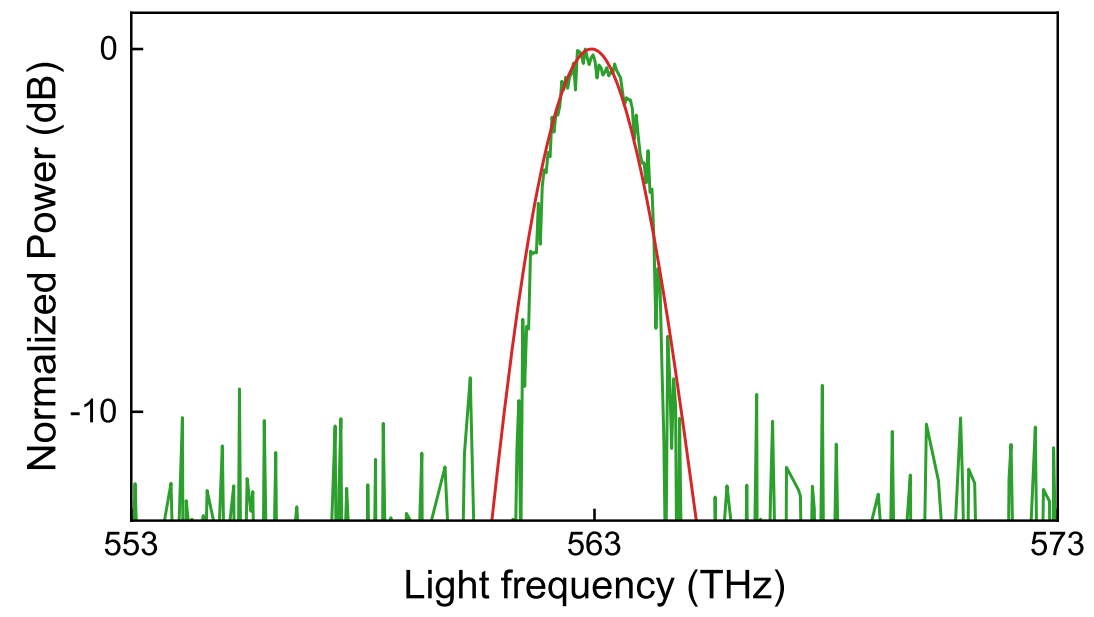

Figure 5.5: Power spectra of the generated visible radiation (green trace) compared with the autocorrelated infrared pump power spectrum (red trace) in a logarithmic plot, with peak values normalized to $0 \mathrm{~dB}$. The $\mathrm{SH}$ was generated in a waveguide with a $0.6 \times 1 \mu \mathrm{m}^{2}$ crosssection and a length of $36 \mathrm{~mm}$, with a waveguide-internal pump power of $4.8 \mathrm{~mW}$.

with a cross-section of $0.7 \times 1 \mu \mathrm{m}^{2}$ and length of $36 \mathrm{~mm}$ is shown in Fig. 5.6(a). Figure 5.6 shows a transverse-moded structure with vertically three main lobes (the lower one and the higher one having the highest intensity, the central one distorted), and with horizontally two weaker side lobes. As the SH intensity profile could only be measured in the far-field, it is not possible to determine the modal decomposition of the $\mathrm{SH}$ to great detail. Further complications are some diffraction by the objective aperture and possible asymmetries introduced by the meniscus of the oil droplet present between waveguide facet and microscope objective ${ }^{1}$. However, the main features in the measured pattern, such as number of lobes and symmetry, allows the identification of the main mode in which the $\mathrm{SH}$ is generated.

To that end, Figs. 5.6(b-d) show the normalized intensity profiles of the waveguide modes $E_{13}, E_{21}$ and $E_{31}$, respectively, for the horizontal polarization. In calculating the transverse profiles of these eigenmodes, the actual waveguide geometry as determined from SEM measurements was used [13]. The waveguide core has rounded edges at the bottom, i.e., at the location of minimum $y$ value in Fig. 5.6, and this asymmetry is responsible for the higher intensity of the lower lobes in the profiles shown in Fig. 5.6. Qualitatively, the presence of a weak central spot with vertically stronger and horizontally weaker side spots, the highest similarity was found with

\footnotetext{
${ }^{1}$ Asymmetries became clearly visible in the experiment when the objective was moved too far from the exit facet of the waveguide
} 

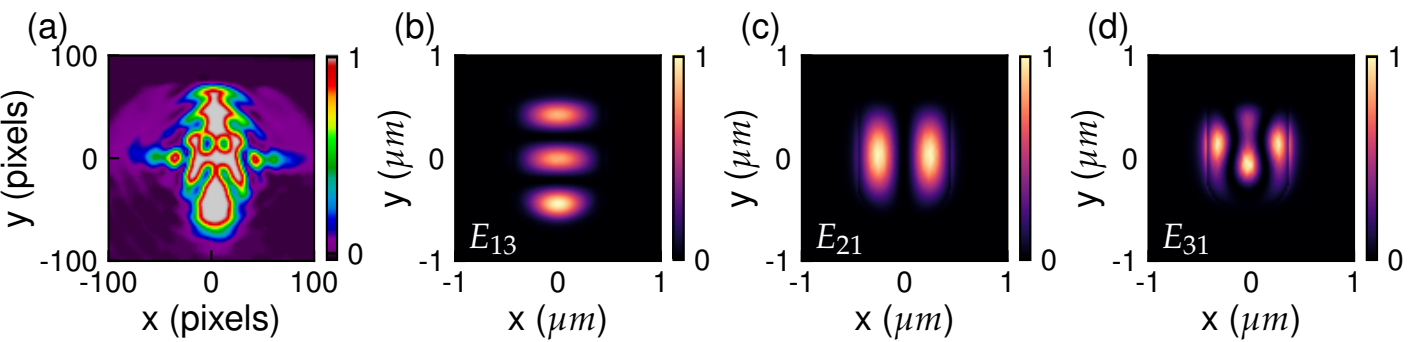

Figure 5.6: Far-field intensity pattern formed by the second-harmonic output beam, normalized to the maximum intensity, recorded with a CCD behind an immersion objective (a) and calculated normalized intensity pattern for the waveguide modes $E_{13}$ (b), $E_{21}$ (c) and $E_{31}$ (d). The waveguide has a width of $0.7 \mu \mathrm{m}$ and a height of $1 \mu \mathrm{m}$. The SH output and waveguide modes are horizontally polarized.

the $\mathrm{E}_{13}$ mode, with some small contributions from either the $\mathrm{E}_{21}$ mode, the $\mathrm{E}_{31}$ mode or both. For reasons discussed below, we also expect that the $\mathrm{SH}$ radiation is dominantly generated in the $\mathrm{E}_{13}$ mode for this waveguide.

Finally, in order to identify the optimum overall conditions for SHG we recorded the dependence of the SHG output vs. the waveguide width, length, and polarization. Figure 5.7(a) shows the measured power for five different waveguide core widths, $0.6,0.7,0.8,0.9,1.1$, and $1.2 \mu \mathrm{m}$ (height of $1 \mu \mathrm{m}$ and length of $36 \mathrm{~mm}$ ), using the same waveguide-internal pump power of $10 \mathrm{~mW}$. Squares represent horizontally polarized light and triangles vertically polarized light. Figure 5.7(b) shows the SH output power as a function of the waveguide length using a fixed waveguide core cross-section $\left(0.9 \times 1 \mu \mathrm{m}^{2}\right)$ and the same waveguide-internal pump power $(10 \mathrm{~mW})$.

A maximum $\mathrm{SH}$ output is found for a waveguide length of $L=36 \mathrm{~mm}$, and a cross-section of $0.9 \times 1 \mu \mathrm{m}^{2}$ for horizontally polarized pumping. Regarding polarization, two effects were observed when changing the input polarization. Initially, i.e., immediately after injecting the other polarization, there was no SH output. Instead, a SH build-up took place with a certain delay and growth rate as described in Fig. 5.3. Thereafter, the SH output followed instantaneously any variation of the pump power. The second observation is that the polarization of the $\mathrm{SH}$ output was always parallel to that of the pump radiation. Independent of polarization effects it was observed that the second-order nonlinear response became erased when illuminating the waveguide with UV radiation (a PR-100, UVP Inc., with a $254 \mathrm{~nm}$ wavelength was used for ten minutes). After storing initialized waveguides in complete darkness at room temperature, typically for at least a week, we did not observe 
(a)

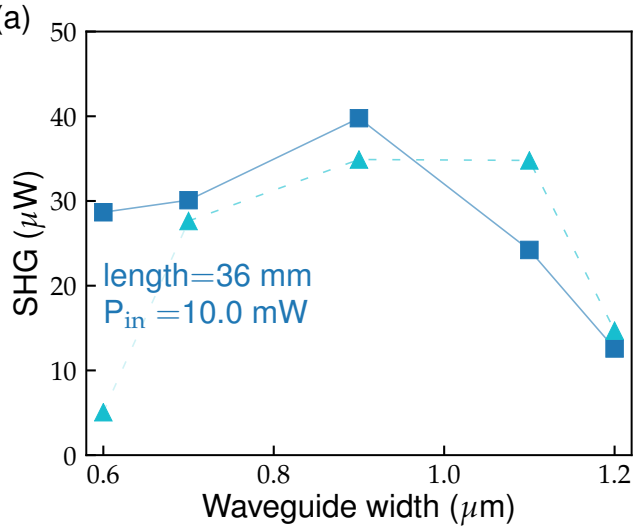

(b)

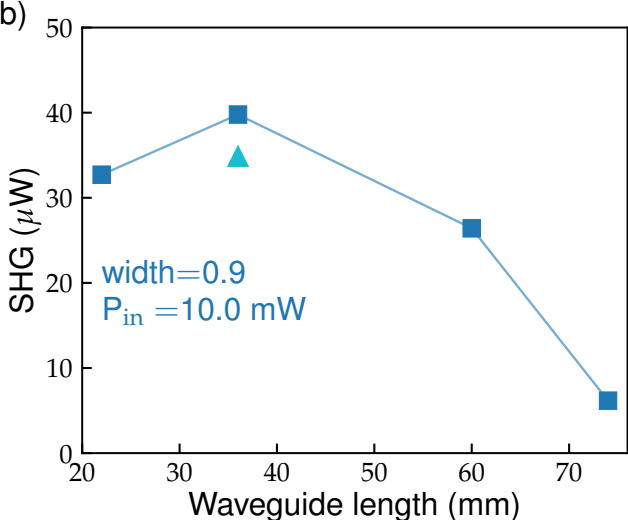

Figure 5.7: (a) Measured second-harmonic (SH) output power for different waveguide widths with a fixed input power of $10 \mathrm{~mW}$, horizontally polarized (squares) and vertically polarized light (triangles) for a waveguide length of $36 \mathrm{~mm}$. (b) Measured second-harmonic power for different waveguide lengths with a fixed input power of $10 \mathrm{~mW}$, for a waveguide with $0.9 \times 1 \mu \mathrm{m}^{2}$ cross-section.

any degradation of the nonlinear response.

\subsection{Discussion}

The most basic expectation that the second-harmonic (SH) output power would follow a quadratic growth with the input pump power, and that the spectrum would have the same spectral bandwidth and shape as the autocorrelated pump spectrum, is confirmed by the experimental observations. However, contrary to what is expected, which is the absence of $\mathrm{SH}$ output, due to fabrication as amorphous materials, we observed second-harmonic generation, though this required an initialization process during which the $\mathrm{SH}$ is time dependent. This time dependency is a growth over time of the $\mathrm{SH}$ power towards a steady-state value, as long as the pump power is above a threshold value $\left(P_{0}\right.$ as indicated in Fig. 5.3(e)). Unexpected is also that SHG was observed in all of the investigated waveguides, independent of their width, which excludes an explanation solely based on modal phase matching. Instead, during the initialization, an additional wave-vector, i.e., a matching spatial periodicity, appears to become available that compensates the remaining wave vector mismatch of modal phase matching.

We address our observation of phase-matched SHG to the so-called coherent photogalvanic effect (CPGE), because of the excellent match of the fit functions to the 
measured temporal growth function and growth-rate vs. power dependence in Figs. 5.3, (a-e)). The temporal fit function was derived by Balakirev et al. [32] for the CPGE in glass. The first observations of the CPGE in guided optics were made with phosphor-doped silica fibers [28, 29, 33-35] and several phenomenological models were proposed [32, 36-39].

In brief, a first part of the CPGE is related to the third-order term in the expansion of Ohm's law, $\mathbf{j}=\sigma \mathbf{E}$, in powers of the electric field [40]. Here, $\mathbf{j}$ is the current density, $\sigma$ is the conductivity and $\mathbf{E}$ a directed (DC) electric field generated by the light fields present in the waveguide. A second, underlying nonlinear effect is third-order optical rectification, which originates from the simultaneous presence of light at the fundamental frequency, $\omega$, and its second harmonic, at $2 \omega$. The $\chi^{(3)}$ nonlinear rectification field induces a photocurrent density that is proportional to $\mathbf{E}(\omega) \mathbf{E}(\omega) \mathbf{E}^{*}(2 \omega)$. This nonlinear DC current density possesses a spatial periodicity set by the wavenumber mismatch between the fundamental and second-harmonic fields $(\Delta k=2 k(\omega)-k(2 \omega))$. The current density leads to a spatial redistribution of electrons between long-lived intra-band trap sites [35, 36, 41], which is the origin for generating an effective second-order nonlinearity. An equivalent picture is the creation of a nonlinear conduction via multi-photon excitation of electrons into the conduction band. Simultaneously, the optical rectification field created via the third-order susceptibility creates a charge relocation between the trap sites with a spatial pattern that provides self-organized quasi-phase matching.

For a somewhat more detailed description, we make the simplification that all optical fields have the same linear polarization, in agreement with our experimental observation. The third-order nonlinear conductivity and susceptibility tensors are assumed to be isotropic and can be represented as scalars as the materials under consideration are amorphous. Accordingly, we treat all fields, current densities and tensors as scalar quantities.

We define the electric field of the fundamental input wave as

$$
E(\omega ; \mathbf{r})=A_{1}(z) E(\omega ; x, y) \cos \left(k(\omega) z+\varphi_{1}\right)
$$

and the second-harmonic field as

$$
E(2 \omega ; \mathbf{r})=A_{2}(z) E(2 \omega ; x, y) \cos \left(k(2 \omega) z+\varphi_{2}\right) .
$$

Here, $z$ is the propagation coordinate, $k(\omega)$ and $k(2 \omega)$ are the wavenumber of fundamental and $\mathrm{SH}$, and $\varphi_{1}$ and $\varphi_{2}$ are phase offsets for the fundamental and $\mathrm{SH}$ fields. $A_{1}(z)$ and $A_{2}(z)$ are the amplitudes of the field distributions along the propagation direction for the fundamental and the SH waves, $E(\omega ; x, y)$ and $E(2 \omega ; x, y)$ represent the transverse field distributions, respectively. 
Electrons, promoted to the conduction band via multi-photon absorption, are driven away from their original locations by a static (DC) electric field, $E_{D C}$, that is generated via the third-order nonlinearity (third-order optical rectification),

$$
P_{D C}^{(3)}(0 ; \mathbf{r})=\varepsilon_{0} \chi^{(3)}(0=\omega+\omega-2 \omega) E(\omega ; \mathbf{r}) E(\omega ; \mathbf{r}) E^{*}(2 \omega ; \mathbf{r}) .
$$

Equation 5.3 shows that the optical rectification field exhibits a spatial modulation set be the wavevector mismatch between the fundamental and second harmonic fields that is contained in the $z$-dependence of $E(\omega ; \mathbf{r})$ and $E(2 \omega ; \mathbf{r})$. Consequently, the associated nonlinear photocurrent density $j_{p h}$ given by $[35,42]$

$$
j_{p h}(\mathbf{r})=C(x, y)\left|A_{1}(z)\right|^{2} A_{2}(z)|E(\omega ; x, y)|^{2} E^{*}(2 \omega ; x, y) \cos (\Delta k z+\Delta \varphi)
$$

exhibits the same spatial modulation. In Eq. 5.4, $C(x, y)$ is the effective photogalvanic coefficient [38, 40,42], $\Delta k=2 k(\omega)-k(2 \omega)$ is the wavenumber mismatch, and $\Delta \varphi$ is a constant. The direction of the current follows the transverse polarization of the inducing light fields. Via this photocurrent and due to the presence of trap-sites, a long-lived charge grating is written into the material which remains present when the optical fields are turned off.

In the presence of light, the charge grating continues to develop, but the development ceases when the transversely orientated space-charge field, $\mathcal{E}_{D C}$, associated with the charge grating balances the optical rectification field,

$$
\varepsilon_{0} \chi^{(1)} \mathcal{E}_{D C}=-\varepsilon_{0} \chi^{(1)} E_{D C}=-P_{D C}^{(3)}(0 ; \mathbf{r}) .
$$

Alternatively expressed, the development of the charge grating will cease when the space-charge field has increased to a level where

$$
\mathcal{E}_{D C}(\mathbf{r})=-j_{p h}(\mathbf{r}) / \sigma_{\text {eff }},
$$

where $\sigma_{\text {eff }}$ is the effective conductivity of the material in the presence of the optical fields. This conductivity is composed of the third-order nonlinear conductivity $\sigma^{(3)}$ and of a background conductivity, also named dark conductivity, which has a low value for the case of amorphous glass [38]. The spatial period of $\mathcal{E}_{D C}(\mathbf{r})$ along $z$ is again given as Eq. 5.4 by the fundamental vs. SH wavenumber mismatch.

Finally, the fundamental light field, $E(\omega, \mathbf{r})$, together with the static field, $\mathcal{E}_{D C}$, generates a second-harmonic polarization via the material's third-order nonlinear response, of which the transverse component is given by

$$
\mathcal{P}^{(3)}(2 \omega ; z)=\varepsilon_{0} \chi^{(3)}(z) \mathcal{E}_{D C}(\mathbf{r}) E(\omega ; \mathbf{r}) E(\omega ; \mathbf{r}) .
$$

This third-order response at the second-harmonic frequency can be seen as an effective second-order polarization based on an effective second-order nonlinearity, as given by [35]

$$
\chi_{\text {eff }}^{(2)}(\mathbf{r})=\chi^{(3)} \mathcal{E}_{D C}(\mathbf{r}) .
$$


In Eq. 5.8, the induced $\mathcal{E}_{D C}$, and thus also $\chi_{\text {eff }}^{(2)}$ assumes the form of a spatial grating along $z$ with the periodicity set by $\Delta k$. The spatial structure of $\chi_{\text {eff }}^{(2)}$ is then similar to a periodically poled second-order nonlinear crystal that provides quasi-phase matching [43], in this case for second-harmonic generation.

\section{Phenomenological description of second harmonic mode selection}

As described above, the CPGE provides quasi-phase matching independent of the wavenumber mismatch. Therefore, a second-harmonic output should be generated independent of the chosen waveguide width which was indeed experimentally observed. However, for the same reason, the generation should then also be possible independent of the specific type of transverse mode in which the second-harmonic field is generated. For instance, SHG should also be generated in the fundamental mode, $\mathrm{E}_{11}(2 \omega)$ by the fundamental IR mode, $\mathrm{E}_{11}(\omega)$. In contrast to this, we have observed the SH output to be generated predominantly in a specific higher-order (transverse) mode shown in Fig. 5.6(a), i.e., in the $\mathrm{E}_{13}$ mode (Fig. 5.6(b)) with only some small contributions from the $\mathrm{E}_{21}$ (Fig. 5.6(c)) or $\mathrm{E}_{31}$ (Fig. 5.6(d)) modes. This rises the question, why the dominant $\mathrm{SH}$ mode is $\mathrm{E}_{13}$. In order to discuss possible reasons, in the following we present a phenomenological description of details in the generation of the spatial charge distribution expected in our case.

To generate an initial photocurrent via an optical rectification field, an initial SH seed wave is required. Initially, the seed wave might posses only a small amplitude. Nevertheless, the nonlinear feedback, enabled by the photocurrent-generated charge grating responsible for the effective second-order nonlinearity that leads again to second-harmonic generation, will eventually lead to an exponential growth starting from the seed wave. An initial SH seed wave can be present, e.g., from surface SHG near the interface between core and cladding [44], near local material anisotropies [36], or by photoionization of defects, i.e., of electrons from dangling bonds $[40,45]$.

Two mechanisms can be responsible for the nonlinear optical generation of a conductivity, required for the coherent photogalvanic effect. The first possibility is excitation of electrons via three-photon absorption from the valance band to the conduction band [41]. This possibility is, however, not likely in our experiments since the bandgap of stoichiometric $\mathrm{Si}_{3} \mathrm{~N}_{4}(4.9 \mathrm{eV})$ is even larger than the energy of four pump photons $(4.6 \mathrm{eV})$. The second mechanism is via dangling bonds in stoichiometric silicon nitride. In LPCVD $\mathrm{Si}_{3} \mathrm{~N}_{4}$ two main types of dangling bonds are possible, silicon (K-centers, having an energy of $\sim 2.6 \mathrm{eV}$ above the upper edge of the valence band) and nitrogen (N-centers, having an energy of $\sim 0.3 \mathrm{eV}$ above the upper edge of the valence band) [46]. These dangling bonds allow photoexcitation of electrons into the conduction band. As the dangling bond defects are most stable in their charged 


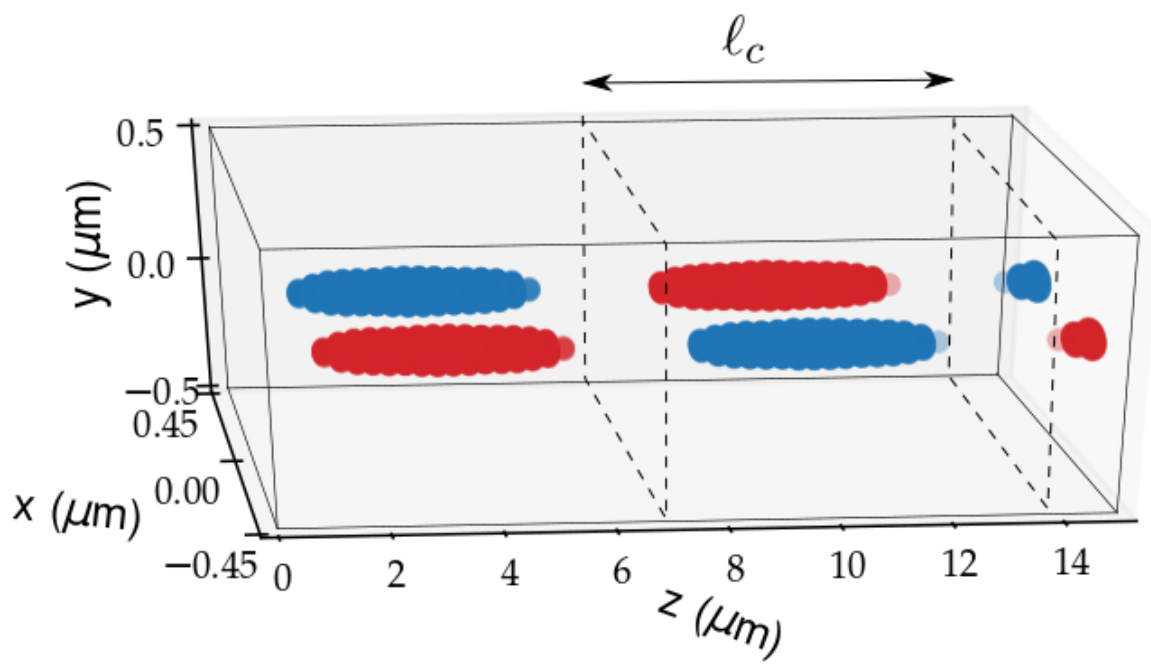

Figure 5.8: Normalized charge distribution in the waveguide core induced by the coherent photogalvanic effect. The generating optical fields are assumed to be the fundamental mode $E_{11}(\omega ; \mathbf{r})$ for the IR field and the $\mathrm{E}_{13}(2 \omega ; \mathbf{r})$ mode for the $\mathrm{SH}$ field, both polarized along the $x$-direction. For clarity only charge values with $|\rho|$ above $80 \%$ of the maximum value are plotted, where red and blue represents positive and negative charge, respectively. The sign of the effective $\chi^{(2)}$ is then given from blue to red. The horizontal arrow indicates the coherence length for SHG, $\ell_{c}=\frac{\pi}{\Delta k}$, in absence of quasi-phase matching.

state [47] they also act as trap sites and provide the mechanism to create long-lived charge gratings in the core of the waveguide.

The establishment of the charge grating may be described and understood as follows. The time dependency of the $\mathrm{SH}$ output is dominated by the excitation of charges by the pulsed laser (ps timescale), while the recombination time of charges to a vacancy is rather long ( $\sim 0.1 \mathrm{~s})$ [48]. Due to the high repetition rate of the laser pulses present over a significant illumination time, the excited electrons can be pushed away by the optical rectification field from volume elements where the product of the light fields, $E^{*}(2 \omega ; \mathbf{r}) \cdot E(\omega ; \mathbf{r})^{2}$, is large, towards trap sites in the direction given by the rectification field. If the optical rectification field is not strong enough, recombination will be dominant and no charge grating is written, i.e., the average IR laser power should be above a threshold, which is in agreement with our experimental observation ( $c f$. Fig. 5.3(e)).

To illustrate the shape of charge distribution written in the core of the waveguide, we have calculated the product $E(\omega ; \mathbf{r})^{2} E^{*}(2 \omega ; \mathbf{r}) \propto \mathcal{E}$ and used Poisson's equation, simplified to $\rho(x) \propto d \mathcal{E} / d x$, where $\rho$ is the charge density, to determine from the transverse field the according charge density. Figure 5.8 shows the example of a 
charge distribution as generated by the pump radiation in the $\mathrm{E}_{11}$ mode and by $\mathrm{SH}$ in the $\mathrm{E}_{13}$ mode, both having a horizontal polarization. For better clarity of presentation, only charge levels with $|\rho|$ in the range of $80 \%-100 \%$ of the maximum charge are shown. Positive and negative charge are indicated by red and blue, respectively. Figure 5.8 shows that the overlapping pump and $\mathrm{SH}$ fields generate a charge grating where the longitudinal periodicity defines a grating period, $\Lambda=2 \ell_{c}=\frac{2 \pi}{\Delta k}$, where $\ell_{c}$ is the coherence length for second-harmonic generation in absence of quasi-phase matching. This grating period is what is required for quasi-phase matched (QPM) generation of the second harmonic field.

At this point it can be discussed, why not all of the allowed spatial modes are equally likely to generate a SH output. We suggest that the answer lies in the very different charge patterns that correspond to the different pump and $\mathrm{SH}$ mode combinations. As can be seen in Fig. 5.8 the longitudinal grating period, $\Lambda$, is set by the wavenumber mismatch between the mode of the IR pump, here taken to be the fundamental $\mathrm{E}_{11}$ mode, and the mode of the $\mathrm{SH}$ field. A smaller phase mismatch, $\Delta k$, and consequently a larger grating period, $\Lambda$, results in a stronger growth with distance for the $\mathrm{SH}$ in the waveguide [44]. Initially, the $\mathrm{SH}$ field might be emitted in various modes. But then, during the initiation phase of exponential growth, the various resulting charge gratings will compete with each other to establish the charge grating in the waveguide. The mode with the largest growth, i.e., with the smallest phase mismatch, will become the dominant mode due to the nonlinear feedback process described above. To illustrate this also quantitatively, we have, for convenience, in Fig. 5.9 replotted Fig. 2.6, however for the waveguide height and shape as present in the experiment. Figure 5.9 depicts the QPM period, $\Lambda$, as a function of the width of the waveguide core for various modes with horizontal polarization.

Figure 5.9 shows that, for a waveguide width of $w=0.7 \mu \mathrm{m}$ i.e., for the waveguide used to record the SH beam profile in Fig. 5.6(a), the required QPM period, $\Lambda$, is rather short for most of the $\mathrm{SH}$ modes, and consequently these modes will only experience a moderate growth. Only certain modes, here $E_{13}$, can be quasi-phase matched with a long QPM period and will experience a far larger growth, i.e, will become the dominant mode. This is in agreement with the measured $\mathrm{SH}$ beam profile (see Fig. 5.6(a).

As a related conclusion, from Fig. 5.9, it appears more likely that the observed $\mathrm{SH}$ output is a $E_{13}-E_{21}$ superposition (with dominating $E_{13}$ ) than having a $E_{31}$ contribution. The $\mathrm{E}_{31}$ contribution, that may be present in the observed $\mathrm{SH}$ intensity distribution, requires an even shorter QPM period at $w=0.7 \mu \mathrm{m}$ and is expected to have a smaller growth. Based on these phase-matching arguments, Fig. 5.9 would imply that $\mathrm{SH}$ generated in the $\mathrm{E}_{22}$ mode grows faster than that generated in the $\mathrm{E}_{21}$ mode. However, this mode is not expected to grow due to the zero on-axis intensity of the $E_{22}$ mode, which creates a poor modal overlap of this mode with the $E_{11}$ mode of 


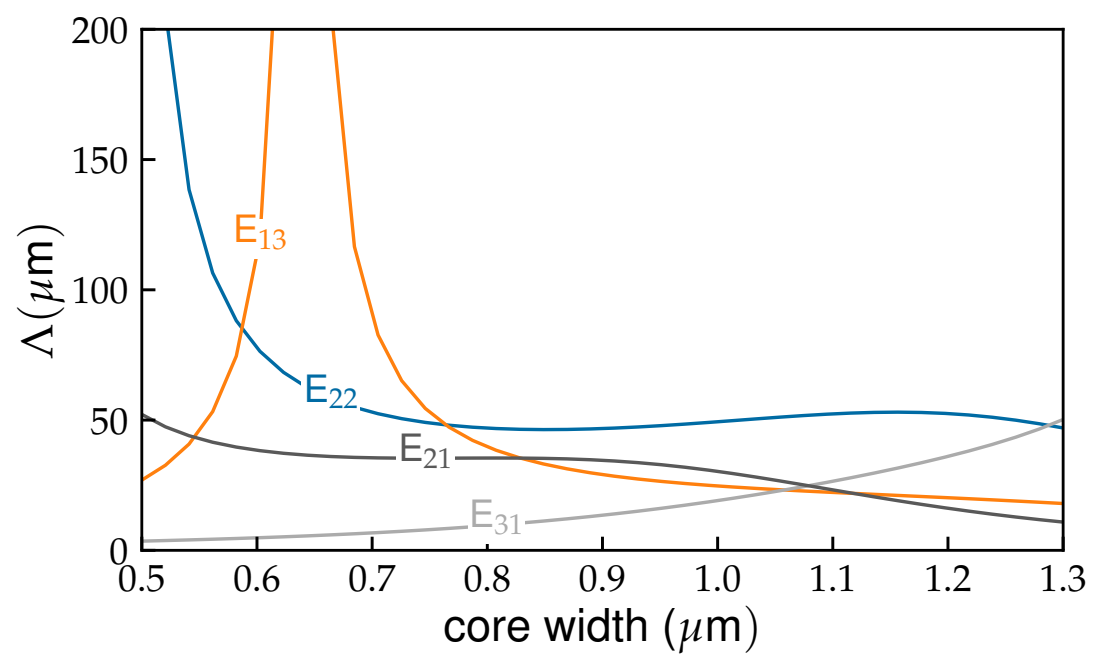

Figure 5.9: QPM period required for SHG in the various different modes shown in Fig. 5.1 with the infrared pump power in the $\mathrm{E}_{11}$ mode. All modes are horizontally polarized. As can be seen, $\mathrm{E}_{13}$ has the largest period for the range of waveguide widths discussed in Fig. 5.1.

the IR pump.

In order to quantify the effective nonlinear susceptibility than can be provided by the CPGE in $\mathrm{Si}_{3} \mathrm{~N}_{4}$ waveguides, we evaluate the maximum power conversion efficiency, $\eta$, which was obtained for a width of $w=0.9 \mu \mathrm{m}$ (core cross-section $0.9 \times$ $\left.1 \mu \mathrm{m}^{2}\right)$. The conversion efficiency is defined as

$$
\eta=P(2 \omega) / P_{0}(\omega)=\kappa^{2} P_{0}(\omega) L^{2},
$$

where $P(2 \omega)$ and $P_{0}(\omega)$ are the generated SH power and the input IR power, respectively, $\kappa$ is the coefficient for nonlinear coupling between the fundamental and $\mathrm{SH}$ waves, and $L$ is the length of the waveguide. Equation 5.9 can be used to determine $\kappa$ from measured powers and the waveguide length. Making use of Eq. 2.20, and assuming perfect quasi-phase matching and no pump depletion, the obtained value for $\kappa$ can be used to determine the effective second-order nonlinear susceptibility, $\chi_{\mathrm{eff}}^{(2)}$ as

$$
\chi_{\mathrm{eff}}^{(2)}=2 \sqrt{\left(\frac{\kappa}{\varepsilon_{0}}\right)^{2} \frac{2\left(n(\omega)_{\mathrm{eff}}\right)^{2} n(2 \omega)_{\mathrm{eff}}}{(2 \omega)^{2}}\left(\frac{\mu_{0}}{\varepsilon_{0}}\right)^{-3 / 2} S_{\mathrm{eff}}},
$$

where $n_{\text {eff }}(\omega)$ and $n_{\text {eff }}(2 \omega)$ are the refractive indices for the IR and SH waves, while $S_{\text {eff }}$ as given by Eq. 2.21, is the effective cross-section of the modal overlap of the IR and the $\mathrm{SH}$ fields. In our case, the horizontally polarized pump radiation is injected in the $\mathrm{E}_{11}$ mode and the $\mathrm{SH}$ is dominantly generated in the $\mathrm{E}_{13}$ mode with the same 


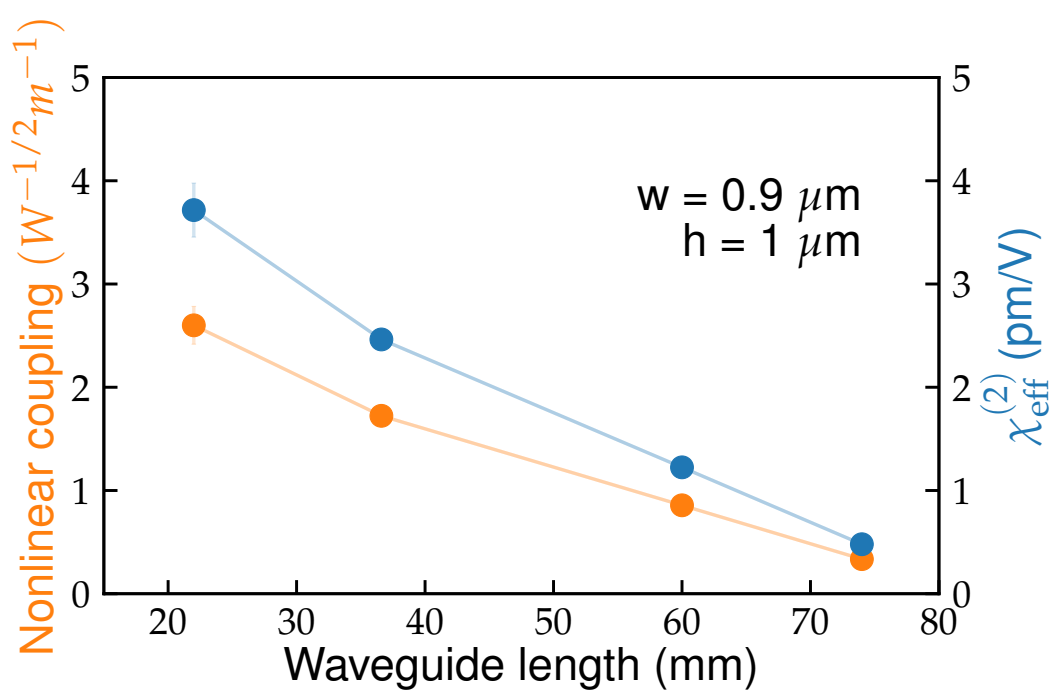

Figure 5.10: Nonlinear coupling (orange markers) and effective $\chi^{(2)}$ (blue markers) as a function of the length of the waveguide. The core of the waveguide has a cross-section of $0.9 \times 1 \mu \mathrm{m}^{2}$. The shown error bar $(0.2 \mathrm{pm} / \mathrm{V}$ half-width) seen at the highest data points represents the statistical uncertainty of the fit in Fig. 5.4 and the experimental uncertainty in measuring the output coupling efficiency at the waveguide exit facet.

polarization. Figure 5.10 shows the experimentally obtained nonlinear coupling, $\kappa$, as a function of the length of the waveguide. Also shown is the corresponding effective second-order susceptibility as calculated using Eq. 5.10, assuming a pure $\mathrm{E}_{13}$ mode for the $\mathrm{SH}$ output. Figure 5.10 shows that the maximum effective value for $\chi^{(2)}$ obtained in this way is about $3.7 \pm 0.2 \mathrm{pm} / \mathrm{V}$. We note that taking into account a small $\mathrm{SH}$ contribution in the $\mathrm{E}_{21}$ mode would lead to a larger effective susceptibility.

\subsubsection{Summary and conclusions}

For the first time, we have observed second-harmonic generation in stoichiometric $\mathrm{Si}_{3} \mathrm{~N}_{4}$ waveguides grown via LPCVD. Second-harmonic output was generated independent of the width of the waveguide core cross-section and required a build-up, a delay in the order of several minutes up to hours and an exponential growth to a steady state value, depending on the IR pump power and waveguide core crosssection. These findings are consistent with the coherent photogalvanic effect being responsible for the building up of a second-order nonlinearity and quasi-phase matching. With the available pump laser pulses, the investigated $\mathrm{Si}_{3} \mathrm{~N}_{4}$ waveguides provide a maximum conversion efficiency of $0.4 \%$ for an input power of $13 \mathrm{~mW}$ 
(peak power $105 \mathrm{~W}$, pulse energy of $0.65 \mathrm{~nJ}$ ). This is the highest second-order conversion efficiency achieved to our knowledge in an integrated SiN platform. The measured conversion efficiency corresponds to a high effective second-order susceptibility, $\chi_{\text {eff }}^{(2)}=3.7 \pm 0.2 \mathrm{pm} / \mathrm{V}$, which is smaller than that from highly Si-rich SiN thin films $\left(d_{\mathrm{eff}}=5.9 \mathrm{pm} / \mathrm{V}\right.$, corresponding to a $\left.\chi_{\text {eff }}^{(2)}=11.8 \mathrm{pm} / \mathrm{V}\right)[26]$ as concluded from X-ray photoelectron spectroscopy (XPS), and slightly larger than that achieved with periodic gratings in thin SiN films $\left(\chi^{(2)}=2.5 \mathrm{pm} / \mathrm{V}\right)$ [49] or in SiN ring resonators $\left(\chi^{(2)}<0.04 \mathrm{pm} / \mathrm{V}\right)$ [25], both calculated from frequency conversion measurements.

Numerous applications are already based on $\chi^{(1)}$-gratings in waveguides such as reconfigurable Bragg filters [6], optical switching [30, 50], mode conversion [51] or optical storage [10]. The presence of an effective $\chi^{(2)}$-grating in amorphous $\mathrm{Si}_{3} \mathrm{~N}_{4}$ waveguides may lead to the development of further functionalities such as parametric down-conversion [52], all optical signal processing [50], and possibly also self-referencing of frequency combs that exploits the simultaneous presence of $\chi^{(2)}$ and $\chi^{(3)}$ in low-loss $\mathrm{Si}_{3} \mathrm{~N}_{4}$ waveguides [53-55].

\section{References}

1. Pavesi, L. Silicon Photonics (Springer Science \& Business Media, 2004).

2. Tekin, T., Pleros, N., Pitwon, R. \& Hakansson, A. Optical Interconnects for Data Centers (Woodhead Publishing, 2016).

3. Hunsperger, R. Integrated Optics: Theory and Technology (Springer, 2013).

4. Smit, M. et al. An introduction to InP-based generic integration technology. Semicond. Sci. Technol. 29, 083001 (2014).

5. Bauters, J. F., Heck, M. J. R., John, D., Dai, D., Tien, M.-C., Barton, J. S., Leinse, A., Heideman, R. G., Blumenthal, D. J. \& Bowers, J. E. Ultra-low-loss high-aspect-ratio $\mathrm{Si}_{3} \mathrm{~N}_{4}$ waveguides. Opt. Express 19, 3163-3174 (2011).

6. Marpaung, D., Roeloffzen, C., Heideman, R., Leinse, A., Sales, S. \& Capmany, J. Integrated microwave photonics. Laser Photon. Rev. 7, 506-538 (2013).

7. Vlasov, Y., Green, W. M. J. \& Xia, F. High-throughput silicon nanophotonic wavelengthinsensitive switch for on-chip optical networks. Nat. Photon. 2, 242-246 (2008).

8. Monat, C., Domachuk, P. \& Eggleton, B. J. Integrated optofluidics: A new river of light. Nat. Photon. 1, 106-114 (2007).

9. Yang, W., Conkey, D. B., Wu, B., Yin, D., Hawkins, A. R. \& Schmidt, H. Atomic spectroscopy on a chip. Nat. Photon. 1, 331-335 (2007).

10. Calafiore, G., Koshelev, A., Dhuey, S., Goltsov, A., Sasorov, P., Babin, S., Yankov, V., Cabrini, S. \& Peroz, C. Holographic planar lightwave circuit for on-chip spectroscopy. Light Sci. Appl. 3, e203 (2014).

11. Spencer, D. T., Bauters, J. F., Heck, M. J. R. \& Bowers, J. E. Integrated waveguide coupled $\mathrm{Si}_{3} \mathrm{~N}_{4}$ resonators in the ultrahigh-Q regime. Optica 1, 153-157 (2014). 
12. Luke, K., Okawachi, Y., Lamont, M. R. E., Gaeta, A. L. \& Lipson, M. Broadband midinfrared frequency comb generation in a $\mathrm{Si}_{3} \mathrm{~N}_{4}$ microresonator. Opt. Lett. 40, 4823-4826 (2015).

13. Epping, J. P., Hoekman, M., Mateman, R., Leinse, A., Heideman, R. G., van Rees, A., van der Slot, P. J. M., Lee, C. J. \& Boller, K.-J. High confinement, high yield $\mathrm{Si}_{3} \mathrm{~N}_{4}$ waveguides for nonlinear optical applications. Opt. Express 23, 642-648 (2015).

14. Wörhoff, K., Heideman, R. G., Leinse, A. \& Hoekman, M. TriPleX: a versatile dielectric photonic platform. Adv. Opt. Technol. 4, 189-207 (2015).

15. Fan, Y., Oldenbeuving, R. M., Klein Edwin J.and Lee, C. J., Song, H., Khan, M. R. H., Offerhaus, H. L., van der Slot, P. J. M. \& Boller, K.-J. A hybrid semiconductor-glass waveguide laser. Proc.SPIE 9135 (2014).

16. Fan, Y., Epping, J. P., Oldenbeuving, R. M., Roeloffzen, C. G. H., Hoekman, M., Dekker, R., Heideman, R. G., van der Slot, P. J. M. \& Boller, K.-J. Optically Integrated InP-Si ${ }_{3} \mathrm{~N}_{4}$ Hybrid Laser. IEEE Photonc J. 8, 1-11 (2016).

17. Roeloffzen, C. G. H., Zhuang, L., Taddei, C., Leinse, A., Heideman, R. G., van Dijk, P. W. L., Oldenbeuving, R. M., Marpaung, D. A. I., Burla, M. \& Boller, K.-J. Silicon nitride microwave photonic circuits. Opt. Express 21, 22937 (2013).

18. Epping, J. P. et al. On-chip visible-to-infrared supercontinuum generation with more than $495 \mathrm{THz}$ spectral bandwidth. Opt. Express 23, 19596-19604 (2015).

19. Porcel, M. A. G. et al. Two-octave spanning supercontinuum generation in stoichiometric silicon nitride waveguides pumped at telecom wavelengths. Opt. Express 25, 15421554 (2017).

20. Epping, J. P., Kues, M., van der Slot, P. J. M., Lee, C. J., Fallnich, C. \& Boller, K.-J. Integrated CARS source based on seeded four-wave mixing in silicon nitride. Opt. Express 21, 32123-32129 (2013).

21. Pfeifle, J. et al. Coherent terabit communications with microresonator Kerr frequency combs. Nat. Photon. 8, 375-380 (2014).

22. Hellwig, T., Epping, J. P., Schnack, M., Boller, K.-J. \& Fallnich, C. Ultrafast, low-power, all-optical switching via birefringent phase-matched transverse mode conversion in integrated waveguides. Opt. Express 23, 19189-19201 (2015).

23. Xiong, C. et al. Compact and reconfigurable silicon nitride time-bin entanglement circuit. Optica 2, 724 (2015).

24. Ning, T., Pietarinen, H., Hyvärinen, O., Simonen, J., Genty, G. \& Kauranen, M. Strong second-harmonic generation in silicon nitride films. Appl. Phys. Lett. 100, 161902 (2012).

25. Levy, J. S. \& Lipson, M. Harmonic generation in silicon nitride ring resonators. Opt. Express 17, 11366-11370 (2011).

26. Kitao, A., Imakita, K., Kawamura, I. \& Fujii, M. An investigation into second harmonic generation by Si-rich $\mathrm{SiN}$ thin films deposited by RF sputtering over a wide range of Si concentrations. J. Phys. D: Appl. Phys. 47, 215101 (2014).

27. Torres-Company, V. \& Weiner, A. M. Optical frequency comb technology for ultrabroadband radio-frequency photonics. Laser Photon. Rev. 8, 368-393 (2014).

28. Österberg, U. \& Margulis, W. Dye laser pumped by Nd: YAG laser pulses frequency doubled in a glass optical fiber. Opt. Lett. 11, 516-518 (1986).

29. Margulis, W. \& Österberg, U. Second-harmonic generation in optical glass fibers. J. Opt. Soc. Am. B 5, 312-316 (1988). 
30. Suhara, T. \& Fujimura, M. Waveguide Nonlinear-Optic Devices red. by Kamiya, T., Monemar, B., Venghaus, H. \& Yamamoto, Y. (Springer Berlin Heidelberg, Berlin, Heidelberg, 2003).

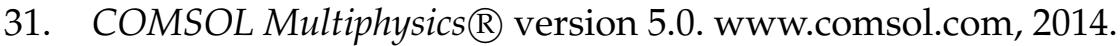

32. Balakirev, M. K., Vostrikova, L. I., Smirnov, V. A. \& Entin, M. V. Relaxation of the optical density of glass modulated with bichromatic radiation. J. Exp. Theor. Phys. Lett. 63, 176181 (1996).

33. Stolen, R. H. \& Tom, H. W. K. Self-organized phase-matched harmonic generation in optical fibers. Opt. Lett. 12, 585-587 (1987).

34. Mizrahi, V., Österberg, U., Sipe, J. E. \& Stegeman, G. I. Test of a model of efficient second-harmonic generation in glass optical fibers. Opt. Lett. 13, 279 (1988).

35. Dianov, E. M. \& Starodubov, D. S. Photoinduced generation of the second harmonic in centrosymmetric media. Quantum Electron. 25, 395-407 (1995).

36. Anderson, D. Z., Mizrahi, V. \& Sipe, J. E. Model for second-harmonic generation in glass optical fibers based on asymmetric photoelectron emission from defect sites. Opt. Lett. 16, 796-798 (1991).

37. Sokolov, V. O. \& Sulimov, V. B. Formation of electrostatic field gratings in glass by optical and acoustical waves in Optics Quebec (International Society for Optics and Photonics, 1993), 213-222.

38. Sokolov, V. O. \& Sulimov, V. B. On the phenomenological theory of the coherent photogalvanic effect in glass. Phys. Status Solidi B 187, 177-187 (1995).

39. Chmela, P. \& Petráek, J. First demonstration of $\chi^{(2)}$ grating encoding function in optical fibers. Opt. Commun. 156, 374-383 (1998).

40. Sokolov, V. O. \& Sulimov, V. B. Theory of third-order nonlinear photoconductivity and third-order photogalvanic effect in glass. Phys. Status Solidi B 187, 189-204 (1995).

41. Balakirev, M. K., Kityk, I. V., Smirnov, V. A., Vostrikova, L. I. \& Ebothe, J. Anisotropy of the optical poling of glass. Phys. Rev. A 67, 023806 (2003).

42. Balakirev, M. K. \& Smirnov, V. A. Kinetics of photoinduced optical anisotropy in oxide glass. J. Exp. Theor. Phys. Lett. 77, 217-220 (2003).

43. Fejer, M. M., Magel, G. A., Jundt, D. H. \& Byer, R. L. Quasi-phase-matched second harmonic generation: tuning and tolerances. IEEE J. Quant. Electron. 28, 2631-2654 (1992).

44. Boyd, R. W. Nonlinear Optics (Academic Press, 2013).

45. Warren, W. L., Robertson, J. \& Kanicki, J. Si and N dangling bond creation in silicon nitride thin films. Appl. Phys. Lett. 63, 2685-2687 (1993).

46. Krick, D. T., Lenahan, P. M. \& Kanicki, J. Electrically active point defects in amorphous silicon nitride: An illumination and charge injection study. J. Appl. Phys. 64, 3558-3563 (1988).

47. Robertson, J., Warren, W. L. \& Kanicki, J. Nature of the Si and N dangling bonds in silicon nitride. Journal of Non-Crystalline Solids. Amorphous Insulating Thin Films II 187, 297-300 (1995).

48. Dianov, E. M., Kazanskii, P. G. \& Starodubov, D. S. Transient gratings of a quadratic nonlinear susceptibility due to the coherent photogalvanic effect in semiconductormicrocrystallite-doped glasses. Quantum Electron. 24, 632-635 (1994). 
49. Ning, T., Pietarinen, H., Hyvärinen, O., Kumar, R., Kaplas, T., Kauranen, M. \& Genty, G. Efficient second-harmonic generation in silicon nitride resonant waveguide gratings. Opt. Lett. 37, 4269-4271 (2012).

50. Stegeman, G. I., Hagan, D. J. \& Torner, L. $\chi^{(2)}$ cascading phenomena and their applications to all-optical signal processing, mode-locking, pulse compression and solitons. Opt. Quantum Electron. 28, 1691-1740 (1996).

51. Chen, G. F. R., Wang, T., Donnelly, C. \& Tan, D. T. H. Second and third order dispersion generation using nonlinearly chirped silicon waveguide gratings. Opt. Express 21, 29223 (2013).

52. Čáp, J. \& Chmela, P. Self-organized parametric down conversion and photoinduced reflectivity in optical fibers. Opt. Quantum Electron. 35, 1079-1090 (2003).

53. Telle, H. R., Steinmeyer, G., Dunlop, A. E., Stenger, J., Sutter, D. H. \& Keller, U. Carrierenvelope offset phase control: A novel concept for absolute optical frequency measurement and ultrashort pulse generation. Appl. Phys. B 69, 327-332 (1999).

54. Jones, D. J., Diddams, S. A., Ranka, J. K., Stentz, A., Windeler, R. S., Hall, J. L. \& Cundiff, S. T. Carrier-Envelope Phase Control of Femtosecond Mode-Locked Lasers and Direct Optical Frequency Synthesis. Science 288, 635-639 (2000).

55. Mayer, A. S., Klenner, A., Johnson, A. R., Luke, K., Lamont, M. R. E., Okawachi, Y., Lipson, M., Gaeta, A. L. \& Keller, U. Frequency comb offset detection using supercontinuum generation in silicon nitride waveguides. Opt. Express 23, 15440 (2015). 


\section{Chapter 6}

\section{Conclusion and outlook}

In this thesis we have explored new approaches to exploit nonlinear properties of $\mathrm{Si}_{3} \mathrm{~N}_{4} / \mathrm{SiO}_{2}$ waveguides. We have investigated nonlinear optical processes based on the nonlinear susceptibility and have shown that these processes can be used to extend the control over light propagating through stoichiometric silicon nitride waveguides. In particular, we have experimentally demonstrated that the spectrum of ultra-short pulses ( 100 femtosecond) with a center wavelength of $1560 \mathrm{~nm}$ can be broadened to $454 \mathrm{THz}$ via supercontinuum generation, which relies on the thirdorder nonlinear susceptibility (Chapter 3). The bandwidth is close to the maximum bandwidth ever reported for supercontinuum generation on a chip [1]. To explore the options for acousto-optic interactions, we used theoretical modeling. The results demonstrate that surface acoustic waves can be used to implement highfrequency phase modulation in these waveguides, which is sufficiently strong to realize compact amplitude modulators based on a balanced Mach-Zehnder interferometer (Chapter 4). Finally, we have experimentally demonstrated that short optical pulses ( $\sim 10$ picoseconds) having a wavelength of $1064 \mathrm{~nm}$ can be frequency doubled via second-harmonic generation, which relies on the creation of a long-lived effective second-order susceptibility via the coherent photogalvanic effect (Chapter 5).

In Chapter 3, we demonstrated extremely wide-bandwidth supercontinuum generation in stoichiometric $\mathrm{Si}_{3} \mathrm{~N}_{4}$ waveguides, using a standard erbium-doped fiber pump laser that provided ultrashort pulses in the telecommunications wavelength range at $1560 \mathrm{~nm}$. In a 6-mm long waveguide with a core area of $1.0 \times 0.9 \mu \mathrm{m}^{2}$ and pumped by a 120-fs long optical pulse with a pulse energy of $4.7 \mathrm{~nJ}$ incident on the aspheric lens, used to coupled light into the waveguide, the supercontinuum radiation showed a spectral bandwidth of more than $454 \mathrm{THz}$, spanning at least 2.2 octaves, from the visible (at $526 \mathrm{~nm}$ ) to the mid-infrared (beyond $2584 \mathrm{~nm}$ ).

The spectral shapes of the generated supercontinua are in very good agreement with theoretical modeling via numerical integration of the Generalized Nonlinear 
Schrödinger equation (GNLSE). Based on this agreement, a comparison of the required waveguide-internal pump pulse energy in the simulation and the measurements was performed. Comparing the best estimate of the experimental optical pulse energy coupled into the waveguide with the theoretically needed value to match the measured supercontinuum spectrum reveals that the model predicts a factor of 3.6 lower required pulse energy. From this we conclude that the measured and model results are in agreement when the intensity dependent index, $n_{2}$, takes the value of $0.67 \times 10^{-15} \mathrm{~cm}^{2} \mathrm{~W}^{-1}$, which is lower than the value reported in literature $[2,3]$. The different values reported for $n_{2}$ are most likely due to the different manufacturing techniques used, however this requires further study.

Control over the supercontinuum, i.e, bandwidth and central wavelength, was obtained through dispersion engineering via the cross-section of the waveguide core, which ideally puts the pump wavelength in the anomalous dispersion region close to the zero-dispersion wavelength. For example, using ultra-short pump pulses with a $1064 \mathrm{~nm}$ center wavelength, the widest supercontinuum spectrum on a chip, with a bandwidth of almost $500 \mathrm{THz}$, has been observed using similar waveguides [1]. Dispersion engineering the waveguides to use shorter (e.g., Ti:sapphire laser) and longer (e.g., Tm-doped fiber laser) pump wavelengths as well as by engineering the drive pulses' temporal chirp would further differentiate the properties of the supercontinuum generated. Such supercontinua are highly attractive as on-chip tunable light sources for label-free microscopy and imaging in bio- and lifesciences $[4,5]$ and spectroscopy.

In Chapter 4 we theoretically investigated the use of Rayleigh-type surface acoustic waves (SAWs) to modulate the effective refractive index of an optical mode propagating through a $\mathrm{Si}_{3} \mathrm{~N}_{4}$ waveguide core embedded in a $\mathrm{SiO}_{2}$ cladding. The geometry considered consists of a standard single-stripe optical waveguide with core dimensions of $4.4 \mu \mathrm{m} \times 30 \mathrm{~nm}$ centered in a $16 \mu \mathrm{m}$-thick cladding. A so-called interdigitized transducer (IDT) excites the SAW in a piezo-electric lead zirconate titanate (PZT) film located on top of the cladding at frequencies in the order of $90 \mathrm{MHz}$.

We found that a combination of IDT electrodes at the $\mathrm{SiO}_{2}-\mathrm{PZT}$ interface with a floating-potential conducting film at the PZT-air interface would be the most efficient in generating strain in the area where the optical mode is. In this case, the maximum induced strain was obtained for an IDT periodicity $\Lambda=30 \mu \mathrm{m}$ with a PZT thickness $d=4 \mu \mathrm{m}$, resulting in a resonant acoustic frequency of around $90 \mathrm{MHz}$. For a guided fundamental optical mode, having a wavelength of $\lambda=840 \mathrm{~nm}$ and a quasi-TM polarization, the strain generated via SAWs was determined to provide a maximum relative change in effective refractive index for the fundamental mode of $\Delta n / n_{\text {eff }}=0.14 \%$, when the IDT is driven with a $10 \mathrm{~V}$ amplitude and at a frequency of $90 \mathrm{MHz}$. This modulation frequency is four orders of magnitude higher than current thermal methods and two orders of magnitude higher than other strain-based 
approaches [6]. Using the maximum change in effective refractive index, full contrast amplitude modulation can be obtained in a Mach-Zehnder interferometer with an arm length as short as $103 \mu \mathrm{m}$ if the two arms of the interferometer are separated by half an acoustic wavelength. This length is about an order of magnitude shorter than the length of typical thermal amplitude modulators for this platform and two orders of magnitude shorter than the strain-based method investigated by Hosseini et al. [6].

Our modeling suggests that the strength of the acousto-optic interaction for phaseand amplitude modulation in stoichiometric silicon nitride waveguides can be further optimized by reducing the distance between the PZT layer and waveguide core. Other methods include focusing of SAW waves [7] or considering other waveguide devices (e.g., ring resonators or multi-mode interferometers). A further advantage of using a SAW to drive the phase- or amplitude modulator appears promising for future realization, which is that a single SAW may be used to coherently drive multiple modulators.

Having on-chip phase- and amplitude modulators for the stoichiometric silicon nitride waveguide platform will benefit many applications. Here, we want to highlight one such application, an on-chip optical isolator for narrow-band light sources. This optical isolator would make use of two phase modulators that are driven 90 degrees out of phase and are separated by a distance of $v_{g} / 4 f_{m}$ [8], where $v_{g}$ is the group velocity of the wave and $f_{m}$ is the modulation frequency of the phase modulators. Using SAW-driven phase modulators, the modulation frequency of the order of $100 \mathrm{MHz}$ may, on first view, be a disadvantage, because a large distance between the modulators would be required. This would be associated with huge optical losses in standard semiconductor waveguides. In standard dielectric waveguides, e.g., fabricated from doped silica, the losses would be low, but, due to the low index contrast of silica, high bending losses would inhibit realization of a chip. The present $\mathrm{Si}_{3} \mathrm{~N}_{4}$ platform, however, offers both low losses and a high index contrast, which warrants the realization of a low-loss acoustic isolator much higher chances.

In Chapter 5 we report for the first time the observation of second-harmonic generation in LPCVD-grown stoichiometric $\mathrm{Si}_{3} \mathrm{~N}_{4}$ waveguides. The output of a modelocked Yb-fiber laser, producing 1064-nm optical pulses with a duration of 6.2 ps at a $20 \mathrm{MHz}$ repetition rate, was frequency doubled in various waveguides with different core dimensions. Second-harmonic generation was only observed after a build-up time in the order of several minutes up to hours, depending on the average IR pump power and waveguide core cross-section. Interestingly, phase matching was found fulfilled independent of the waveguide dispersion. These findings, and the excellent agreement with specific exponential growth rates, are consistent with the coherent photogalvanic effect being responsible for building up of an effective second-order nonlinearity. This effective nonlinearity has a spatial periodicity that 
results in quasi-phase matching for second-harmonic generation. With the available pump laser pulses, the investigated $\mathrm{Si}_{3} \mathrm{~N}_{4}$ waveguides provide a maximum conversion efficiency of $0.4 \%$ for an input power of $13 \mathrm{~mW}$ (corresponding to a peak input power of $105 \mathrm{~W}$ and an input pulse energy of $0.65 \mathrm{~nJ}$ ). To our knowledge, this is the highest conversion efficiency achieved in an integrated SiN waveguide. Assuming that the second-harmonic output is dominantly produced in the transverse mode $E_{13, x}$, such conversion efficiency corresponds to an effective $\chi^{(2)}$ of $3.7 \pm 0.2 \mathrm{pm} / \mathrm{V}$. This value is slightly smaller than that from silicon-rich SiN films [9], measured via X-ray photoelectron spectroscopy, and larger than that achieved with periodic gratings in thin SiN films [10] and SiN ring resonators [11], measured via secondharmonic generation.

The presence of an effective second-order susceptibility in amorphous stoichiometric $\mathrm{Si}_{3} \mathrm{~N}_{4}$ waveguides is important because it may open up various second-order nonlinear optical applications, e.g., generation of entangled photon pairs via parametric down conversion for integrated quantum photonics [12] or optical parametric oscillators [13, 14]. Another application would be a compact, single-chip stabilization of femtosecond laser sources and frequency combs through self-referencing [15, 16]. Here, the third-order nonlinearity of the waveguide would be used for wider than octave-spanning supercontinuum generation, or Kerr frequency comb generation [17], and the effective second-order nonlinearity might be used to implement the $f-2 f$ self-referencing scheme [15] on the same chip. Such sources are of high interest for a broad range of applications, e.g., ultra-high speed communication in interconnects [18], free-space communication [19], coherent telecommunication techniques [20] and precision metrology [21]. Locking one of the frequency components to the atomic clock standard would accommodate on-chip or portable atomic clocks for navigation services [22].

The results presented in this thesis show that nonlinear properties of stoichiometric silicon nitride waveguides can make important contributions to the optical functionality of this waveguide platform. As the technology is compatible with CMOS fabrication, this platform benefits from a mature CMOS manufacturing base and we foresee a bright future with the platform providing economically viable integrated photonic solutions for industry and society.

\section{References}

1. Epping, J. P. et al. On-chip visible-to-infrared supercontinuum generation with more than $495 \mathrm{THz}$ spectral bandwidth. Opt. Express 23, 19596-19604 (2015).

2. Levy, J. Integrated Nonlinear Optics In Silicon Nitride Waveguides And Resonators PhD thesis (2011). 
3. Ikeda, K., Saperstein, R. E., Alic, N. \& Fainman, Y. Thermal and Kerr nonlinear properties of plasma-deposited silicon nitride/ silicon dioxide waveguides. Opt. Express 16, 12987-12994 (2008).

4. Epping, J. P., Kues, M., van der Slot, P. J. M., Lee, C. J., Fallnich, C. \& Boller, K.-J. Integrated CARS source based on seeded four-wave mixing in silicon nitride. Opt. Express 21, 32123-32129 (2013).

5. Nguyen, V. D., Weiss, N., Beeker, W., Hoekman, M., Leinse, A., Heideman, R. G., van Leeuwen, T. G. \& Kalkman, J. Integrated-optics-based swept-source optical coherence tomography. Opt. Lett. 37, 4820-4822 (2012).

6. Hosseini, N., Dekker, R., Hoekman, M., Dekkers, M., Bos, J., Leinse, A. \& Heideman, R. Stress-optic modulator in TriPleX platform using a piezoelectric lead zirconate titanate (PZT) thin film. Opt. Express 23, 14018-14026 (2015).

7. Collins, D. J., Neild, A. \& Ai, Y. Highly focused high-frequency travelling surface acoustic waves (SAW) for rapid particle sorting. Lab. Chip 16, 471 (2016).

8. Doerr, C. R., Dupuis, N. \& Zhang, L. Optical isolator using two tandem phase modulators. Opt. Lett. 36, 4293-4295 (2011).

9. Kitao, A., Imakita, K., Kawamura, I. \& Fujii, M. An investigation into second harmonic generation by Si-rich SiN thin films deposited by RF sputtering over a wide range of Si concentrations. J. Phys. D: Appl. Phys. 47, 215101 (2014).

10. Ning, T., Pietarinen, H., Hyvärinen, O., Kumar, R., Kaplas, T., Kauranen, M. \& Genty, G. Efficient second-harmonic generation in silicon nitride resonant waveguide gratings. Opt. Lett. 37, 4269-4271 (2012).

11. Levy, J. S. \& Lipson, M. Harmonic generation in silicon nitride ring resonators. Opt. Express 17, 11366-11370 (2011).

12. Eisaman, M. D., Fan, J., Migdall, A. \& Polyakov, S. V. Invited Review Article: Singlephoton sources and detectors. Rev. Sci. Intrum. 82, 071101 (2011).

13. Boyd, R. W. Nonlinear Optics (Academic Press, 2013).

14. Myers, L. E., Eckardt, R. C., Fejer, M. M., Byer, R. L., Bosenberg, W. R. \& Pierce, J. W. Quasi-phase-matched optical parametric oscillators in bulk periodically poled $\mathrm{LiNbO}_{3}$. J. Opt. Soc. Am. B 12, 2102-2116 (1995).

15. Jones, D. J., Diddams, S. A., Ranka, J. K., Stentz, A., Windeler, R. S., Hall, J. L. \& Cundiff, S. T. Carrier-Envelope Phase Control of Femtosecond Mode-Locked Lasers and Direct Optical Frequency Synthesis. Science 288, 635-639 (2000).

16. Carlson, D. R. et al. Self-referenced frequency combs using high-efficiency silicon-nitride waveguides. Opt. Lett. 42, 2314 (2017).

17. Okawachi, Y., Saha, K., Levy, J. S., Wen, Y. H., Lipson, M. \& Gaeta, A. L. Octavespanning frequency comb generation in a silicon nitride chip. Optics Letters 36, 3398 $(1,2011)$.

18. Levy, J. S., Gondarenko, A., Foster, M. A., Turner-Foster, A. C., Gaeta, A. L. \& Lipson, M. CMOS-compatible multiple-wavelength oscillator for on-chip optical interconnects. Nat. Photon. 4, 37-40 (2010).

19. Nagatsuma, T., Ducournau, G. \& Renaud, C. C. Advances in terahertz communications accelerated by photonics. Nat. Photon. 10, 371-379 (2016).

20. Pfeifle, J. et al. Coherent terabit communications with microresonator Kerr frequency combs. Nat. Photon. 8, 375-380 (2014). 
21. Coddington, I., Swann, W. C., Nenadovic, L. \& Newbury, N. R. Rapid and precise absolute distance measurements at long range. Nat. Photon. 3, 351-356 (2009).

22. Del'Haye, P., Coillet, A., Fortier, T., Beha, K., Cole, D. C., Yang, K. Y., Lee, H., Vahala, K. J., Papp, S. B. \& Diddams, S. A. Phase-coherent microwave-to-optical link with a self-referenced microcomb. Nat. Photon. 10, 516-520 (2016). 


\section{Publication List}

\section{Journals}

[1] Marco A.G. Porcel, Florian Schepers, Jörn P. Epping, Tim Hellwig, Marcel Hoekman, René G. Heideman, Peter J. M. van der Slot, Chris J. Lee, Robert Schmidt, Rudolf Bratschitsch, Carsten Fallnich, and Klaus-J. Boller, "Twooctave spanning supercontinuum generation in stoichiometric silicon nitride waveguides pumped at telecom wavelengths," Opt. Express, vol. 25, pp. 15421554, (2017).

[2] Marco A.G. Porcel, Jesse Mak, Caterina Taballione, Victoria K. Schermerhorn, Jörn P. Epping, Peter J. M. van der Slot, Klaus-J. Boller, "Photoinduced $\chi^{(2)}$ for second harmonic generation in stoichiometric silicon nitride waveguides," Proc. SPIE 10228, Nonlinear Optics and Applications X, 102280R (2017).

[3] Marco A. G. Porcel, Remco Stoffer, Matthijn Dekkers, Marcel Hoekman, Ronald Dekker, Arne Leinse, Peter J. M. van der Slot, Klaus-J. Boller, "Surface acoustic waves for acousto-optic modulation in buried silicon nitride waveguides," ArXiv., 1710.03055 (2017).

[2] Marco A.G. Porcel, Jesse Mak, Caterina Taballione, Peter J. M. van der Slot, Klaus-J. Boller, "Photo-induced second-order nonlinearity in stoichiometric silicon nitride waveguides," ArXiv., 1710.03010 (2017). 


\section{Conferences}

[1] M.A.G. Porcel, M. Hoekman, M. Dekkers, R. Stoffer, R. Dekker, A. Leinse, P.J.M. van der Slot, K.-J. Boller," Enabling acousto-optic devices for glass waveguide, "IEEE Photonics Benelux Chapter 2014, 157-160, Eindhoven, The Netherlands (2014).

[2] M.A.G. Porcel, M. Hoekman, M. Dekkers, R. Stoffer, R. Dekker, A. Leinse, P.J.M. van der Slot, K.-J. Boller," High speed modulators in buried silicon nitride waveguides, " EOSAM2014, Berlin, Germany (2014).

[3] M.A.G. Porcel, M. Hoekman, M. Dekkers, R. Stoffer, R. Dekker, A. Leinse, P.J.M. van der Slot, K.-J. Boller, " $\mathrm{MHz}$ acousto-optics modulation in glass waveguides via integrated Mach-Zehnder interferometer, "Summer School New Frontiers in Optical Technologies, Tampere, Finland (2015).

[4] F. Schepers,M.A.G. Porcel, J. P. Epping, T. Hellwig, M. Hoekman, R. Mateman, A. Leinse, R. G. Heideman, A. van Rees, P. J. M. van der Slot, C. J. Lee, R. Schmidt, R. Bratschitsch, K. Boller, and C. Fallnich, "Ultra-broadband Supercontinuum Generation at Telecommunication Wavelengths in Dispersion Engineered Stoichiometric $\mathrm{Si}_{3} \mathrm{~N}_{4}$ Waveguides," in Conference on Lasers and Electro-Optics, San Jose, CA, USA, (2016), paper AM3J.5.

[5] M.A.G. Porcel, J. P. Epping, M. Hoekman, P.J.M. van der Slot, K.-J. Boller, "Second-harmonic generation in stoichiometric silicon nitride glass waveguides, " in Conference on Lasers and Electro-Optics - European Quantum Electronics Conference, Munich, Germany (2017). 


\section{Acknowledgements}

This thesis is the result of the influence, work and passion of many people. Some of them will not be recognized, be aware of it or, even, understand what has been accomplished. Fortunately, some others will. I want to thank all of you for what you have helped accomplish, up to the last one of you. As for every story, there is a beginning. I will take the cliche and start by thanking my parents, who are probably as guilty of this work as my promoter and co-promoter. Pushing me as a young lad to continue studying and not punishing me when I was reading books instead of doing homework. To my sisters, from whom I learn more than they can imagine. And to my grandparents from whom I learned to appreciate nature, love food and respect everyone. Since a continuous stream of names and thoughts, although comforting, would take too much, I am going to cut to the meat.

In my time in Enschede I met a lot of people, from them there are several that have suffered me during my best and worst days. Chris, Mark, Laura, Rob, Kay, Karen, Matthijs and later Franz and Nat, you guys taught me how to not fall from the bike so often, and withstand me every time I complained about all sort of things. We shared sunny evenings and cold mornings between beers and coffee always in great fun. I am keeping you with me, wherever I go. Thank you guys. 3,2,1, POLO. Chris, as you know everyone needs fake doors and diamons in the sky.

A very interesting feature of Dutch universities are their student sport clubs. Among these, I will always remember the Twente Student Climbing Association. You guys have a great association there. Maikel, Jurrian, keep it up and thanks for all the fish.

Within the people I met during my time as PhD student at the University of Twente, there are two incredibly smart and talented persons that I had the luck to meet, one of them was my first flatmate in Enschede, Guillaume, and a friend that he introduced me to, Rodolfo, who would later introduce me to my wife. Thanks guys for your unconditional friendship, your humor, the late night physics and philosophy discussions, the smelly cheese, the wine and the tons of laughter.

On the academic side of things (not all could be sports and wine), I met many colleagues at the ANP or Applied NanoPhotonics initiative which encompasses all the optical groups at the University of Twente. From these chats, I was inspired many times. Often out of my own research, but, isn't that what is all about. 
As often happens with nowadays research, it could not have been realized without the contributions of industrial partners. I have been very fortunate to have been able to work with several different industrial partners during my research. I want to give special thanks to Arne, Ronald, and Marcel from LioniX Int., Matthijn from Solmates, and Remco from PhoeniX, without whom this research would not have been possible.

With special regard, I want to thank all the people at the Optical Technologies group in the Universität Münster lead by prof. $d r$. C. Fallnich for their warm welcome and their hospitality, expecially, during the time Florian and I were performing the supercontinuum experiments. Thanks guys for speaking English during the lunch breaks, Essen!

Last but not certainly not least, I want to thank all the people at the Laser Physics and Nonlinear Optics group at the University of Twente. Yin, yes indeed haha means fun, there has never been a better beamblock, sickness warning, do not mix with Kasper. Kasper, our personal plume spectroscopy expert with whom I could always have a fun argument about anything, keep it up with the icecream. Muharem, I have never met someone as chill and easy going as you, really impressive. Jörn, I could have save so much time by being your shadow a few months. Jonathan, I will always remember you as the calm man with an impassible twisted dark humor. Caterin- $a$, you came half way through your Ph.D to our group, a life-changing experience with no doubt, you are next!. Youwen, a.k.a. laserman, and now also Ba' Youwen, your humor and your good words were always refreshing. Hard times with you around in the office or in the lab became a lot easier to overcome. Jesse, the man for all tasks, there is light at the end of the waveguide, that I can say, don't panic. Caterin- $b$, the voice of the office, queen-of-waveguides. Note: never reject live opera in the lab. Tom, a.k.a. handyTom, the man with the inefficient light source that beats records, I am really glad to have met you, I will never forget your last days running experiments an listening to me crying because my experiments where too successful at the last minute and did not know why yet. Peter, my tutor and co-supervisor during this long four and half years. You care for detail, your good eye and impeccable manners are an immense strength to LPNO. This thesis would certainly not have been what it is without your help, your assistance and your corrections. Klaus, my supervisor, I have learned so much from you, in so many aspects of my professional and personal life that I cannot even find a way to start. You have always been very open, welcoming, and correct. I will do my best to follow your example and keep learning without losing what I have got in the time as your student. 


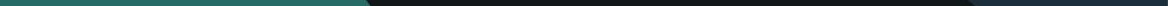

